\title{
User's Manual
}

\author{
J. S. Ishee \\ R. L. Haese \\ R. D. Heatherly \\ S. E. Hughes \\ J. K. Lovin \\ S. M. Pratt \\ D. W. Smith
}


This report has been reproduced directly from the best available copy.

Avallable to DOE and DOE contractors from the Office of Scientific and Technical Information, P.O. Box 62, Oak Ridge, TN 37831; prices available from (615) 576-8401, FTS 626-8401.

Available to the public from the National Technical Information Service, U.S. Department of Commerce, 5285 Port Royal Rd., Springfield, VA 22161.

This report was prepared as an account of work sponsored by an agency of the United States Government. Nelther the United States Government nor any agency thereof, nor any of their employees, makes any warranty, express or implied, or assumes any legal liability or responsibility for the accuracy, completeness, or usefulness of any information, apparatus, product, or process disclosed, or represents that its use would not infringe privately owned rights. Reference herein to any specific commercial product, process, or service by trade name, trademark, manufacturer, or otherwise, does not necessarily constitute or imply its endorsement, recommendation, or favoring by the United States Government or any agency thereof. The views and opinions of authors expressed herein do not necessarily state or reflect those of the United States Government or any agency thereof. 
Finance and Business Management Division

\title{
ORNL ALPHAMA MIS USER'S MANUAL
}

\author{
J. S. Ishee* \\ R. L. Haese* \\ R. D. Heatherly* \\ S. E. Hughest \\ J. K. Lovin \\ S. M. Pratt\$ \\ D. W. Smith*
}

Date Published-February 1992

*Computing and Telecommunications Division.

†Publications Division.

¥Business Systems Division (ALPHA Project Manager).

SFinance and Business Management Division.

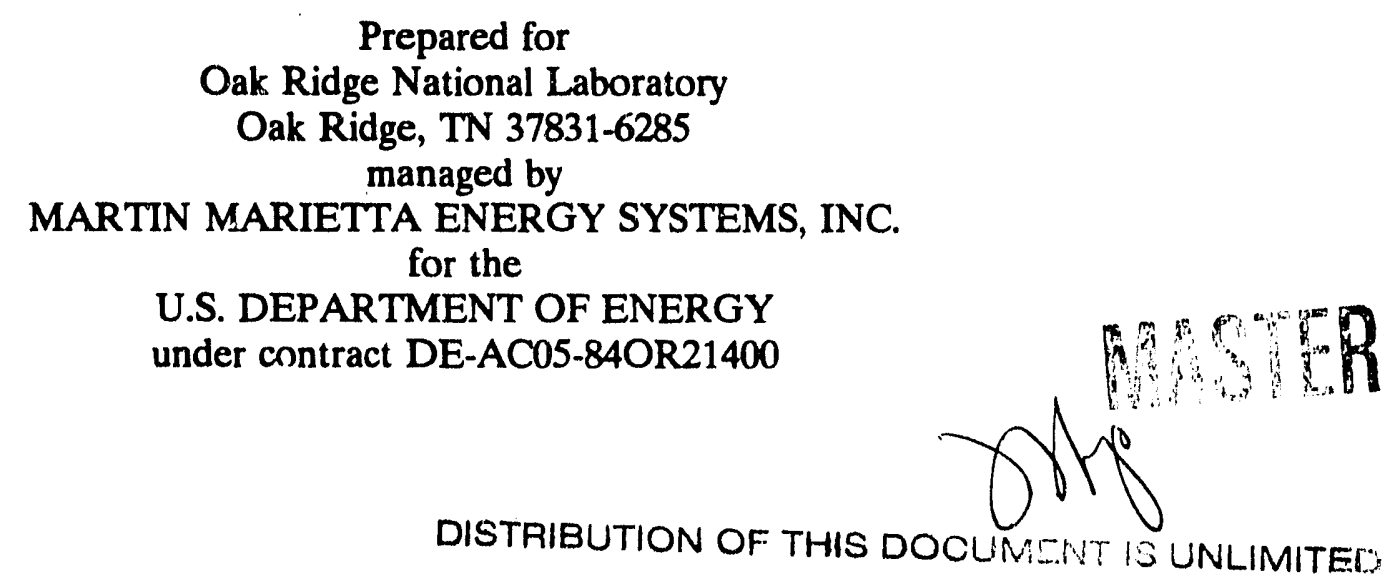


This page intentionally left blank. 
IIST OF TABLES AND EXHIBITS $\ldots \ldots \ldots \ldots \ldots \ldots \ldots \ldots \ldots \ldots \ldots$

PREFACE $\ldots \ldots \ldots \ldots \ldots \ldots \ldots \ldots \ldots \ldots \ldots \ldots \ldots \ldots \ldots \ldots \ldots \ldots$

ACKNOWLEDGMENTS $\ldots \ldots \ldots \ldots \ldots \ldots \ldots \ldots \ldots \ldots \ldots \ldots \ldots \ldots \ldots$

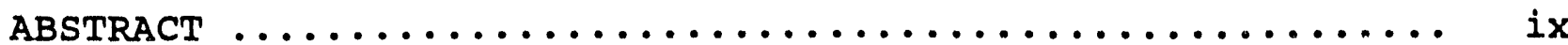

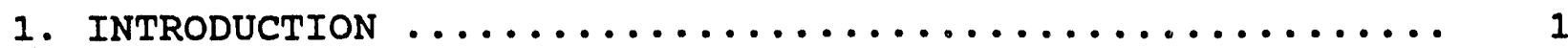

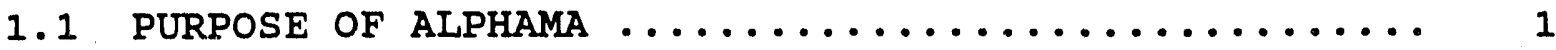

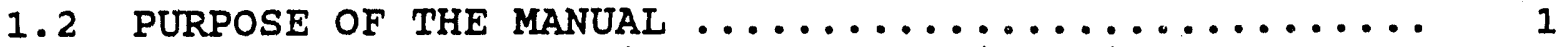

1.2.1 Example Sessions and Practice Files ........ 2

1.2.2 Typographic Conventions on Documentation ... 2

2. INPUT BASICS: COMMANDS AND ATTRIBUTES .............. 3

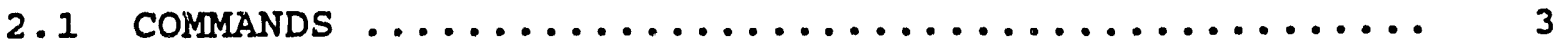

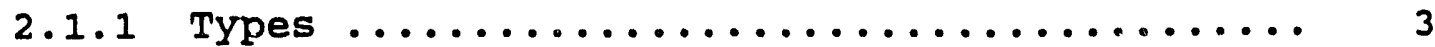

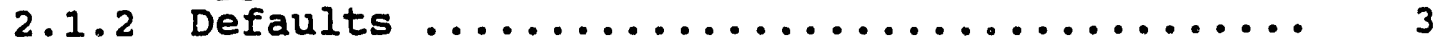

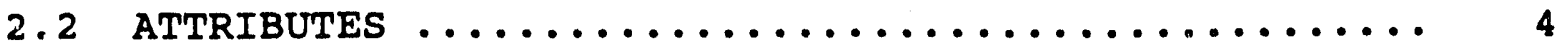

2.2 .1 Types $\ldots \ldots \ldots \ldots \ldots \ldots \ldots \ldots \ldots \ldots \ldots \ldots \ldots \ldots \ldots \ldots$

2.2.1.1 Text: Short and Long ........... 4

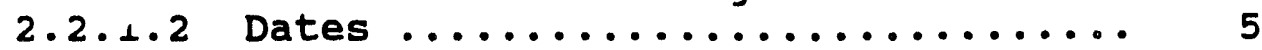

2.2 .1 .3 Integers ................... 6

2.2.1.4 Real Numbers: Decimal $\ldots \ldots \ldots \ldots \ldots$

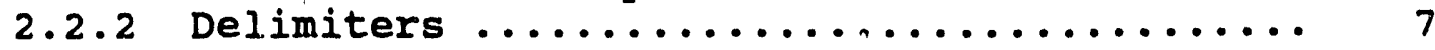

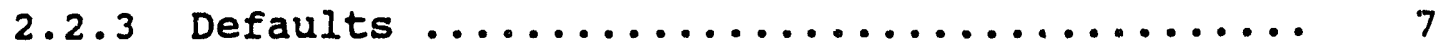

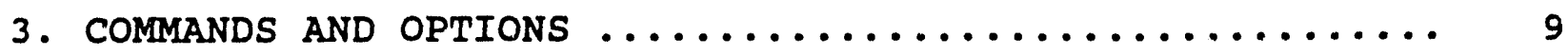

3.1 ACCESSING THE PROGRAM .................... 9

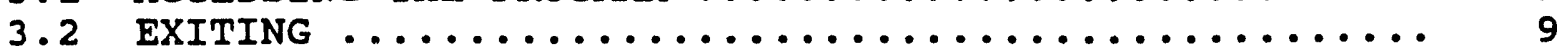

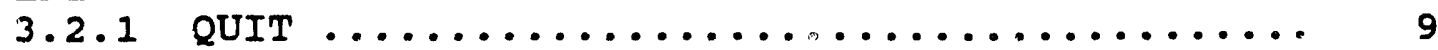

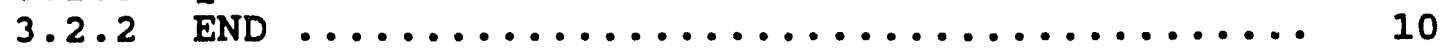

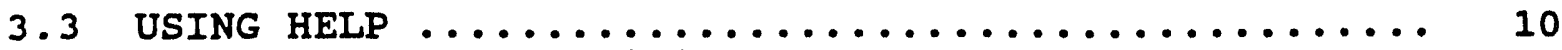

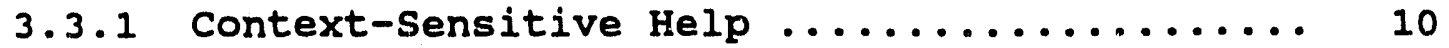

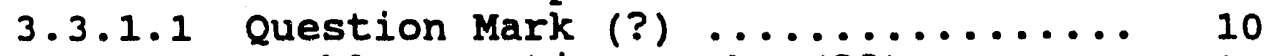

3.3.1.2 Double Question Marks (??) ........ 11

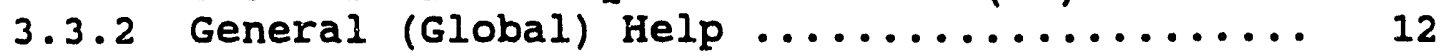

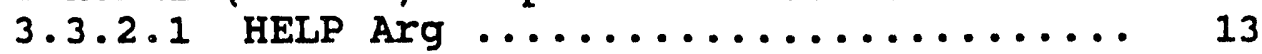

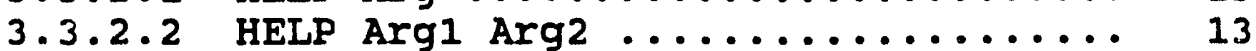

3.4 OPENING A DATABASE: DATABASE COMMAND ............ 14

3.5 SELECTING RECORDS: SEARCH MODE .............. 16 
3.5.1 Specification and Retrieval (UID) ........ 16

3.5 .2 Help $\ldots \ldots \ldots \ldots \ldots \ldots \ldots \ldots \ldots \ldots \ldots \ldots \ldots \ldots \ldots \ldots \ldots \ldots, 18$

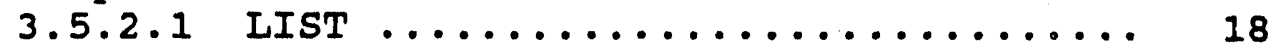

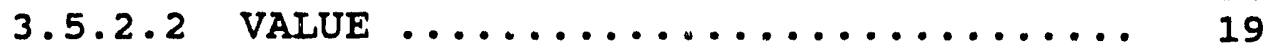

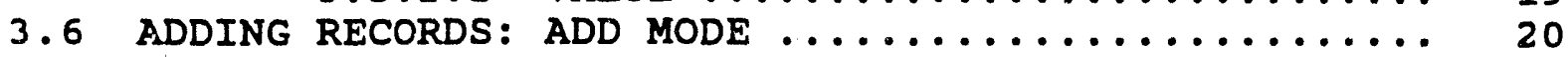

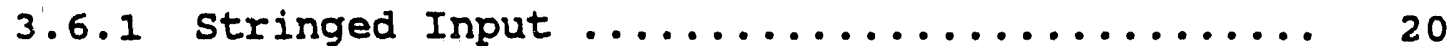

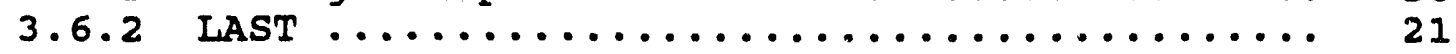

3.6 .3 SKIP and SKIP Att $\ldots \ldots \ldots \ldots \ldots \ldots \ldots \ldots, 21$

3.6 .4 Auto Update $\ldots \ldots \ldots \ldots \ldots \ldots \ldots \ldots \ldots \ldots, 22$

3.6 .5 Star (*) Multiplier ................. 23

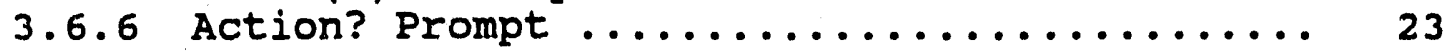

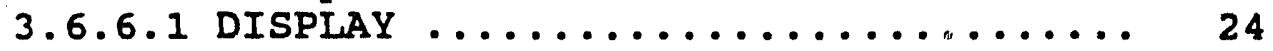

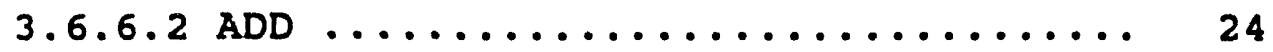

3.6 .6 .3 EDIT $\ldots \ldots \ldots \ldots \ldots \ldots \ldots \ldots \ldots \ldots \ldots \ldots 24$

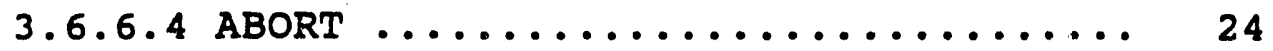

3.6 .6 .5 END and <Enter> $\ldots \ldots \ldots \ldots \ldots \ldots .24$

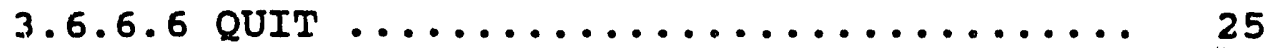

3.7 EDITING RECORDS: EDIT MODE $\ldots \ldots \ldots \ldots \ldots \ldots \ldots \ldots, 25$

3.7.1 Short Text Attributes ................ 25

3.7 .2 Long Text Attributes $\ldots \ldots \ldots \ldots \ldots \ldots \ldots, 25$

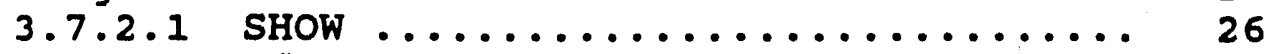

$3.7 .2 .2<\#>$ (Delete value) $\ldots \ldots \ldots \ldots \ldots 26$

3.7.2.3 <Enter> (Display Next Line) ...... 26

$3.7 .2 .4\langle\wedge$ (Display Previous Line) ...... 26

$3.7 .2 .5<D>$ (Delete Line) ............. 27

$3.7 .2 .6<$ I $>$ (Insert) $\ldots \ldots \ldots \ldots \ldots \ldots \ldots, 27$

$3.7 .2 .7<A>$ (Append) $\ldots \ldots \ldots \ldots \ldots \ldots \ldots 27$

$3.7 .2 .8<R>$ (Replace String) $\ldots \ldots \ldots \ldots \ldots 27$

3.7 .2 .9 END $\ldots \ldots \ldots \ldots \ldots \ldots \ldots \ldots \ldots \ldots \ldots, 28$

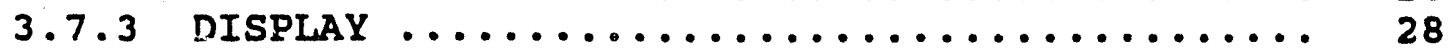

3.7 .4 Att $\ldots \ldots \ldots \ldots \ldots \ldots \ldots \ldots \ldots \ldots \ldots \ldots \ldots \ldots \ldots \ldots \ldots \ldots, 28$

3.7 .5 SKIP Att $\ldots \ldots \ldots \ldots \ldots \ldots \ldots \ldots \ldots \ldots \ldots \ldots \ldots \ldots \ldots \ldots \ldots, 28$

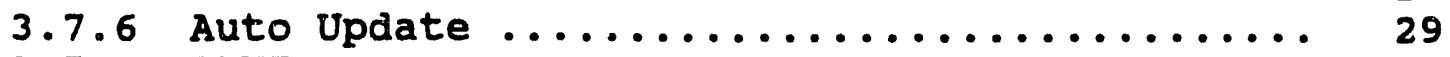

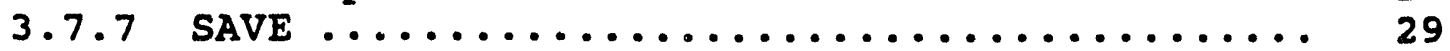

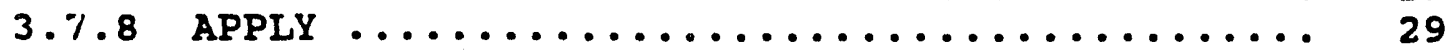

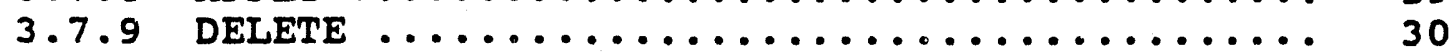

3.7 .10 MOVE $(P D P-10) \ldots \ldots \ldots \ldots \ldots \ldots \ldots \ldots \ldots \ldots \ldots \ldots, 31$

3.7 .11 ABORT $\ldots \ldots \ldots \ldots \ldots \ldots \ldots \ldots \ldots \ldots \ldots \ldots \ldots \ldots \ldots, 32$

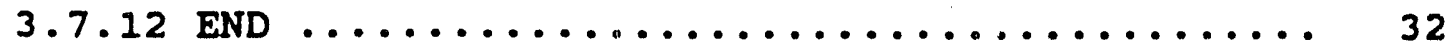

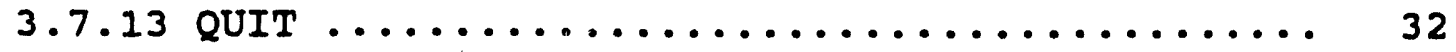

4. SUPPORT AND AUXILIARY FILES $\ldots \ldots \ldots \ldots \ldots \ldots \ldots \ldots \ldots, 33$

4.1 DATA DESCRIPTION (DBNAME. DMD) $\ldots \ldots \ldots \ldots \ldots \ldots \ldots, 33$

4.2 MASTER TABLE (DBNAME. TBL) $\ldots \ldots \ldots \ldots \ldots \ldots \ldots \ldots \ldots, 34$

4.3 REFERENCED DATA (DBNAME.REF) $\ldots \ldots \ldots \ldots \ldots \ldots \ldots \ldots, 37$

4.3.1 Referenced Attributes ............... 38

4.3 .2 Coded Referenced Attributes ........... 38

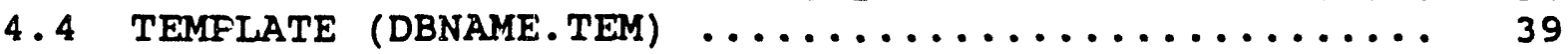

4.5 CHANGE LOG (DBNAME. CHA) $\ldots \ldots \ldots \ldots \ldots \ldots \ldots \ldots \ldots \ldots \ldots \ldots \ldots, 40$ 


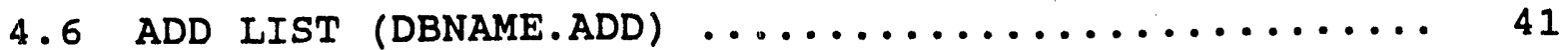

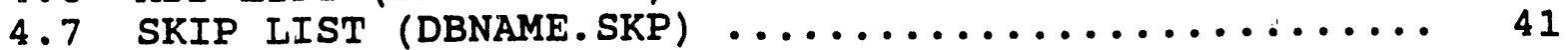

4.8 MOVED PDP-10 RECORDS (DBNAME.MOV) ............ 41

4.9 ERROR FILE (DBNAME.ERR) $\ldots \ldots \ldots \ldots \ldots \ldots \ldots \ldots \ldots \ldots \ldots \ldots . \ldots \ldots$

5. $\mathrm{KSV}$ VAX EXAMPLE SESSIONS ................... 43

5.1 SESSIJN 1: ADD A RECORD .................. 45

5.2 SESSION 2: EDIT A RECORD ................... 54

5.3 SESSION 3: DELETE A RECORD ................... 63

6. REFERENCES .................................. 65

APPENDIX A. COMMANDS: LOGICAL VIEW OF A TYPICAL SESSION .... 67

A. 1 GENERAL COMMANDS AND OPTIONS .............. 67

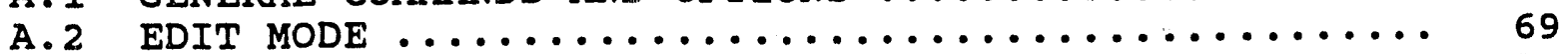

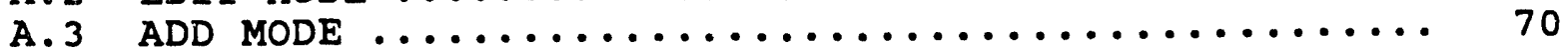

APPENDIX B. KSV VAX EXAMPLE SUPPORT AND AUXILIARY FILES ... 73

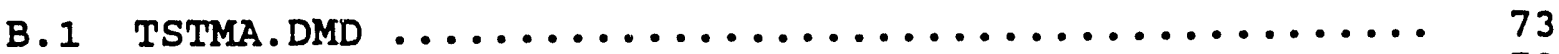

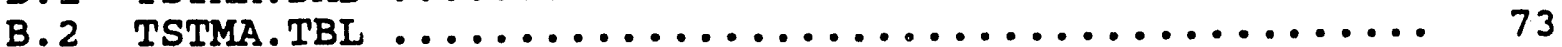

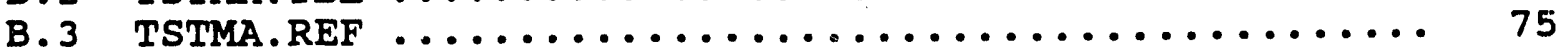

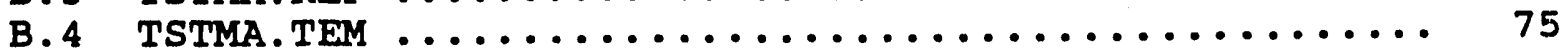

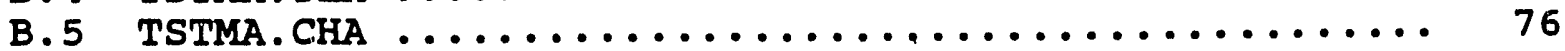

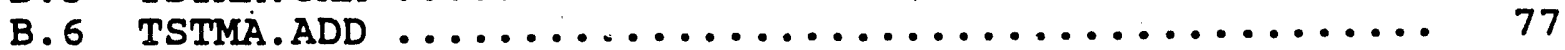

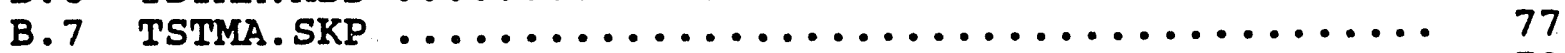

B. 8 TSTMA.ERR $\ldots \ldots \ldots \ldots \ldots \ldots \ldots \ldots \ldots \ldots \ldots \ldots \ldots \ldots \ldots \ldots$

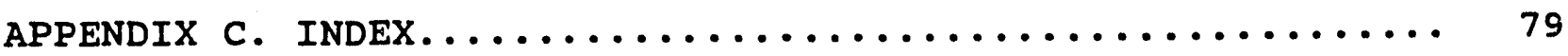

READER COMMENT FORM .......................... 87 


\section{I 8 T O F T A B I E 8 A N D E X H I B I T 8}

T A B L E S

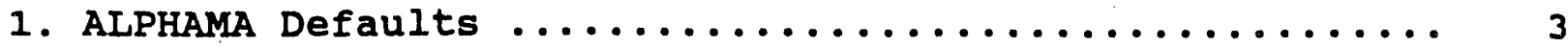

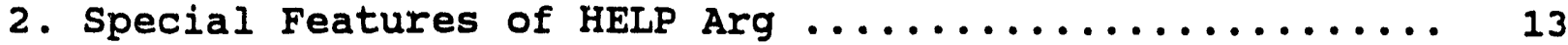

3. Options at the ALPHAMA Add? Prompt .............. 20

4. Structure section of the Data Description

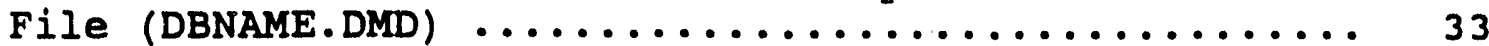

5. Contents of the Master Table (DBNAME.TBL) .......... 33

6. Format of the Change Log file (DBNAME. CHA) ......... 40

7. Data for the Example New Record ................. 45

8. Updated Attribute Values of the Example Edited Record ............................ 54

A.1. General Command options .................... 67

A.2. Commands and options in SEARCH Mode ............. 68

A.3. Commands and options in EDIT Mode When

a Record has Been Found ..................... 69

A.4. Commands and Options in SEARCH Mode When

a Record Has Not Been Found .................. 70

A.5. Commands and Options in ADD Mode at the Attribute Prompts for a New Record .................... 71

A.6. Commands and options in ADD Mode at the Action? Prompt To Process a New Record ................ 72

E X H I B I T $\mathbf{S}$

1. Example of a Comma Used as Delimiter in ADD Mode ..... 7

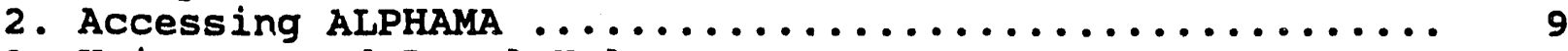

3. Using Second-Level Help ...................... 11

4. Using Third-Level Help at the <Database> Prompt ...... 12

5. Using Third-Level Help at the Unique

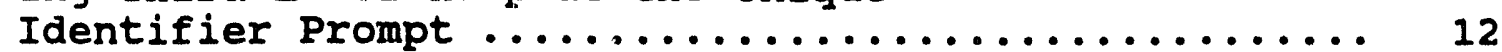

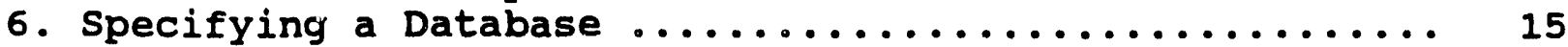

7. Selecting Records with Unique Identifiers .......... 16

8. Deleting Duplicate Records .................. 17

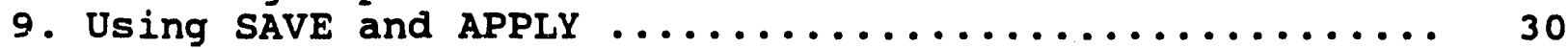

10. Deleting a Record ......................... 30

11. Using MOVE To Delete a Record
on the PDP-10 Computer $\ldots \ldots \ldots \ldots \ldots \ldots \ldots \ldots \ldots \ldots \ldots \ldots \ldots \ldots \ldots \ldots$

B.1. Data Description File TSTMA.DMD ................ 73

B.2. Master Table TSTMA.TBL ................... 74

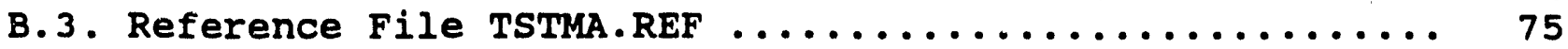

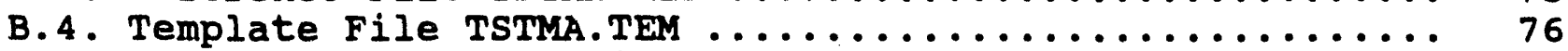

B.5. Example Change Log File TSTMA.CHA ............. 76

B.6. Add File TSTMA.ADD ...................... 77

B.7. Skip File TSTMA.SKP $\ldots \ldots \ldots \ldots \ldots \ldots \ldots \ldots \ldots \ldots \ldots \ldots \ldots$

B.8. Example Error File TSTMA.ERR $\ldots \ldots \ldots \ldots \ldots \ldots \ldots \ldots \ldots$ 
This manual is one in a series of manuals documenting the management information system (MIS) ALPHA, developed at Martin Marietta Energy systems, Inc. To ensure that all users have immediate access to reference documentation, its manuals are stored online as flat (ASCII) files; users can simply output them to their own printer. We feel that the advantages of such accessibility outweigh the typographic limitations imposed by the ASCII format.

The series includes the following manuals (which are available from within ALPHA (using the Documentation command) or at the system prompt (using the Type command)] :

* ORNL ALPHA MIS User's Manual (TM-8621) -- A manual that documents ALPHA's commands and options

* ORNL ALPHAMA MIS User's Manual (TM-8622) -- A manual for the person responsible for maintaining data that documents how ALPHAMA's commands and options work

* ORNL ALPHA MIS Programmer's Manual (TM-8623) and ORNL ALPHAMA MIS Programmer's Manual (TM-8923) -- Manuals for programmers that document ALPHAMA software (not available to general users)

* ORNL ALPHA MIS Data Base Manual (TM-8655) -- A manual that explains how to design and set up both a database and support files utilized by ALPHA

The ALPHA system developers value and appreciate your comments as an ALPHA System user and reader of the manuals. As we write, revise, and evaluate, your opinions continue to be the most important input we receive. At the back of this manual is a Reader's comment Form that we encourage you to use to tell us what you both like and dislike about this (or any other) ALPHA system manual or product. If the form is gone or you would like to contact us, please write to us at Jackson Plaza, MS-7610 in care of the Business Systems Information Center, or call us at 6-2859.

J. K. Lovin Project Manager 
This manual and the work it documents are the result of the cumulative efforts of several individuals who deserve recognition for their contributions, especially J. K. Lovin (Business Systems Division), project manager. Thanks go to other ALPHA team programmers (Computing and Telecommunications Division) for their review and comments and to $K$. D. Warden (Finance and Business Management Division) for program testing. The second edition is clearly grounded in the work of the authors of the first edition: project manager $J$. K. Lovin, contract monitor R. I. Haese (Computing and Telecommunications Division), P. L. Bowen (Finance and Business Management Division), and J. W. Grubb (Planning Research Corporation). Special thanks are due the contributors to the first edition: $R$. K. Davis (Planning Research Corporation and subsequently Computing and Telecommunications Division) for software development; J. G. Lovelace (Finance and Business Management Division) for software testing; and T. G. Yow (Publications Division) for documentation coordination. 
ALPHAMA is a command-driven application that allows Martin Marietta Energy systems, Inc., employees to maintain any system 1022 or 1032 database located on its Digital Equipment Corporation, Inc., PDP-10 and VAX computers. Assisted by online Help, users can access a database, specify records of interest, and perform necessary maintenance. With ALPHAMA, they can add records, delete records, and/or change the value of attributes in existing records. This manual presents the various ALPHAMA commands, options, and support files that provide these maintenance functions. It also includes three example sessions as a short introduction to ALPHAMA for the new user. The appendixes provide sample commands and support files 
This page intentionally left blank. 


\subsection{P D R P O B E O F A I P H A M A}

ALPHAMA is command-driven software that allows users to maintain any system 1022 or 1032 database on Digital Equipment Corporation, Inc., PDP-10 and VAX computers. It provides the maintenance function for databases accessible from ALPHA, a general-purpose management information system (MIS) initiated and sponsored by the Finance and Business Management Division of Oak Ridge National Laboratory (ORNL) and developed and maintained in concert with the Business Systems Division for its Information center. ALPHAMA provides users who have the authority to update a database with the capability to

1. add records,

2. change the values of attributes of existing records, and

3. delete records.

ALPHAMA is a base program designed and written for easy modification and/or expansion. Its DBNAME.TBL, DBNAME.REF, DBNAME.ADD, and DBNAME.SKP support files satisfy many, if not ost, maintenance requirements.

When maintenance requirements demand capabilities beyond those provided by ALPHAMA and its support files, however, ALPHA modules provide the capabilities required for creating a custom fintenance program (MA) with ALPHAMA as its base. The DBBUILDER progtw can help users build custom databases and support and auxiliary files. ALPHAMA can provide the basic maintenance functions, and specialized routines (e.g., TFJSMA and SUBMA) can provide the remaining functions. (See Ref. 1 for a discussion of the process of creating a database, master table, and other support files.) For information on a customized MA, contact the Business systems Information Center (Jackson Plaza, MS-7610).

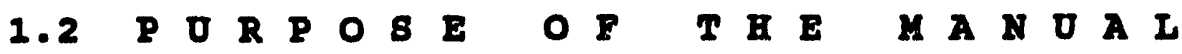

This user manual is one of a suite of aids designed to provide a broad spectrum of program documentation (e.g., Refs. 1 to 4 ) to ALPHA users, from a context-sensitive and global Help system to timely electronic notices (Hotnews) on ALPHAMA updates. It documents version 5C (V5C) of ALPHAMA.

Chapter 2 discusses scue basics about ALPHAMA commands and the database attributes it can manipulate. Chapter 3 provides a detailed explanation of how to use ALPHAMA commands to add, edit, 
and delete data -- primarily on KSV VAX System 61. (Although accessing ALPHAMA is slightly different for a VAX computer user than for a PDP-10 user, once ALPHAMA is running, its commands are the same for both systems.) Chapter 4 is composed of support and auxiliary files. Chapter 5 documents three example maintenance sessions (adding, editing, and deleting records) that users can replicate on a VAX computer.

\subsubsection{Example Bessions and Practice Files}

The example ALPHAMA sessions in Chap. 5 [adding (Sect. 5.1), editing (sect. 5.2), and deleting (sect. 5.3) records] are designed as hands-on exercises. A special test database (TSTMA) and auxiliary files are stored on the KSV VAX computer for ALPHAMA users. Users may copy the example files into their own computer area and work through the commands described in the chapter:

\section{\$ COPY U7:[INF.TESTDB]TSTMA.* [ ]<Enter>}

NOTE: Discussions and examples in Chap. 5 assume that a database has support files and a master table (Chap. 4) linking them together; however, ALPHAMA can process a database that has no master table or other support files.

\subsubsection{Typographic Conventions on Documentation}

To be accessible to as many users as possible, Information Center manuals are stored as ASCII text files on the mainframe computer (e.g., this manual is stored as UO: [RDH.MANUALS] TM8622.DOC). From there users can download the file or print their own copy (page breaks are based on output to a printer with a 10-character-per-inch monospaced font).

As an online file in ASCII text, this manual relies on basic character distinctions to represent typographic conventions that might otherwise be used with a formatted hardcopy document. Filenames, commands, and most user input are presented by uppercase characters. Keyboard ixeys, which appear with an initial uppercase character, are enclosed in arrow brackets. 


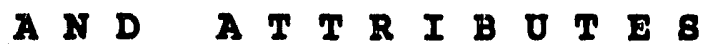

2.1 C O M

\subsubsection{I $y \geq \propto 8$}

ALPHAMA has commands that are valid only at a specific prompt (Sects. 3.4 to 3.7 ) and others that are executable from almost any prompt (Sects. 3.2 and 3.3; Appendix A.1, Sect. A.1). The latter include single and double question marks (? and ??), HELP, END, and QUIT; they provide online help (defining terms, commands, and attributes), transfer control to another part of the program, or exit ALPHAMA. A sixth general option is the <Enter> key; pressing <Enter> initiates the processing of commands, data, or ALPHAMA default.s.

\section{1 .2 D E I u 1 t 8}

ALPHAMA contains a standard system of defaults, that is, responses it processes when the user presses <Enter> instead of typing specific input [e.g., presses <Enter> at a yes or no ( $Y / N$ ?) prompt instead of entering the letter "Y" or "N"]. Defaults peculiar to a specific database are part of its master table, if one exists (sect. 4.2); and when you access a database, ALPHAMA asks if you wish its defaults to be displayed. Table 1 1ists some of the defaults ALPHAMA users can expect.

\section{TABLE 1. ALPHAKA Defaulis}

Feature/Option

Default

Prompt display

[ LONG prompting $(\mathrm{Y} / \mathrm{N})$ ?]

No (short, abbreviated)

YES/NO question

No

Database:

Device (disk)

Directory

Filename

File extension
Disk structure currently being used Directory where user logged on None (user MUST input filename)

DMS 


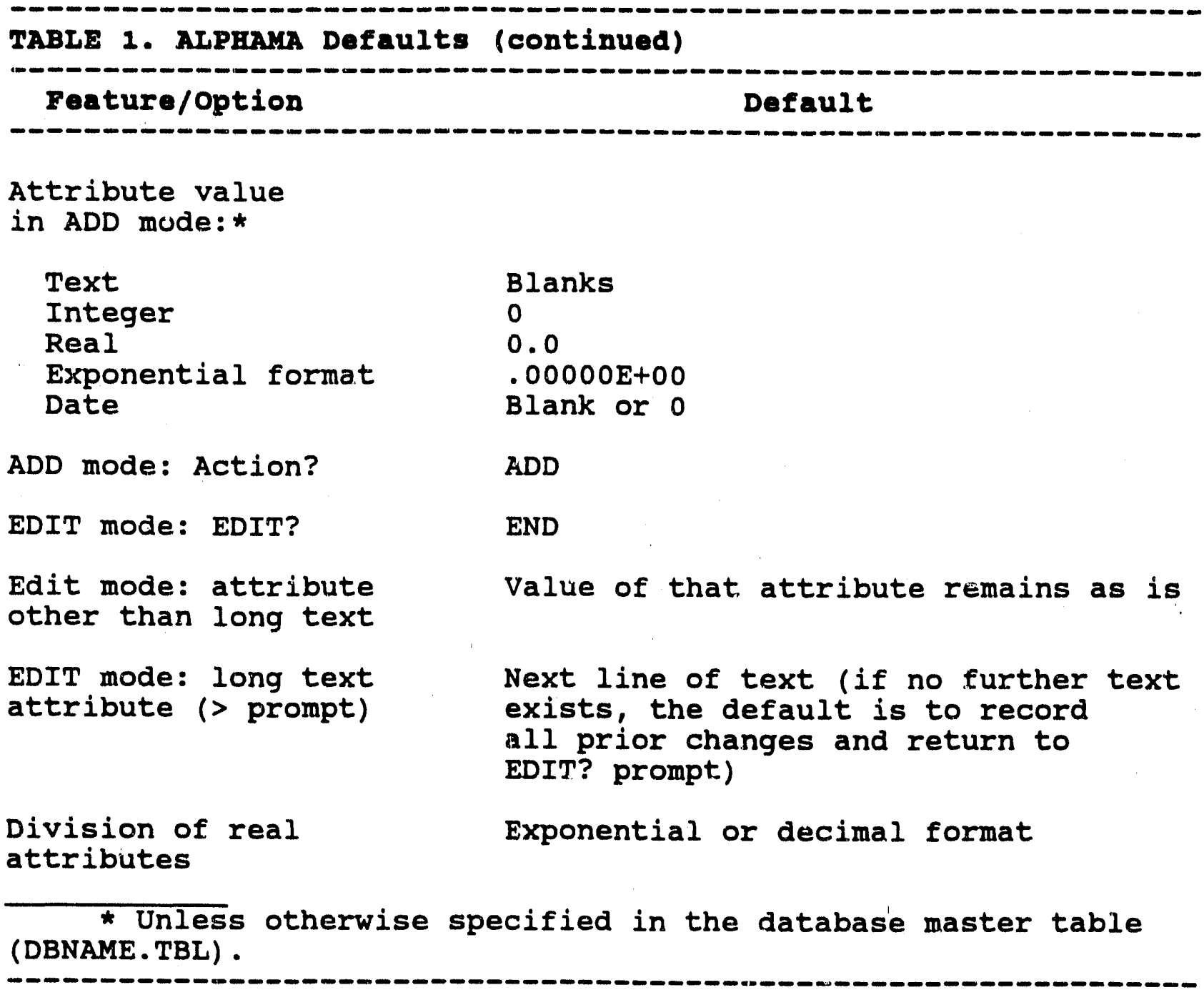

\subsection{A T T I B O T E 8}

\section{2 .1 T P 8}

ALPHAMA can maintain databases with both text and numeric attributes. Numeric attributes can be dates, integers, or real numbers.

\subsubsection{Text: short and Long}

ALPHAMA is able to maintain text attributes that contain up to 2400 characters (long text attributes). ALPHAMA edits text attributes that contain more than 15 characters differently than text attributes that contain fewer than 16 characters (short text attributes). 
Short text attributes, which have storage capacity of 15 characters or fewer, may include any character contained in a long text attribute except commas and spaces, unless the value containing commas or spaces is enclosed in double quotes (e.g., "SHORT TEXT"). All lowercase letters input to a short text attribute are converted to uppercase and stored as uppercase unless the input value is enclosed in double quotes.

Text attributes that contain more than 15 characters are considered long text attributes. ALPHAMA accepts and stores almost any keyboard character for a long text attribute. Uppercase and lowercase letters are preserved as entered. The only characters not

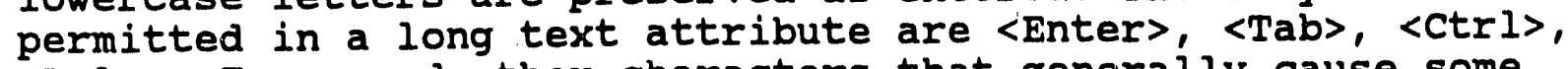
$<D e l>$, <EsC>, and other characters that generally cause some function to be executed. Commas, arithmetic operators (,,$- \ldots$, 1), double and single quotation marks (" or '), spaces, and other special characters $(!, e, z, \cdot,:, ;$, etc.) are acceptable. A discussion of ALPHAMA's editing capabilities for long text attributes is found in sect. $3 \cdot 7.2$.

\subsubsection{Dates}

ALPHAMA accepts the value of a date attribute in many different forms. Acceptable date formats are

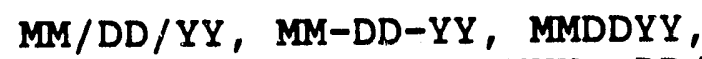
$M M / D D / Y Y Y Y, M M-D D-Y Y Y Y, D D / M O N / Y Y$, MON-DD-YY

where

$$
\begin{aligned}
& \text { MM = the numeric designation of the month } \\
& \text { MON = the first three letters of the month } \\
& D D=\text { the day of the month } \\
& \text { YY = the last two digits of the year } \\
& \text { YYYY = all four digits of the year }
\end{aligned}
$$

If only the montin and year (MM/YY or MM/YYYY) are entered, then ALPHAMA stores the date as the last day of the month and year entered; for example, $3 / 84$ results in the date March 31,1984 . Entry of dates in the form $\mathrm{DD} / \mathrm{MM} / \mathrm{YY}$ assumes the form $\mathrm{MM} / \mathrm{DD} / \mathrm{YY}$ unless the date is valid only in the form $\mathrm{DD} / \mathrm{MM} / \mathrm{YY}$. Users should avoid entering dates in the format $\mathrm{DD} / \mathrm{MM} / \mathrm{YY}$, because a date entered as $01 / 03 / 1984$ is stored as January 3, 1984, not as March 1, 1984 .

Entry of dates that cannot exist (e.g., 13/21/84 or 2/30/84) results is an error message, and ALPHAMA repeats a request for input. 


\subsubsection{Integers}

Any attribute defined as an integer accepts input of only numbers (0 through 9) and a sign. Neither decimal points, spaces, nor commas are valid characters within the number. Attempting to input an integer number and include a decimal point causes AIPHAMA to respond with an error message and repeat the request for the value of that attribute. In EDIT mode, including a comma or commas as the new value of an integer attribute $(e .9 ., 1,234,567)$ also results in an error message and a request for the new value of the attribute.

CAUTION: In ADD mode, a more dangerous situation exists in regard to commas or spaces, which are reserved as delimiters for use with stringed input. Instead of displaying a message, ALPHAMA processes the numbers -- storing each number(s) bounded by a commas as a separate attribute. For example, inputting 1,234,567 results in the value " 1 " being stored for the current attribute, the value "234" for the next attribute, and the value "567" for the next attribute. ALPHAMA then prompts for the value of the attribute following the attribute that stored the value of 567 .

\subsubsection{Real Numbers: Decimal and Exponential}

ALPHAMA divides real numbers into two categories: decimal and exponential. Decimal numbers must fit within the number of digits allowed without adjustment by an exponent. ALPHAMA accepts only numbers ( 0 through 9), a sign, and an optional decimal point as input to a decimal attribute. If a number exceeds the number of characters allowed or includes an " $E$ " ( $E$, exponential), ALPHAMA responds with an error message and repeats the request for the value of the attribute.

Exponential numbers must fit within the number of significant digits allowed for the attribute. The digits input must be shifted by an exponent. ALPHAMA accepts numbers (0 through 9), a sign for the number, a decimal point, the letter "E" (uppercase or lowercase, without opening and closing quotation marks), a sign for the exponent, and numbers for the exponent. Any other character results in an error message.

Commas are not allowed in either decimal or exponential numbers. With real numbers as with integers, commas function as delimiters. The caution in sect. 2.2.1.3 applies here as well. For example, if you want to give the value 85.6 to an attribute but actually type 85,6 , the value 85.0 is stored in the current attribute, and the value 6 is stored in the following actribute. 


\section{$2.2 .2 D \& i m i t \in \mathrm{s}$}

ALPHAMA invests certain characters with special characteristics. In ADD mode for all but long text attributes, a comma and a single space are delimiters that separate the value of an attribute from the value of the next attribute (see sect. 2.2.1.3). To include commas or spaces in a short text attribute, enclose the value in quotation marks (e.g., "value"; "value, value"). Exhibit 1 shows an example of a comma as delimiter (see Appendix B, sect. B.6, for the ADD list).

\section{EXHIBIT 1. Example of Comma Used as Delimiter in ADD Mode}

Display output

oser input

\begin{tabular}{ll} 
REAL1? & $85,6<$ Enter> \\
AD? & $4<$ Enter> \\
EF1? & <Enter> \\
EF2? & <Enter> \\
DN1? & <Enter> \\
Action? & EDIT<Enter> \\
EDIT? & REAL1<Enter> \\
REAL1 = 85.00 & \\
$?$ EDIT? & <Enter> \\
REAL2 $=6.00$ & REAL2<Enter> \\
$?$ & \\
\hline
\end{tabular}

\section{2 .3 D E f u 1 t}

See Table 1 for values that AIPHAMA assigns to attributes when the user presses <Enter> instead of inputting specific values. For example, in ADD mode, the default value for a text attribute is a blank; for an integer, zero (0). 
This page intentionally left blank. 


\section{C O M M A N D B A N D O P T I O N}

This chapter explains ALPHAMA commands as they might be used during a maintenance session. It is designed as a reference tool for typical users, as well as for users who desire to have a relatively high degree of familiarity with ALPHAMA or ALPHAMA-based programs. Proficiency with all of the features discussed here is not necessary for a general user.

\subsection{A C C E 8 S I N G T H E P R O G R A M}

To run ALPHAMA, a person must have a computer account (user identification number and authorization) on the Energy systems computer(s) where ALPHAMA resides (VAX computer system 61 and PDP10 systems 1 or 30 ); to run it on a VAX computer, a user mist also have Guide access. If you are such a user, you can access ALPHAMA from the VAX computer by typing at the dollar (\$) prompt:

\section{\$ ALPHAMA<Enter>}

To access from the PDP-10 system prompt (.), type:

\section{- DO ALPHAMA<Enter>}

Once in ALPHAMA (Exhibit 2), you can access any database for which you have update privileges (see sect. 3.4).

\section{EXHIBIT 2. Accessing ALPHANA}

\section{\$ ALPHAMA<Enter>}

ALPHAMA Maintenance Program - Version : V5C
Date: $8 / 27 / 91$
Tims: $14: 37$

For assistance contact: J. K. Lovin (6-2859)

: Suzanne Pratt (4-4683)

<Database>

\subsection{E X I T I N G}

\section{$3.2 .120 \mathrm{I}$}

QUIT is the command designed to take you out of ALPHAMA. It is a general, rather than a prompt-specific command, and thus can be executed at any prompt. It stores any changes made to the 
record, closes all database support files and the database, and takes the user to the system prompt (monitor level):

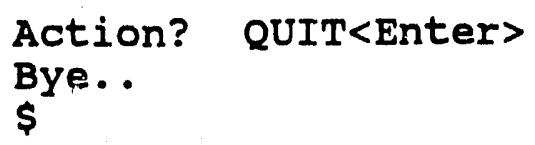

\subsubsection{END}

Another general feature is the command END. Typing END requests ALPHAMA to move the user back to the next highest (that is, the previous) level in the program. Thus when issued at the <Database> prompt, it takes the user out of the program. (Its effect when issued at specific prompts is discussed below with each command.)

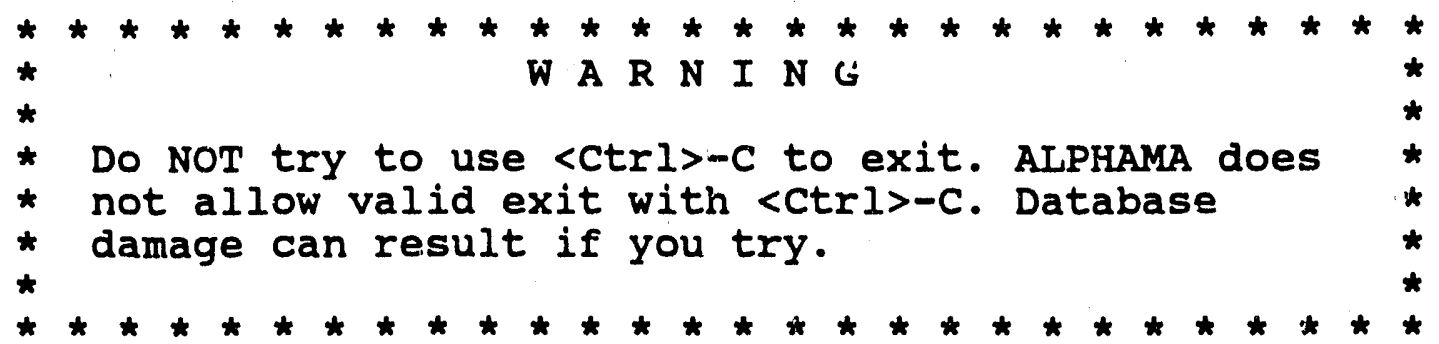

\subsection{O 8 I N G H E I P}

ALPHAMA provides users with online Help. The first level of assistance is the ALPHAMA prompts themselves, which query the user for input (e.g., ID1?). AIPHAMA also provides automatic Help (error) messages that respond to invalid input (e.g., see sect. 5.1, items 16 and 23). In addition to these unsolicited aids is online Help that the user can choose to access at any time.

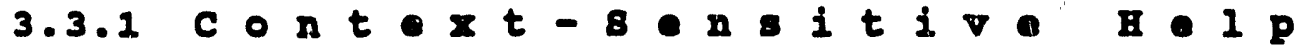

The most common type of assistance required by users is likely to be information on their current place in the program. Contextsensitive Help tells the user just what input is valid for the prompt on the screen. ALPHAMA provides two levels of such assistance, which is invoked when you press either one or two question marks (? or ??).

\subsubsection{Question Mark (?)}

As mentioned in sect. 3.3, the first level of Help is unsolicited: the ALPHAMA prompt itself. To access the second level of Help, however, enter a single question mark (?) . 
ALPHAMA's response is either a short message about the prompt or a brief (memory-jogging) list of valid responses. Exhibit 3 shows ALPHAMA response to the single question mark (?) at two different prompts.

EXHIBIT 3. Using second-Level Help

LONG prompting $(\mathrm{Y} / \mathrm{N})$ ? ?<Enter>

LONG prompting uses the full 25 character description to ask for items.

SHORT prompting uses only the 5 character abbreviation to ask for items.

\section{EDIT? ?<Enter>}

Editing commands are:

ABORT APPLY Att BATCH DELETE DISPLAY END QUIT

SAVE SKIP Att

\subsubsection{Double Question Marks (??)}

Double question marks (??) request third-level Help. ALPHAMA's response to this input consists of detailed information available from and referenced by the DBNAME.TBL file. Data displayed include templates and all available codes and names for coded data. When a user requests assistance for something other than an attribute, ALPHAMA displays the acceptable options at that point and a short explanation of each option.

For example, Exhibit 4 shows how ALPHAMA responds to two question marks (??) at the <Database> prompt by listing and explaining all the databases in the Information Center Index. As Exhibit 5 shows, typing two question marks (??) at the prompt for a unique identifier (sect. 3.5.1) elicits from ALPHAMA a full explanation of an appropriate response. In this instance, ALPHAMA describes the components (format) of a valid unique identifier value. 


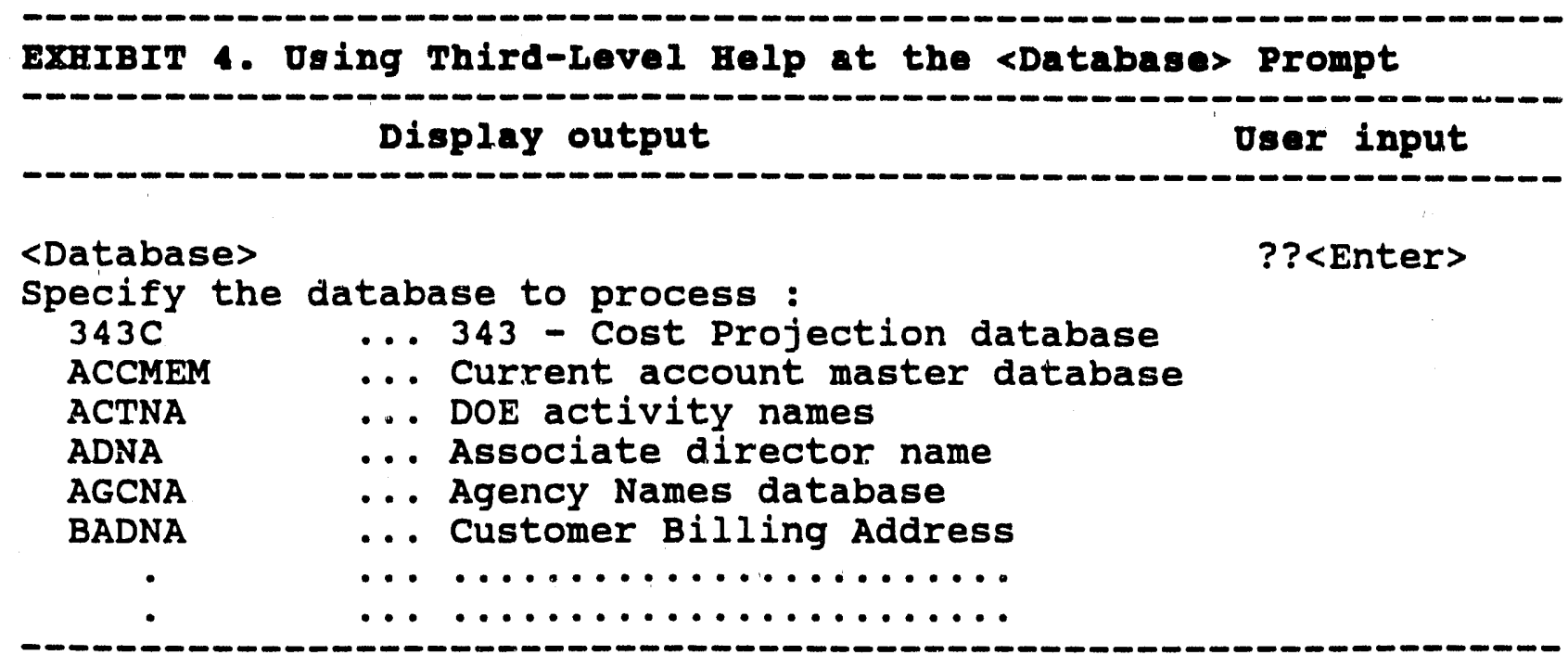

\section{EXHIBIT 5. Osing Third-Level Help at the onique Identifier Prompt}

Search for:

ID1?

ID2?
AB1234567<Enter> ??<Enter>

Data Type: Intg. Number of Characters: 2

Use template $2 \mathrm{~N}$

Where the numbers tell how many Numbers(N),

Letters(L), Spaces(S), Numbers or Letters(X), or Anything(A) make up the value.

ID2?

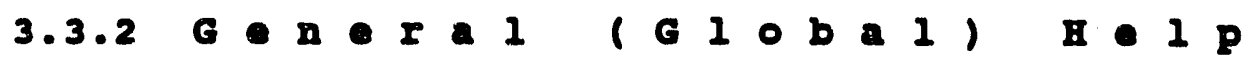

The fourth and highest level of assistance is provided by the HELP cormand. It provides detailed assistance on an item (an "argument") you specify; the request need not be tied in any way to the current prompt. A general command, HELP can be used virtualiy anywhere in the program. The request does not affect the program location or execution. It has two forms:

1. HELP Arg<Enter>

2. HELP Arg1 Arg2<Enter> 


\subsubsection{HELP Arg}

The single argument form does not restrict HELP to any type of information. Thus typing HELP SAVE<Enter>, for example, requests all information on SAVE, regardless of whether SAVE is a command, attribute, or database. The special features of HELP (using only Arg) option are listed in Table 2.

TABLE 2. Special Features of HELP Arg

Command

special feature

HELP Att

Lists detailed help on a user-specified attribute (Att); if the attribute references another database, ALPHAMA asks if it should display the values in the referenced database*

HELP ATTR Lists all the attributes in the database

HELP ATT?? Lists all the attributes and their long names

HELP HELP Prints the entire Help file

HELP SYS Prints online documentation for user-specified system (Sys) -- for example, ALPHAMA

HELP ?

Lists all arguments available in the Help file (attributes are not included)

ÆSee Sect. 4.3 .

\subsubsection{HELP Arg1 Arg2}

Users can narrow the field for ALPHAMA search by specifying both arguments. When Arg2 is provided, ALPHAMA limits Help to that specified by Arg1 -- which can be an attribute, operator, database, key word, command, or option:

Argument 1

ATtribute

DB

Command
Argument 2

Help is restricted to an attribute Help is restricted to a database Help is restricted to a command or an option

For example, typing HELP COMMAND SAVE<Enter> requests information on the command SAVE -- not on any other SAVE (e.g., not an attribute or database). 
The argument name can be either its full name (e.g., SAVE) or its ABBREVIATION (e.g., SA). All entries found in the Help file will be printed. When an abbreviation is entered, HELP provides all entries beginning with those characters.

\subsection{O P E N I N G A A D A I A B A B E :

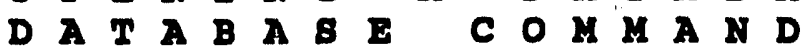

Once you access ALPHAMA, the next step is to identify for ALPHAMA which System 1022 or 1032 database you wish to open -- that is, the database whose records will constitute your selection set. The <Database> prompt on the screen (Exhibit 2) indicates that ALPHAMA automaticaliy executed its DATABASE (DB) command when you opened the program; it is ready for you to indicate which database to open.

On the VAX computer you can do so in two ways. (1) You can enter the full filename (directory path):

<Database> DEV:[DIRECTORY]FILENAME. EXT<Enter>

where

$$
\begin{aligned}
& \text { DEV } \\
& \text { DIRECTORY }=\text { Locice (usually the disk structure) } \\
& \text { FILENAME }=\text { Name of the database } \\
& \text { EXT }
\end{aligned}
$$

Or, (2) if the database is on the Information Center Index (INDEX.DMS file) or an INDEX.DMS file in your area, you can enter its Index short name, or "filecode" (pointing to its full path and filename):

<Database> FILECODE<Enter>

If you do not know a database filecode, type one or two question marks (? or ??) at the <Database> prompt; ALPHAMA will list all of the filecodes on the Index. As an example, to access the BUYER.DMS database, you could type either its full path (1) or filecode (2):

1. <Database> U1:[GUIDE_AREA.GENERAL]BUYER.DMS<Enter>

2. <Database> BUYER<Enter>

When you type a filecode and not the filename and full path, ALPHAMA assumes that the code is listed in an INDEX.DMS file in your own area (directory) and looks for it there. If ALPHAMA does not find an INDEX.DMS or a filecode that matches the input, it searches next in the Information center INDEX.DMS. If it finds the code, it reads the file specification described chere and opens the database. If the preceding fails, ALPHAMA checks your 
current directory for a filename and extension (e.g., MYDATA.DMS).

If you do not have access to maintain the database you specify, ALPHAMA will display an error message and repeat its request for a database specification. If you do have authorization and ALFHAMA locates and opens the database, it also opens any other databases associated with it (see Exhibit 6).

EXIIBIT 6. Specifying a Database

Display output and user input

Process

<Database> U1:[GUIDE_AREA.GENERAL]BUYER.DMS<Enter>

a

- . Opening database U1: [GUIDE_AREA.GENERAL]BUYER.DMS

Support file U5: [SP4.PTS]PTS.DMS was processed.

b

LONG prompting $(\mathrm{X} / \mathrm{N})$ ? $\mathrm{Y}<$ Enter $>$

c

Do you want to see default values displayed $(Y / N)$ ? $Y<$ Enter> d

5 records available

e

Search for:

$\mathbf{f}$

NOTE:

$a=$ User specifies a database.
$b=$ ALPHAMA responds and opens (processes) an associated file.

$c=$ User requests full atiribute names as prompts.

$d=$ User requests display of values as they apply to attributes.

$e=$ ALPHAMA displays the number of active records in BUYER . DMS .

$f=$ ALPHAMA moves into SEARCH mode so user can select a record.

ALPHAMA provides the option to change the database being updated without exiting the ALPHAMA program. When ALPHAMA prompts for a unique identifier (see item $f$ in Exhibit 6) to begin its search for a record, user response can be the Database (DB) command instead. ALPHAMA will then display the <Database> prompt. To open a new database (and thereby close the current one), enter the filecode or full filename specification of the database just as you did when you first accessed ALPHAMA and saw the <Database> prompt. 
3.5 S E I E C T I NG RECOR D 8 :

B E R C H MO D E

As soon as you open a database, ALPHAMA takes you to the SEARCH mode, where you can proceed to select each record of interest. ALPHAMA allows you to add or edit one record at a time and thus prompts you to identify the particular record you wish to add or edit by a collection of attributes that make it unique in the database (i.e., a unique identifier, or UID). This will be especially easy to do if the database has a master table listing one or more UIDs for the darabase (see sect. 4.2).

If you need information about records in the database before making your selection, ALPHAMA can provide it. At any SEARCH mode UID prompt except the first, you can use the LIST option to view UID values in selection set records or use the VALUE option to see the number of records in the set, as well as the unique values for the current UID. (If you wish to cancel a search while in this mode, you can enter ABORT; ALPHAMA will cancel any current search and return to the search for: prompt.)

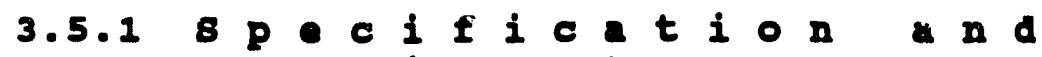 $R \cdot t r i \bullet \nabla=1$ ( 0 I D )}

At initial entry into the SEARCH mode, ALPHAMA checks to see if a master table exists for the database you opened. ALPHAMA searches it for the attributes required to identify a record uniquely. If ALPHAMA does not find UIDs in the database master table or if no master table exists, the user must input the UID attributes (see Exhibit 7); ALPHAMA accepts up to 10. (NOTE: To terminate selection and return to the <Database> prompt, use the ABORT command.)

\section{EXgIBIT 7. Belecting Records with Onique Identifiers}

Please input the ATTRIBUTE(s) that uniquely identify a record. For example:

UNIQUE IDENTIFIERS ? attr1, attr2,attr3, END (Identifies a record by 3 attribute names.)
ALPHAMA prompts for attributes.
ATTRIBUTE 1? ID1<Enter>
ATTRIBUTE 2? ID2<Enter>
User inputs UID
ATTRIBUTE 3? ID3<Enter>
attributes.
ATTRIBUTE 4? ID4<Enter>
ATTRIBUTE 5? <Enter> User ends list. 
Whether ALPHAMA finds UIDs in a master table or the user has to enter them, identifying the target record by attribute(s) is the first step in selecting a record. Next ALPHAMA prompts for unique attribute values (e.g., AB1234567, NEW1, 12, and 1):

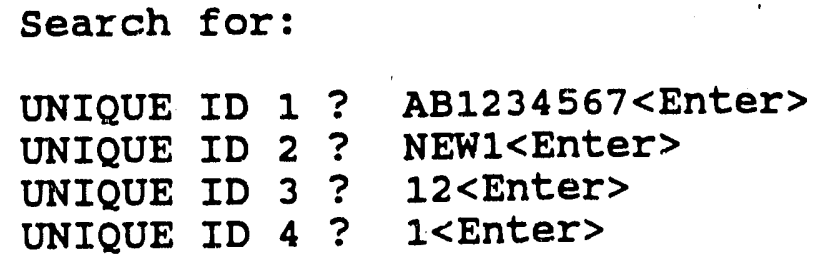

It then attempts to find the record whose UIDs match these values. (NOTE: For long text attributes, ALPHAMA searches on only the first 15 characters.) If ALPHAMA does not find the record, it asks the user whether or not to add a record (sect. 3.6). If ALPHAMA does find the record, it invokes the EDIT mode (Sect. 3.7).

occasionally ALPHAMA finds more than one record with the same value for each of the UIDs. This does not happen often; but when it does, AIDHAMA displays a message of the duplication. You can then view the records and take appropriate action. You can delete the unnacessary record(s) (Exhibit 8 ) or change one or more of the UIDs so that the duplication no longer exists. Adding UIDs to make the selection scheme unique will eliminate duplicate selections.

EXHIBIT 8. Deleting Duplicate Records

ALPHANA prompts and user input Process

ATTRIBUTE 1? ID1<Enter>

ATTRIBUTE 2? ID2<Enter>

Found 2 records with that ID
User inputs UID values.

ALPHAMA message indicates existence of duplicate IDs (2).

Would you like to see record \#1? No<Enter> ALPHAMA asks whether to display duplicate \#1; user says no.

ALPHAMA asks whether uses wishes to edit it; user says no.

Would you like to see record \#2? No<Enter>
ALPHAMA queries; user responds no. 
EXHIBIT 8. Deleting Duplicate Records (continued)

$\begin{array}{ll}\text { Would you like to edit it? } & \text { YES<Enter> } \\ \text { EDIT? DELETE<Enter> } & \begin{array}{l}\text { ALPHAMA queries; } \\ \text { user responds yes. }\end{array} \\ & \begin{array}{l}\text { At prompt, user } \\ \text { enters the DELETE } \\ \text { command. }\end{array}\end{array}$

To avoid creating duplicate records, maintain system 1022 or 1032 databases through ALPHAMA instead of working directly with System 1022 and 1032. Then ALWAYS provide ALPHAMA with the same UIDs when specifying a record to search for (whether you input them at every session or create a master table (DBNAME.TBL), a single source, to provide them].

\subsection{2 $\mathrm{E} \bullet \mathrm{I}$}

\section{5 .2 .1 IIBT}

Invoking the IIST option during SEARCH mode causes ALPHAMA to display UID values in selection set records. This option is valid for all SEARCH mode UID prompts except the first (at the first prompt, the selection set is still the ENTIRE database). For example, to request a list of UID values for the records having the value AB1234567 for attribute ID1, type LIST at the ID2 prompt:

Search for:

\begin{tabular}{ccc} 
ID1? & \multicolumn{3}{l}{ AB1234567<Enter> } \\
ID2? & LIST<Enter> \\
1 & ID1 & AB1234567 \\
& ID2 $:$ & 3 \\
2 & ID1 $:$ & $A B 1234567$ \\
& ID2 $:$ & 1
\end{tabular}

If you want attributes in addition to the UIDs, enter their names after the LIST command. ALPHAMA will display the UIDs plus the specified additional attributes (maximum of 10). For example, to request attributes REALI and INT1, enter:

ID2? LIST REAL1 INT1<Enter>

The program will display the following list: 


$\begin{array}{llll}1 & \text { ID1 } & : & \text { AB1234567 } \\ & \text { ID2 } & : & 3 \\ & \text { REAL1 } & : & 1234.560 \\ & \text { INT1 } & : & 12345 \\ & \text { ID1 } & : & \text { AB1234567 } \\ & \text { ID2 } & : & 1 \\ & \text { REAL1 } & : & 4567.890 \\ & \text { INT1 } & : & 45678\end{array}$

After displaying attributes for each record, ALPHAMA asks which record(s) you wish to edit. If you do not want to edit any, press <Enter>; ALPHAMA will return the cursor to the previous UID prompt. If you do wish to edit one or more records, enter the string of numbers designating which ones [the list number preceding the attribute name identifies the record(s)]. ALPHAMA will take you to EDIT mode with the first record selected. As you finish editing each record, ALPHAMA stores it and moves the cursor to the next specified record (still in EDIT mode).

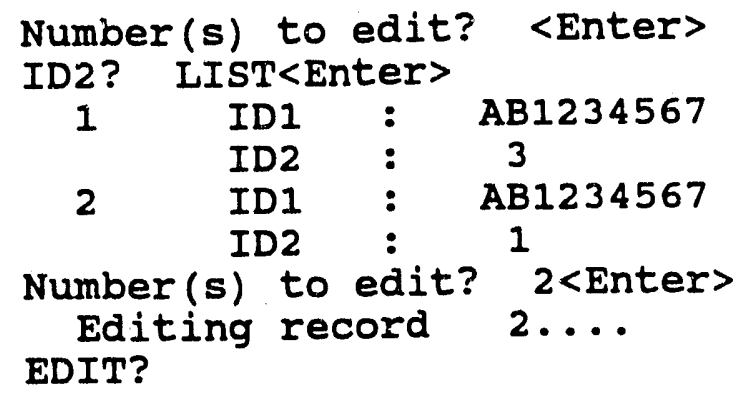

[NOTE: While in the EDIT mode you may use the DISPLAY option (Se=t. 3.7.3) to view all of the attributes in a chosen record.]

\section{5 .2 .2 VALUE}

VALUE is another Help option available during SEARCH mode at the prompts for UIDs. It displays the number of records in the selection set and the unique values for the current UID in the selected records. Like the LIST option, VALUE is invalid at the first UID prompt (when the selection set is still, the entire database); if you enter the option there, ALPHAMA will display an error message and then repeat the prompt for the first UID -- as shown in the example below:

ID1? VALUE<Enter>

VALUE is not a valid command for the first UID.

IDI? AB1234567<Enter>

ID2? VALUE<Enter>

ID2

2 records found.

$\mathrm{N} / \mathrm{A}$

TEST

ID2? 
The user has the option to enter the ADD mode when the input UIDs do not match the record values of an existing record. If the user chooses to add the record, ALPHAMA prompts for the value of each attribute in the ADD list. If no ADD list (DBNAME.ADD) exists, ALPHAMA assumes the ADD list consists of all attributes in the database in the order the attributes appear in the data description file (DBNAME.DMD).

The Add? prompt indicates that ALPHAMA has invoked the ADD mode. Table 3 lists the options available at the Add? prompt.

TABLE 3. Options at the ALPHANA Add? Prompt

Begins adding a record
Begins adding a record using the LAST RECORD added
Or edited as the source of the default value

When a user enters END, QUIT, ADD, or <Enter> at the Action? prompt following the input of data, ALPHAMA adds the record to the database. (See sect. 5.1 for an example Add session.)

\section{$3.6 .18 t I i n g e d I \mathrm{~g} u t$}

While in ADD (not EDIT) mode, ALPHAMA accepts stringed input: values of several attributes strung together in a single entry. only text attributes that contain more than 15 characters cannot be included in such an ADD string. After ALPHAMA processes UIDs, the user can enter the values of several attributes on a single line by using a comma or space to separate the value of one attribute from the value of the next. ALPHAMA assumes that the attributes follow the ADD list. After storing all values for the attributes included in the string, ALPHAMA prompts for the 
value of the next attribute in the ADD list. As an illustration, if the $i$ tem in the example ADD session (Sect. 5.1 and Appendix B, sect. B.6) is input as

INT1? $123000,1234.567,3456.7$ <Enter> ,

then

$$
\begin{aligned}
& \text { INT1 }=123000 \\
& \text { REAL1 }=1234.567 \\
& \text { REAL2 }=3456.7
\end{aligned}
$$

and the next prompt is for the attribute $A D$ :

$A D ? \quad$.

(NOTE: Only exrerienced ALPHAMA users should enter strings.)

\section{6 .2 I A 8 T}

IAST (IA) is an ADD option that tells ALPHAMA to use the values from the previous (last) record added or edited as the default values instead of those from the master table DBNAME.TBL. This option is useful if you are adding a series of records that are similar or identical except for their UIDs. Each time ALPHAMA prompts for whether you wish to add a record, enter IAST (Or IA) instead of YES (Table 3 ).

\subsubsection{K I P a d 8 KI P A $t$}

SKIP is a time-saving option available during ADD mode at any attribute prompt. It works in conjunction with the ADD list (DBNAME.ADD) and SKIP list (DBNAME.SKP) to display only specified prompts for a group of attributes and to skip over the others. Execution of the SKIP option causes ALPHAMA to pass over attributes in the ADD list until the next attribute in the SKIP list that matches an attribute in the ADD list appears. ALPHAMA then prompts for all remaining attributes in the ADD list (unless SKIP is executed again). If no SKIP list exists, the ADD list becomes the default for DBNAME.SKP. Default values are set for all attributes skipped (SKIP list, sect. 4.7; ADD list, sect. 4.6). For example, if the SKIP option had been entered in the ADD example (Sect. 5.1 and Appendix B, sects. B.6 and B.7), the results would have been:

$\begin{array}{ll}\text { TEXT1? } & \text { SKIP<Enter> } \\ \text { INT1? } & 12300<\text { Enter> } \\ \text { REALI? } & \text { SKIP<Enter> } \\ \text { AD? } & \text { SKIP<Enter> } \\ \text { AD? } & \end{array}$


Usually the next attribute prompted for in ADD mode after TEXT1 is TEXT2 (see Appendix B, sect. B.6: TSTMA.ADD). But SKIP entered at the prompt for TEXT1 tells ALPHAMA to skip to the next attribute listed in the SKIP list (see Appendix B, sect. B.7: TSTMA.SKP), INT1, and prompts for a value. When reentering the normal ADD mode, a response of SKIP at the prompt for REALI causes ALPHAMA to skip to the next attribute (AD) in the SKIP list. In this case, because the list has no more entries, a skip at the $A D$ prompt causes ALPHAMA to reprompt for the same attribute (AD).

SKIP followed by an attribute name causes ALPHAMA to skip over attributes in the ADD list until it reaches the specified attribute. (NOTE: You can also skip "backwards" to change a value of an attribute already entered: Just enter a previous attribute in the ADD list.)

\subsubsection{A u to o p a to}

Another feature available in the ADD mode (and in the EDIT mode, Sect. 3.7.6) is auto update, a good example of the benefit of maintaining databases with support files. When you enter a value for a "referenced" attribute [i.e., an attribute designated in the master table (DBNAME.TBL, sect. 4.2) as mapped or referenced to a support database], ALPHAMA looks at the corresponding support database listed in the database reference file (DBNAME.REF) (Sect. 4.3) for a record in which the referenced attribute has the same value. If ALPHAMA finds such a record, it retrieves from the support database the values of all attributes shared between the two databases and places them in the main database. Such automatic updating reduces input keystrokes and ensures consistent values across databases with shared attributes.

As an example, suppose a user has opened ADATA.DMS to add a record, and ADATA.DMS has the support file BDATA.DMS. The two databases contain the attributes listed below, and no two records have the same value for attribute $A C$, which is referenced to the database BDATA:

\begin{tabular}{ll} 
ADATA & BDATA \\
\hline ID1 & --- \\
ID2 & ATT1 \\
AC & AC \\
BR & ATT3 \\
FT & FT \\
ATTF & ATT5 \\
ATTG & BR \\
& ATT7 \\
& ATT8 \\
& ATT9
\end{tabular}


When the user gives ADATA attribute $A C$ a value that matches its value in BDATA, ALPHAMA automatically inputs the BR and FT attribute (common to both databases) values found in BDATA. An attribute will NOT be set, however, if

1. the attribute is a UID;

2. no record in the support database matches;

3. more than one record in the siupport database match;

4. the master table flag for an attribute indicates not to set, that is, the no-retrieve-on-map flag is 2 or 1 and the existing value is not blank or zero (Sect. 4.2); or

5. the value of the attribute in the support database is blank or zero.

In these cases, ALPHAMA prompts for a value.

\section{6 .5 s $t$ a $r$ (*) M u 1 t 1 i $i r$}

Star multiplier ( $n *$ value) is another ADD mode feature that saves keystrokes. With it, users can issue a single command to add a value to several attributes. ALPHAMA requires, however, that the value be the same for all attributes, and the attributes must be consecutive.

(Att prompt)? $n *$ VALUE<Enter $>$

where

$$
\begin{array}{ll}
\text { (Att prompt)? } & =\text { ADD mode attribute prompt } \\
\mathbf{n} & =\text { Number of consecutive attrioutes } \\
\text { VALUE } & =\text { Attribute value }
\end{array}
$$

The next " $n$ " number of attributes in the ADD list receive the entered value.

\section{$3.6 .6 \mathrm{~A} \subset t i \circ \mathrm{n}$ ? P r $0 \mathrm{mpt}$}

The Action? prompt appears after the user irputs the values for all attributes in the ADD list for a record. The user has the opportunity to see (using DISPIAY) the record as it currently exists, to add the record as it currently exists (default), to EDIT the record, or to ABORT the addition of the record to the database. No record is added to the database until the user issues the command $A D D$, <Enter>, END, or QUIT at the Action? prompt. 


\subsubsection{DIBPIAY}

DISPLAY allows viewing the current values of all attributes in the record being added. After the user views the record and presses [Enter], ALPHAMA redisplays the Action? prompt. (DISPIAY is also available in EDIT mode at the EDIT? prompt; see sect. 5.2 , item 16, for sample output.)

\section{$3.6 .6 .2 \quad A D D$}

ADD is an option that adds the current record to the database being maintained; it is the default response at the Action? prompt. Thus users can either enter ADD at the Action? prompt (see item 34 of the example ADD session in sect. 5.1) or simply press <Enter>.

\subsubsection{EDIT}

EDIT is an appropriate response when the user determines that some values were input incorrectly. It allows correcting the value of any attribute in the record being added. After the user corrects the attributes containing input errors, the Action? prompt reappears. Item 30 of the example ADD session (Sect. 5.1) provides an example of this capability. All EDIT mode options (see sect. 5.2) are then available to the user.

\subsubsection{ABORT}

ABORT is the only option available at the Action? prompt that telis ALPHAMA to discard the record just entered. EDIT takes the user to the EDIT mode, and all other options at the Action? prompt lead to addition of the record to the database. Entering ABORT causes ALPHAMA not to add the record and to take the user back to the UID prompt:

\section{Action? ABORT<Enter> \\ Search for:}

ID1?

\subsubsection{END and <Enter>}

The END command indicates completion of the specific add task. Executing END either by entering the command or pressing <Enter> adds the record to the database and returns the user to the Search for: prompt. 


\section{6 .6 .6 QUIT}

QUIT is a general command option to exit ALPHAMA (sect. 3.2.1). It closes the database and all its support files and takes the user to the system prompt (monitor). However, when issued at the Action? prompt, QUIT first adds the record to the database before proceeding to quit the session. If you decide not to add a record, you must use ABORT and then execute QUIT.

\subsection{E D I T I N G R E C O R D B: \\ E D I T MO D E}

ALPHAMA provides two ways to access the EDIT mode: (1) ALPHAMA automatically invokes EDIT mode if the user enters a record UID that matches an existing record, and (2) the user can enter the EDIT command at the Action? prompt. When the EDIT mode is active, ALPHAMA displays the EDIT? prompt. There the user can enter the attribute to edit, and ALPHAMA will display its current value. Next ALPHAMA prompts the user for the new value. If the user does not type in a new value but presses <Enter>, ALPHAMA retains the current value of the attribute. Section 5.2 contains an example EDIT session.

\subsubsection{8 h $5 t$ I $\quad x t$ A $t r i b u t e s$}

ALPHAMA editing of short text attributes (15 characters or fewer) is different from that of long text attributes ( 16 characters or greater). When users edit short text attributes, ALPHAMA converts all lowercase input to uppercase.

ALPHAMA allows all characters -- although values containing the comma and space (blank) must be enclosed in double quotes. To edit a short text attribute, request the attribute and then input the new value. To set the value to blanks, enter a pound sign (\#).

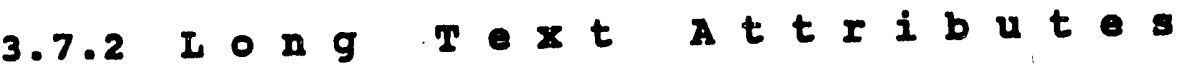

When adding or editing an attribute containing more than 15 characters, uppercase and lowercase characters are stored exactly as entered. In the ADD mode, all characters typed into a long text attribute are stored. Pressing <Enter> signals ALPHAMA that input for the attribute is finished. When the user reaches the end of a line, the cursor begins a new line. If the end of a line occurs in the middle of a word, the user should keep typing. (NOTE: ALPHAMA allows input from a terminal set to a width of 80 or 132 characters.)

ALPHAMA stores the text just as it is typed, not as it appears on the screen. ALPHAMA automatically divides the words at 
appropriate places; that is, words break properly when displayed, even if they appear divided on input. If input contains more than the maximum number of characters allowed for the attribute, all characters inputted after the maximum are not stored. Long text attributes may not be added using stringed input. No provision for paragraph breaks in a single, long, text attribute exists. If paragraph breaks are required, separate text attributes must exist for each paragraph.

When users edit a long text attribute, ALPHAMA displays the first line of text in the attribute and prompts with a "greater than" (>) character. Several options are available at this prompt. In the discussion of each option below, the current line of text is the line most recently displayed.

\subsubsection{8HOW}

SHOW displays the long text attribute, with a line number preceding each line of the attribute. Then the long text attribute, the line that was input, is returned for editing.

\section{$3.7 .2 .2<\#>$ (Delete value)}

A <\#> (pound sign) deletes the attribute value and replaces it with blanks. For example, the \# command issued below deletes the preceding displayed value for the entire attribute:

to the database. The database and all nicessary support files are

$>$ \#

No text to edit. Would you like to add some?<Enter>

Pressing <Enter> at the subsequent prompt confirms that the you wish to replace the value with blanks. On the other hand, if you want to enter a new value to replace the deleted one, you can do so at the prompt. Instead of pressing <Enter>, enter "Y" at the prompt, and type in the new value.

\subsubsection{3 <Enter> (Display Next Iine)}

An <Enter> by itself causes the program to display the next line of text. If no further text exists, any changes made to the attribute are stored, and the program returns to the EDIT? prompt.

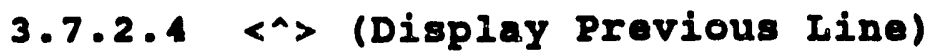

Inputting a caret ( $\left.{ }^{\wedge}\right)$ (<Shift-6> on most keyboards) causes the program to move to and display the previous line of text. 


\subsubsection{5 <D> (Delete Line)}

Inputting <D> causes the current line of text to bia heleted and the next line of text to be displayed. If deleting the current line results in deleting all text for the attribute, ALPHAMA asks if the user would like to add text.

\section{$3.7 .2 .6<I>$ (Insert)}

Inputting <I> invokes INSERT mode. Inserted text is placed in front of the current line of text. The current line of text and any text that follows are pushed down in the attribute. An <Enter> tells ALPHAMA that the user has finished inserting text. If inserted text causes the text in the attribute to exceed the space allowed, the excess amount is not stored. Item 25 of the example EDIT session (Sect. 5.2) demonstrates the use of INSERT.

\subsubsection{7 <A> (Append)}

Inputting $\angle A>$ invokes APPEND mode. The text appended in this manner attaches to the end of the text attribute being edited. If appended text causes the total number of characters in the attribute to exceed the maximum number of characters allotted, the excess amount is not stored. An <Enter> tells ALPHAMA that append activities are finished. Item 28 of the sample EDIT session (sect. 5.2) demonstrates the use of APPEND.

\subsubsection{8 <R> (Replace string)}

REPLACE $(<R>)$ permits altering strings of text in the current line of text. This option is particularly useful to correct misspelled words, add words or short phrases, and delete words or short phrases (sect. 5.1, item 26, is an example of REPLACE used to delete a phrase). The form of the command is

$$
\text { <R> (delimit)CURRENT_STRING (delimit)NEW_STRING (delimit) <Enter> }
$$

where

$$
\begin{aligned}
& \text { <R } \quad \text { = Replace option } \\
& \text { DELIMIT = Delimiter character signaling beginning } \\
& \text { and end of text to be replaced and to } \\
& \text { be inserted } \\
& \text { CURRENT_STRING = Text to be replaced } \\
& \text { NEW_STRING = Text to be inserted }
\end{aligned}
$$

The first character input after the $\langle R\rangle$ is used by ALPHAMA as a delimiting character. Any character may be the delimiter -- EXCEPT a character in either the current string or the new string: The slash (/) and asterisk (*) do not normally occur in text attributes and are good choices for delimiters. Execution of 
the REPLACE command causes the first occurrence of the "current string" in the current line to be replaced by the "new string." If the "current string" does not exist on the current line, no change is made.

For example, if a slash (/) is chosen as a delimiter and the misspelled word "computre" should be replaced with the correct spelling "computer," then the form of the command is

$$
<\text { R }>\text { / COMPUTRE/COMPUTER/<Enter> }
$$

REPLACE searches only the current line for the current string. If the current string exists more than once on the current line, the REPLACE command replaces the first occurrence only. REPLACE command must be repeated for each occurrence of the string on the current line. Items 21 and 22 of the example EDIT session (sect. 5.2) provide an example of text replacement.

\subsubsection{END}

Inputting END causes the program to store any changes made to the attribute and return to the EDIT? prompt. If the line being edited is the last or only line of text, an <Enter> invokes END automatically.

\subsubsection{I 8 P I A Y}

DISPLAY is a helpful EDIT mode option. Issued at the EDIT? prompt, DISPLAY lists the contents of the current record.

\section{$3.7 .4 \mathrm{At}$}

To indicate which attribute you wish to edit, type its name at the EDIT? prompt. ALPHAMA will make it current so you can proceec.

\section{7 .58 \& I P A $t$}

Entering SKIP plus the name of an attribute (Att) tells ALPHAMA to skip to a specified attribute and permit adding a selected group of items to an existing record. (See Sect. 3.6.3.) The command invokes ADD mode. At the end of the ADD list, ALPHAMA returns to the EDIT? prompt. 
3.7.6 A t $t$ o p a t e

The automatic updating possible in the ADD mode is also valid in the EDIT mode when you are editing a referenced attribute. See sect. 3.6.4 for how ALPHAMA can update a main database record with the up-to-date values of attributes shared in referenced support databases.

\subsubsection{A $E$}

SAVE retains the changes in a record for later use. The new values of changed attributes made prior to the execution of the SAVE command are stored in a temporary ("save") file. Attributes changed after the SAVE command has been executed will not be saved. Use SAVE when use values of a certain group of attributes must be changed to the same value(s) in more than one record (see APPLY, sect. 3.7.8). Only one save file exists, but it may be used as often as necessary.

\subsubsection{A P I I Y}

The execution of APPLY changes the values of all attributes in the record selected to the value of those same attributes in another record whose values were saved. Attributes in the current record that do not have a change in the save file are not affected. Exhibit 9 provides an example of user input to APPLY saved values to attributes in the selected record. The attributes TEXT1, TEXT2, and $A D$-- which were changed before the SAVE was entered -- now have the same value in both records. INT1 was changed after the SAVE and thus was not changed by the APPLY. (Any attribute may be edited after SAVE Cr APPIY is executed.)

\section{EXHIBIT 9. Using SAVE and APPLY}

Prompt

Osnr input

Search for:

ID1?

ID2?

ID3?

ID4?

EDIT?

TEXT1 = NEW VALUE

EDIT?
AB1234567<Enter>
NEW1<Enter>
$12<$ Enter>
$1<$ Enter>
TEXT1<Enter>

"SHORT ATT"<Enter>

TEXT2<Enter> 
EXHIBIT 9. Osing BAVE and APPLY (continued)

Prompt

oser input

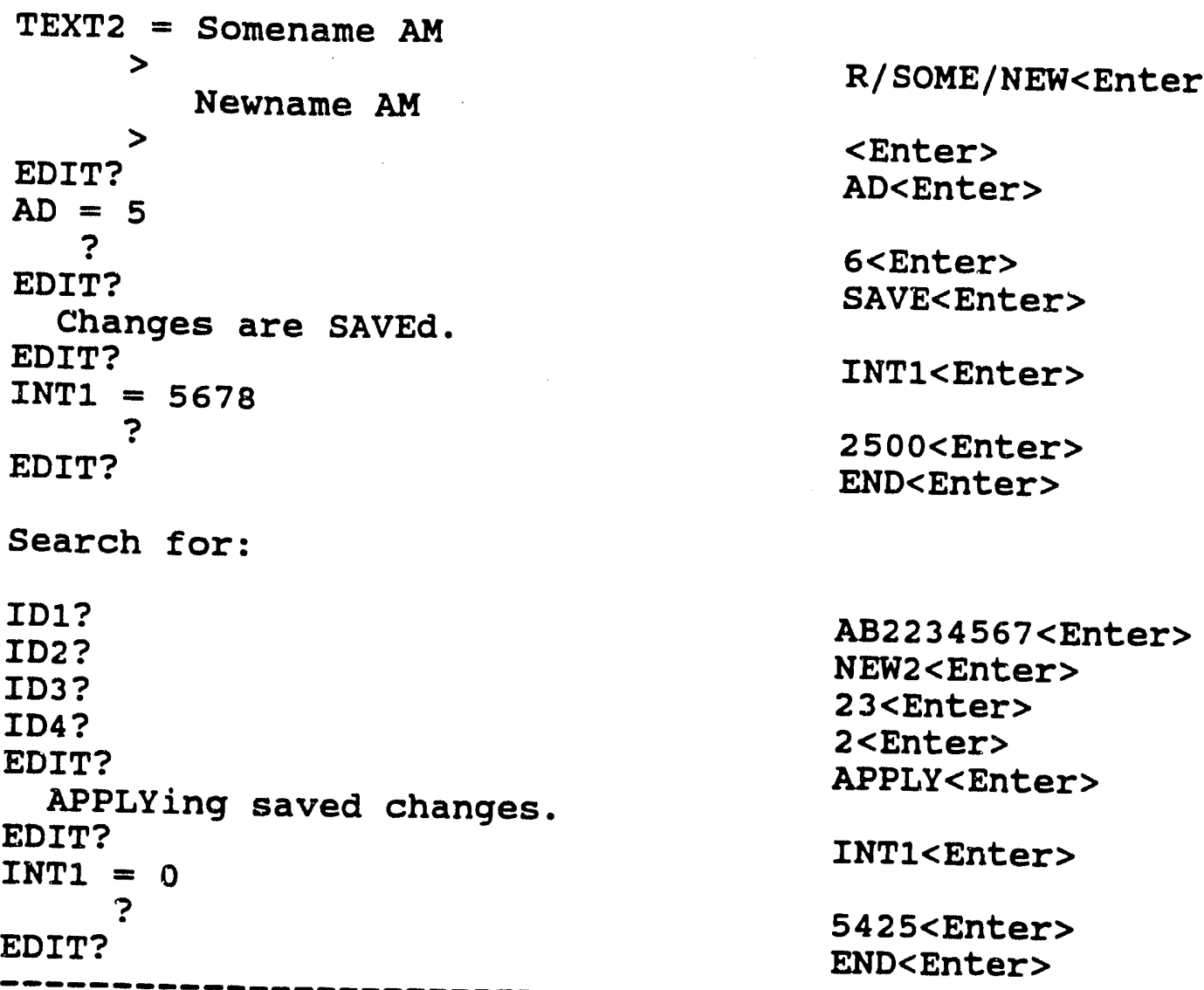

\subsubsection{E L E T E}

DELETE removes the currently selected record from the database. When a user issues this command, ALPHAMA requests verification that the record should be deleted; if the user confirms the deletion, ALPHAMA proceeds to delete it (Exhibit 10). section 5.3 provides an example DELETE session.

Deleted records may continue to exist in other systems and are therefore retrievable -- but not with ALPHAMA. If they have not been permanently disposed of by a dump and reload of the database, they can be located and accessed using the system 1022 and 1032 DFIND command. 
EXHIBIT 10. Deleting a Record

$\begin{array}{ll}\text { Search for: } & \\ \text { ATTRIBUTE 1? ID1<Enter> } & \text { User inputs UID } \\ \text { ATTRIBUTE 2? ID2<Enter> } & \text { values. } \\ \text { ATTRIBUTE 3? ID3<Enter> } & \text { User ends search; } \\ \text { ATTRIBUTE 4? END<Enter> } & \text { ALPHAMA goes to EDIT. } \\ & \\ \text { EDIT? DELETE<Enter> } & \text { User enters DELETE. } \\ \text { Are you SURE You want to DELETE } & \text { At confirm prompt, } \\ \text { this record? Y<Enter> } & \text { user says yes. } \\ \text { Record is DELETED from database. } & \text { ALPHAMA deletes the } \\ \text { Search for: } & \text { records and moves } \\ \text { to SEARCH mode. }\end{array}$

\section{7 .10 M VE ( P D P - 10$)$}

MOVE is a command unique to ALPHAMA on the PDP-10 computer (i.e., it is not available on the KSV VAX computer). It deletes the currently selected record from the active database and moves it to DBNAME.MOV (Sect. 4.8), which has the same structure as DBNAME.DMS. Moved records are still available in the main database through the System 1022 DFIND command until the database has been dumped and reloaded. Exhibit 11 describes an example of user input to execute the MOVE command.

EXIIBIT 11. Osing KOVE To Delete a Record on the PDP-10 Computer -..-Display output Oser input

Search for:

ID1?

ID2?

ID3?

ID4?

EDIT?

RECORD successfully MOVEd to new database.

AB1234567<Enter>

NEW1<Enter>

$12<$ Enter>

$1<$ Enter>

Search for:

MOVE<Enter>

ID1?

END<Enter> 
3.7.11 A B O R 1

ABORT cancels all changes made to the record during the edit session. The record remains as it was before the edit request. ALPHAMA exits the EDIT mode.

\section{7 .12 E N D}

Unlike ABORT, the END option stores any changes to the record in the database. Like ABORT, it takes the user out of the EDIT mode and returns to the search for: prompt.

\section{$3.7 .13 \quad 2 \quad 0 \quad I T$}

QUIT in the EDIT mode functions as it does in the ADD mode. First it stores any changes made to the record. Then it closes all database support files and the database and exits ALPHAMA. 


\section{B U P P O R T A N D A UXIIIARY FIIE}

This chapter describes the support and auxiliary files that make database maintenance more efficient. For example, you can specify the ALPHAMA prompt sequence of attributes by ordering them in an ADD file (DBNAME.ADD). You can establish a derivative list of the sequenced attributes in a "skip" file (DBNAME.SKP) so that during maintenance you can move directly to them. You can even set up index files that achieve automatic updating of matching attributes (sect. 3.6.4). For samples of these files, see Appendix $B$.

When you build a database for use with ALPHAMA-hased (and ALPHA-based) programs, you can also create these files. You can create a data description file (DBNAME.DMD), master table (DBNAME.TBL), and other support files (e.g., DBNAME.TEM, DBNAME.REF, DBNAME.SKP, DBNAME.ADD). Creating these files can be especially efficient if you use an Information Center program called DBBUILDER, which can help you build the database, data description file, and most of the remaining files. For more information on building databases and support files, see the ALPHA MIS Database Manual (Ref. 1).

\subsection{A T D E C R I P T I O N (DBNAME.DMD)}

The data description file, DBNAME.DMD, describes the data contained in the database. [Note: Data description files are more fully explained in the system 1022 Database Management System User's Reference Manual or System 1032 User's Guide (Refs. 5 and 6).] Every System 1022 and 1032 database has or had one. Initially System 1022 or 1032 uses the DMD file to create the database. Usually after the data input files have been loaded, the external DBNAME.DMD file is no longer used. Where applicable, the structure section of the data description file contains the features described in Table 4.

The values of certain attributes, including default values, may be required to be within a prespecified range of values. Range limits may be specified in either or both the data description (DBNAIE.DMD) or table (DBNAME.TBL) file. When the user attempts to input a value for an attribute outside the range specified, ALPHAMA responds with an error message containing the range limit violated and repeats the request for the value of the attribute. User response should be within range specifications in the master table file (DBNAME.TBL). If the master table, DBNAME.TBL, is not present, ALPHAMA uses the database internal structure to supply as much information as possible about the database. 


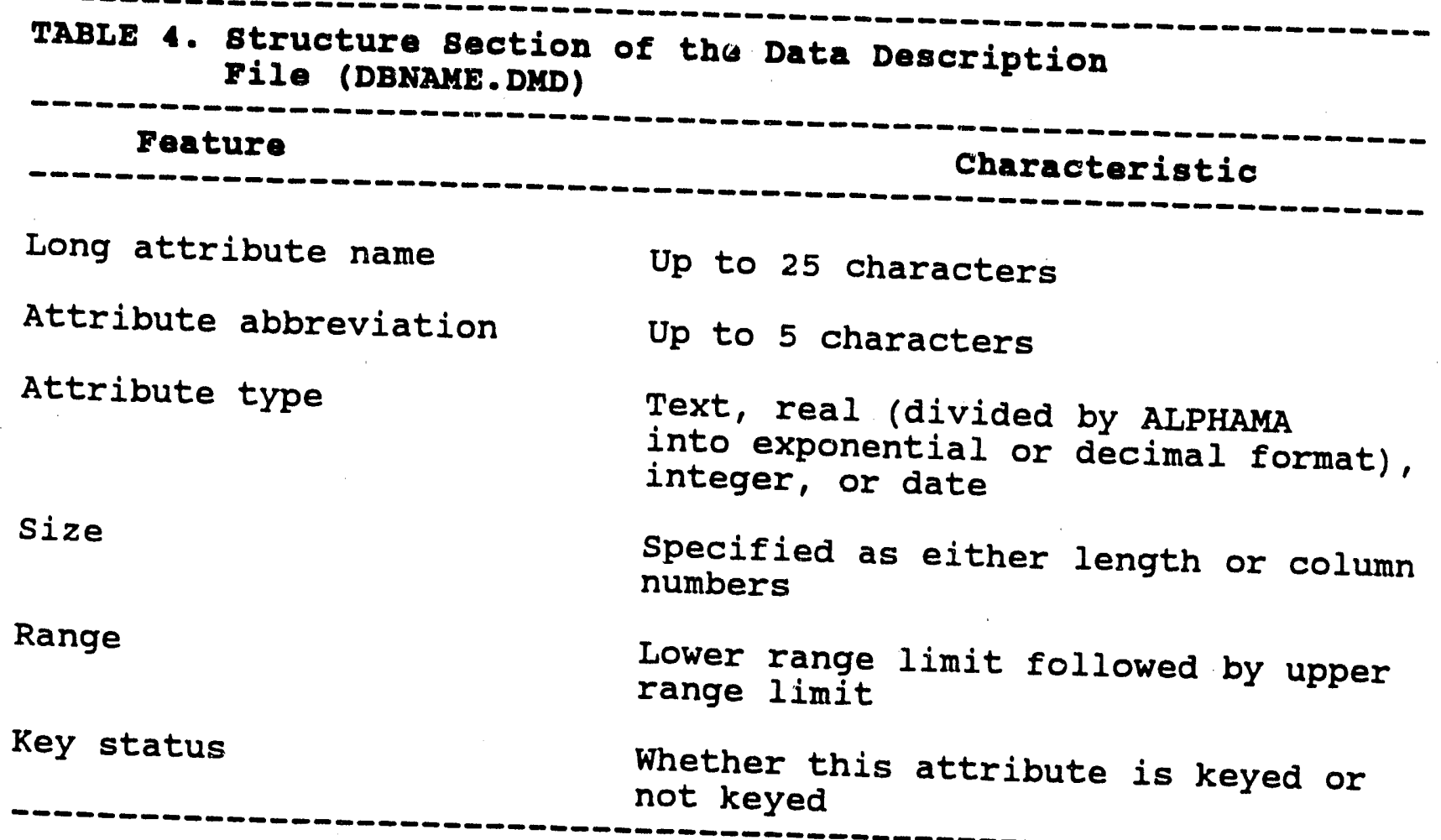

\subsection{A T E R T A B I E (DBNAME.TBL)}

The master table file, DBNAME.TBL, provides the capability to customize many maintenance functions without requiring applicationfiles, such as the ADD list table links ALPHAMA with other support the most important file in and reference and template files. It is (Table 5) are listed below.

TABLE 5. Contents of the Master Table (DBNAME.TBL)

(VAX) Characters

Column allowed in table*

Function

$\begin{array}{cl}1-5 & \text { A5 } \\ 7-9 & \text { I3 } \\ 11-14 & \text { I4 } \\ 16-19 & \text { I4 } \\ 21-30 & 2 A 5 \\ 32 & \text { I1 } \\ 34-65 & 3 A 5 \\ 67-98 & 3 A 5 \\ 100-131 & 3 A 5 \\ 133-135 & 03 \\ 142-143 & \text { I2 }\end{array}$

Attribute abbreviation

Attribute number

Number of characters

Number of words

Output format

Template flag

High-range limit

Low-range limit

Default value

Protection code

MAP (reference) code 
TABLE 5. Contents of the Master Table (DBNAME.TBL) (continued)

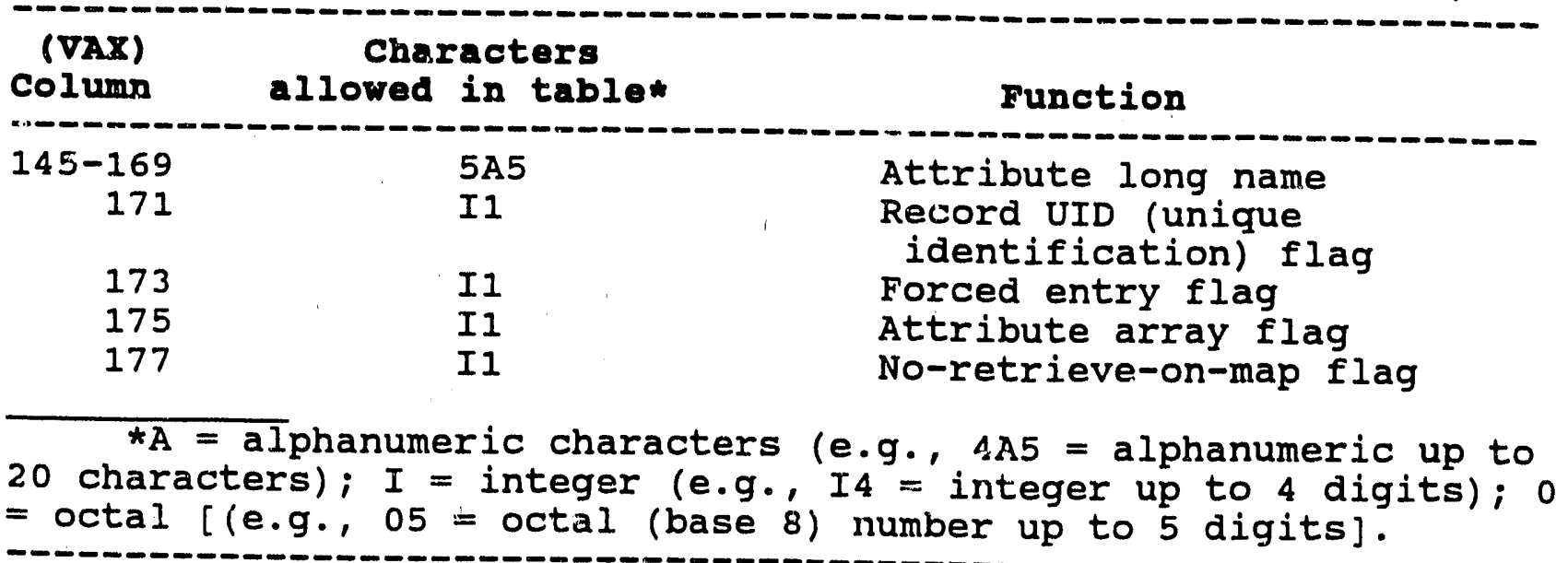

1. ATTRIBUTE ABBREVIATION: The attribute abbreviation used by ALPHA and ALPHAMA-based programs to identify the attribute -- the attribute abbreviation is usually the same as found in the data description file. The master table provides the mechanism to change the abbreviation used by ALPHA and ALPHAMA users without changing the database. This feature is particularly useful if the abbreviation must be changed immediately. The attribute abbreviation may be five characters or fewer. No two abbreviations in the same database may be exactly alike.

2. ATTRIBUTE NUMBER: The sequence number of the attribute as found in the data description file. Attributes must be sequenced in the same order as they are in the DBNAME. DMD file.

3. NUMBER OF CHARACTERS: The maximum number of characters that may be stored in the specified attribute -- For integer and real attributes the sign and decimal point are included in the number of characters. The number must be less than or equal to the size of the attribute as defined in the data description file.

4. NUMBER OF WORDS: The number of computer words (five bytes per word) required to store the specified attribute.

5. OUTPUT FORMAT: The format description used to output the value of the attribute.

6. TEMPLATE FLAG: Flag used to determine if attribute is forced to conform to one of a set of templates - - Templates are found in a template file (DBNAME.TEM):

$0 \ldots .$. No templates exist for this attribute

$1 \ldots .$. Templates exist in DBNAME.TEM for the attribute 
7. HIGH-RANGE LIMIT: The highest or greatest value that the attribute is allowed to contain (may be specified either in the data description file or, as here, in the master table file) -When a user attempts to input a value larger than the value specified here, ALPHAMA responds with an error message containing the range limit violated and repeats the request for the value of

8. LOW-RANGE LIMIT: The lowest or smallest value that the attribute is allowed to contain (see item 7 ).

9. DEFAULT VALUE: The value for the attribute (in ADD mode) if the user skips the attribute or presses <Enter> -- Specified defaults greater than 15 characters are not permitted. Unless otherwise specified the defaults are as follows:

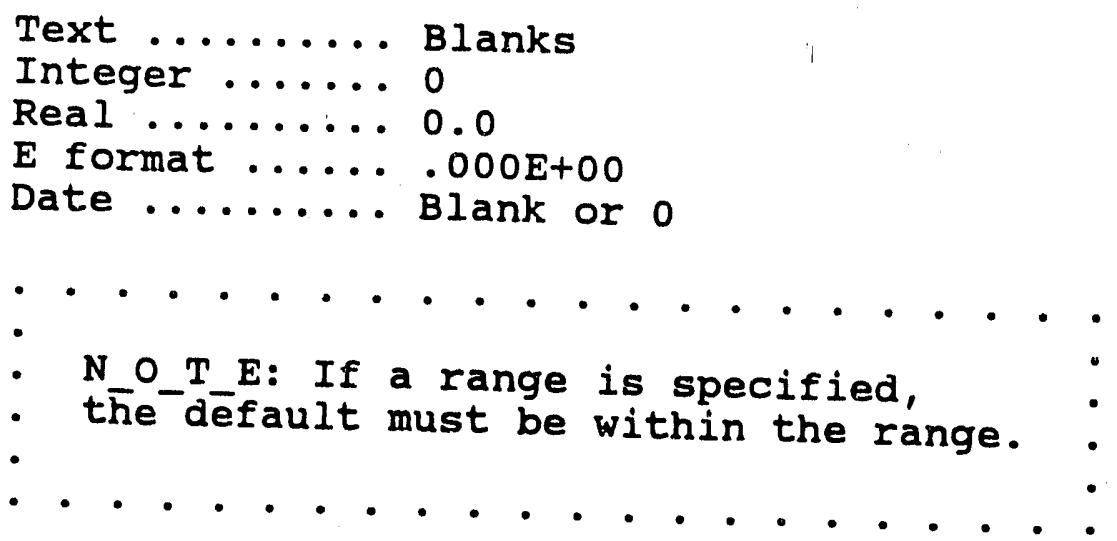

10. PROTECTION CODE: An octal (base 8) code used to disallow certain user access functions to the attribute -- The usual setting setting disallows displaying add exclude any functions. Any other

11. MAP (reference) code: Code used to indicate if the attribute is used to link the database with a referenced database - Zero (0) indicates that this attribute is not tied to a the refer database. A number other than 0 is the record number in specification file (DBNAME.REF), which gives the full file indicates that the referenced database. For example, a code of 3 third entry in the attribute maps to the database specified as the Referenced databases are used as as a source of data for the auto (Ref. 2, sect. 4.4).]

12. ATTRIBUTE LONG NAME: The long or full attribute name to be used by ALPHA and ALPHAMA-based programs -- The long attribute name
is usually the same as found in the data description file, but the
table provides the means to table provides the means to change the name used by ALPHA and
ALPHAMA users without changing the database. The long 
attribute name may be 25 characters or fewer, and no two names in the same database may be exactly alike.

13. RECORD UID FLAG: Flag used to indicate whether or not the attribute is one of the attributes required to uniquely identify each record:

$$
\begin{aligned}
& 0 . . . . \text { Attribute is not used to uniquely identify } \\
& \text { records in the database } \\
& 1 \ldots . . . \text { Attribute is one of the attributes uniquely } \\
& \text { identifying records in the database }
\end{aligned}
$$

14. FORCED ENTPY FLAG: Flag used to indicate whether default values are to be accepted for that attribute:

$0 \ldots$. ... Default values accepted

1 ..... The user must enter a value for this attribute

15. ATTRIBUTE ARRAY FLAG: ALPHAMA considers an attribute array to be a group of attributes whose names begin with the same alphabetj.c characters followed by a number (e.g., KEY1, KEY2, and KEY10) -- A nonzero value in the master table indicates this attribute is a member of an array. If at any point in ADD mode as the user is entering values for members of the array, he/she inputs only <Enter>, ALPHAMA skips the rest of the members of the array in the ADD list.

16. NO-RETRIEVE-ON-MAP FLAG: Flag used to indicate whether an attribute is to be set from the value in a matching attribute of a reference database (see sect. 3.6.4):

$$
\begin{aligned}
& 0 \ldots \ldots \text { Accept the value } \\
& 1 \ldots \ldots \text { Accept if existing value is blank or zero (0) } \\
& 2 \ldots . . \text { Do not accept the value }
\end{aligned}
$$

\subsection{RE F ERE N C E D D A T A (DBNAME.REF)}

The reference file (DBNAME.REF) is a list of file specifications for one or more support files associated with a database. When you specify a database at the <Database> prompt and its master table (DBNAME.TBL) indicates the existence of a reference file, ALPHAMA looks at it and opens any support files it finds listed there. For example, as indicated by Exhibit 6 , the database BUYER.DMS has a support database file PTS.DMS. Thus the contents of BUYER.REF is the specification U5:[SP4.PTS]PTS.DMS. 


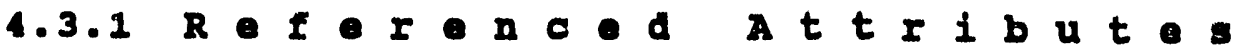

A referenced attribute, designated in DBNAME.TBL, is an index or key to a support database file listed in DBNAME.REF. Each referenced attribute in the main database maps to one support database reference attribute (e.g., a referenced attribute with a DBNAME. TBL map code of 2 tells ALPHAMA to link with the second database listed in DBNAME. REF for a referenced attribute). The automatic updating of matching referenced attributes is described in Sect. 3.6.4

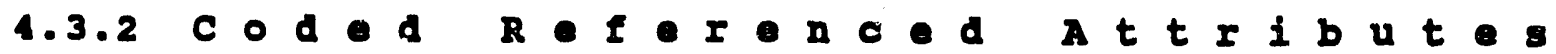

A coded attribute is a type of referenced attribute; it has an assigned code, usually shorter than the name (or phrase) it represents. Either the code or the name can be used to add or edit a referenced attribute. An example of coded data input is in item 31 of the example ADD session (Sect. 5.1) and in item 34 of the example EDIT session (sect. 5.2).

To use a code to add or edit an attribute, (1) the attribute must have a code in the referenced database and (2) the input string should be that code. When input does not exceed the number of charaters allowed for a code but no code actually exists in the referenced database, ALPHAMA does accept the input; however, the program then displays a warning that the code does not exist. When the input string for a code exceeds the number of characters allowed for the code, ALPHAMA discounts the input as a code and instead searches the referenced database for the string.

When a user enters the name (vs code) of a coded attribute, ALPHAMA searches the referenced database for the name -- but stores its associated code as the value of the attribute. If one or more entries exist in the referenced database that contain but do not exactly equal the characters input, ALPHAMA displays those names and their associated codes. ALPHAMA then repeats the prompt for the value of the referenced attribute.

The first attribute of the referenced database must be the code, and the second attribute must be the name. Additional attributes may exist in the referenced database, but the code and name must be the first two attributes. Coded attributes must be listed in the master table (DBNAME.TBL), and they must be defined as text attributes of five characters or fewer.

Inputting a code instead of the full name or phrase represented by that code offers three advantages.

1. Because the code is generally shorter than the name or phrase it represents, the code is faster and easier to input. 
2. Inputting the entire name or phrase may result in attribute values that the computer will not recognize as equal although the user intends them to be. These problems may result from typographical errors, differences in abbreviations, differences in capitalization, differences in word order, etc. Inputting codes therefore provides for greater data integrity and consistency.

3. Using the shorter codes in the main database tends to decrease its size. In turn, a smaller database tends to improve the efficiency of both the maintenance function and user queries to the database. Using coded data usually results in a reduction of the overall disk storage requirements of the application.

\subsection{T E M P I A T E (DBNAME.TEM)}

The values of some attributes require conformity to a certain format or a set of formats. ALPHAMA provides a mechanism that forces short (fewer than 15 characters) text attributes to

conform to a specified format. The template file (DBNAME.TEM) contains the short name of the attribute in columns 1 through 5 and an acceptable template (format) for that attribute beginning in column 6. A given attribute may appear as many times as necessary to list all acceptable formats. Without the existence of a template file, the only restrictions for the value of a short text attribute are that the number of characters input not exceed the maximum number of characters allowed for that attribute and that the value be within any range.

The template description (beginning in column 6 of DBNAME.TEM) uses the following symbols:

N -- A number (0 through 9) must be input in this position.

I -- A letter (A through $Z$ ) must be input in this position.

$X$-- A letter or a number must be input in this position.

$S$-- A space must be input in this position.

" -- The specific character following the quotation mark must be input in this position.

A -- Anything (letter, number, space, or special character) may be input in this position.

A number preceding any of these symbols is considered a multiplier for that symbol; for example, $5 \mathrm{~N}$ is equivilent to NNNNN. 


\subsection{H A N G E L O G (DBNAME.CHA)}

For each record added, changed, or deleted, ALPHAMA records the transaction in a change log (DBNAME.CHA), which is located in the same computer area as the database being maintained. The change $\mathrm{log}$ is automatically created by ALPHAMA if one does not already exist. It contains the record UID and the old and new value of each attribute changed for every record added or changed. The log contains UIDs for deleted records; the date and time; and the group member number (GMN) that initiated the changes. The format of the change $\log$ is in Table 6 below.

\begin{tabular}{|c|c|c|c|c|}
\hline UID & Current value & old value & New & $\operatorname{val1}$ \\
\hline $\begin{array}{l}\text { DD-MM- } \\
\text { ID1 } \\
\text { ID2 } \\
\text { ID3 } \\
\text { ID4 }\end{array}$ & $\begin{array}{ll}\text { YY } & \text { MM:SS } \\
\operatorname{CV}(1) \\
\operatorname{CV}(2) \\
\operatorname{cV}(3) \\
\operatorname{cV}(4)\end{array}$ & [GMN] & & \\
\hline$n+===$ & & & & \\
\hline $\begin{array}{l}\text { ID1 } \\
\text { ID2 } \\
\text { ID3 } \\
\text { ID }(n)\end{array}$ & & $\begin{array}{l}\text { ov (1) } \\
\text { ov (2) } \\
\text { ov (3) } \\
\text { ov (n) }\end{array}$ & & $\begin{array}{l}\text { nv (1) } \\
\text { nv (2) } \\
\text { nv (3) } \\
\text { nv (n) }\end{array}$ \\
\hline
\end{tabular}

Items in the change log (Table 6) are as follows:

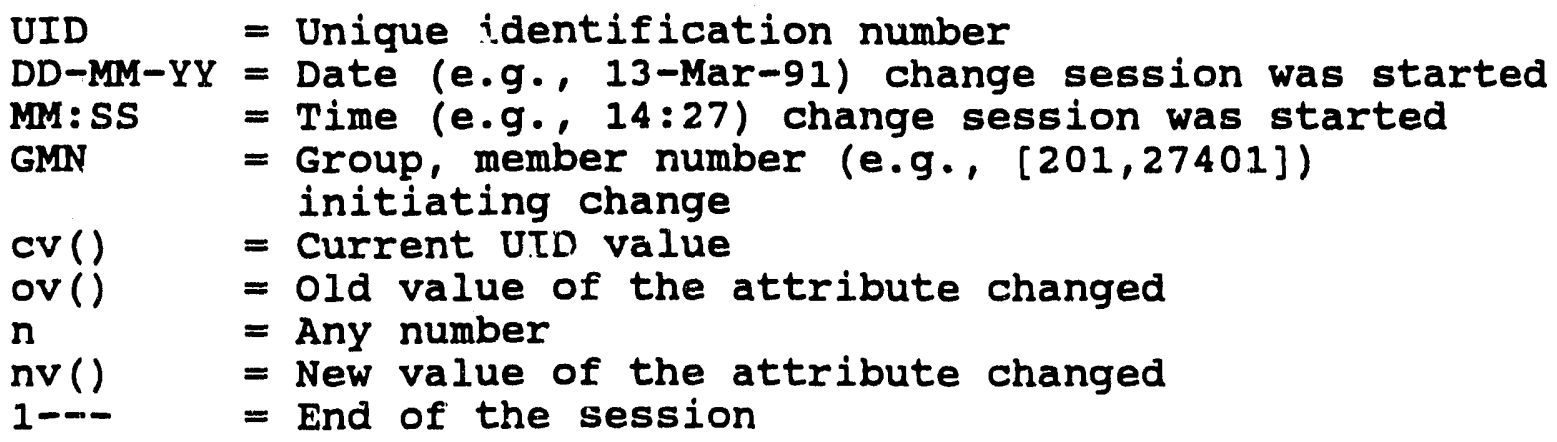

Each change session begins with the date/time/GMN line and ends with the Iine "1-_-". A change session begins with the ADD or EDIT mode and ends when an END, QUIT, or <Enter> is issued. 
The change log may be used with the DBRECOVER program to restore a database that has been lost. DBRECOVER requires the existence of a copy of the database from a system backup and a change log that includes all records from the date of the backup to the date of the restore. The program provides the capability to make all changes recorded in the change log to the backup copy of the database. The change log grows with each addition, correction, or deletion and should be printed and deleted using some appropriate schedule to ensure that the database is okay.

\subsection{A D D I I 8 T (DBNAME.ADD)}

Databases contain attributes, which are required to satisfy the information needs. Usually only a subset of the attributes in the database has values available when a record is added. To enhance the efficiency of adding records, ALPHAMA provides a technique to order the prompting for attributes that have values. These attributes are entered in the DBNAME.ADD file in the order

that the user wishes to be prompted. The order of the ADD file need not correspond to the order of the DBNAME.DMD. Attributes not appearing in the DBNAME.ADD file are not used for prompts during an add session. Attributes not in the ADD list may be added by choosing the EDIT option at the Action? prompt or by editing an existing record. If an $A D D$ list is not available, DBNAME.DMD is used as the effective ADD list.

The ADD file used for the exampje sessions, TSTMA.ADD, is in Appendix B, Sect. B.6.

\subsection{I P I I 8 T (DBWAME. BKP)}

The SKIP list is used in conjunction with the ADD list. Designated by DBNAME.SKP, the SKIP list contains database attributes in the ADD list and in the same order as the attributes in the ADD list. Attributes in the SKIP list denote which attribute in the ADD list the user wishes to "skip" to. After the user exercises the SKIP command, ALPHAMA prompts for the next attribute in the SKIP list that has not been processed. The program skips all attributes between the current location in the ADD list and the entry that matches the next attribute in the SKIP list. (Default values are stored for all attributes skipped.)

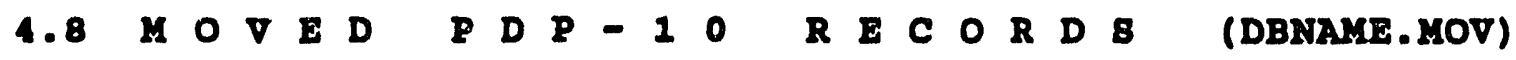

Users on the PDP-10 computer have access to the MOVE command, which, instead of deleting records causes them to be written to a database DBNAME.MOV. This database has exactly the same structure as the database being maintained. For example, if in item 16

(Sect. 5.3) of the example DELETE session, MOVE were 
selected instead of DELETE (see Exhibit 11), the record identified in item 15 of that example session would be written to the database TSTMA.MOV before being deleted from TSTMA.DMS. See Sect. 3.7.10.

\subsection{E R R O R F I I E (DBNAME.ERR)}

The error file (DBNAME.ERR) contains the errors that ALPHAMA is unable to resolve. Errors represented by entries in this file should be investigated and resolved by the person responsible for the application. 


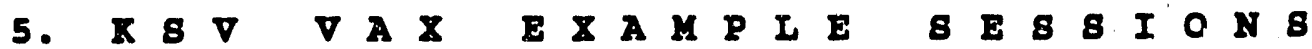

Chapter 5 presents examples (from the KSV VAX computer) of typical ALPHAMA runs that provide an overview of how the program works. Although these runs do not cover all of the program options, they do constitute a representative sample of options avilable for adding (Sect. 5.1), editing (Sect. 5.2), and deleting (Sect. 5.3) records. Each section builds on the one before it; hence, information in sect. 5.2, for example, may have been established in sect. 5.1.

Although reading through the examples can serve as a good review of the program's features, the chapter is designed as a reflection of hands-on exercises. Users are encouraged to copy the example files into their own computer area and work through the commands described in each step. (The files that control the examples are described in Appendix B.)

ALPHAMA is a friendly system, as working through the examples reveal. Anytime you are unsure of what to do next, remember that online Help is always available--just enter one or two question marks, and ALPHAMA will display the next step. Generally new users can learn how to use ALPHAMA to maintain an existing database by simply using the question mark(s) and following ALPHAMA's prompts.

Occasionally examples of keyed input intentionally include typographic and other errors so that subsequent discussions can present editing methods. Also, user input is sometimes displayed here with lowercase characters (vs the usual uppercase convention of this manual). As for the hardcopy you are reading, screen display samples in chap. 5 basically follow the conventions listed in sect. 1.2 except where use of lowercase characters for user input is necessary to describe an ALPHAMA feature. To distinguish each sample from the explanation that follows it, brackets surround commentary. Each of the three sessions begins on a new page.

To begin each session,

1. Log on to the KSV VAX computer (items 1 through 6).

> > 2. Copy the Information Center test database (TSTMA) files into your user area (item 7).

(HINT: Although Chap. 5 examples present short ALPHAMA prompts, you may wish to request long prompting at first -o provide fuller information and thus accelerate the learning process.) 
TO BEGIN A KSV VAX SESSION:

1. <Enter $>\langle$ Enter $\rangle$

[Turn on terminal (hardwired) and depress the <Enter> key a couple of times.]

2. CENTRAL NETWORK HOST

[ This is acknowledgment by Network Control. ]

3. WHICH SYSTEM ( 1 THRU 99)? 61<Enter>

[ System 61 is the KSV VAX computer. ]

4. Username: UID<Enter>

[ Begin log-in procedure by entering your user identification (UID) number. ]

5. Password: PASSWORD<Enter>

[ Enter your unique password (which will not display on the screen. ]

6. Welcome to the KSV VAx system, node KSV2 running

$\mathrm{VAX} / \mathrm{VMS}$ version $5 \mathrm{C}$.

[ Log-in is complete. ]

7. \$ COPY U7: [INF.TESTDB]TSTMA. * [ ]<Enter>

[ At the dollar (\$) prompt, which indicates a successful log-in procedure, copy the test database (TSTMA. DMS) to your own area. (ALPHAMA will automatically copy support files.)

Later, at the end of the session, you can delete the files from your area (DELETE TSTMA.*;*<Enter>). Anytime you want to repeat a session, copy the clean test database file. again. ] 


\subsection{E 8 8 I O N 1: A D D A RE C O R D}

This example session contains an item-by-item discussion of how to add a record to a database. Assume that you maintain database TSTMA.DMS and want to add a new record to it. The data for the new record are listed in Table 7 . The required attributes IDI, ID2, ID3, and ID4 uniquely identify each record in TSTMA.DMS. (NOTE: Only the values of the attributes that uniquely identify each record must be supplied to add or edit a record using ALPHAMA.) Attributes TEXT2 and TEXT3 exist in TSTMA.DMS but do not require values for the record to be added.

TABLE 7. Data for the Example New Record

$\begin{array}{ll}\text { AD1 } & \text { z21234567 } \\ \text { ID2 } & \text { new1 } \\ \text { ID3 } & 12 \\ \text { ID4 } & \text { "Short text att" } \\ \text { TEXT1 } & \text { This is an example session useing the ALPHAMA } \\ \text { TEXT4 } & \text { program to add a record to the database. The } \\ & \text { database and all nicessary support files are } \\ \text { INT1 } & \text { assumed to exist. } \\ \text { REAL1 } & 123,000 \\ \text { REAL2 } & 234.567 \\ \text { EF1 } & 3,456.7 \\ \text { EF2 } & 234.56 \mathrm{E}+12 \\ \text { DN1 } & 00345 \mathrm{E}-3 \\ \text { AD } & \text { December } 16,1991 \\ \end{array}$

You can begin the session once you have logged on to the KSV VAX computer and have copied the test database to your user area (Sect. 5, items 1 through 7). When you reach the system prompt, issue the command to run ALPHAMA (item 8). Then follow the subsequent items, which represent user input and ALPHAMA responses.

\section{8. \$ ALPHAMA $<$ Enter $>$}

[ This is the input command to run ALPHAMA module; it causes system logical ALPHAMA to execute the operational version of ALPHAMA. ] 
9. ALPHAMA Maintenance Program - Version : V5C

Date: $12 / 31 / 91$

Time: $09: 49$

[ The system (monitor) passes control to ALPHAMA. From this point, ALPHAMA processes input; system-level commands are not valid. They will not be until you exit from the ALPHAMA program. Before further execution of ALPHAMA, you may see an ALPHAMA FORCED MESSAGE, which informs users of current enhancements and upgrades to tha ALPHAMA system as well as of contacts for any problems that might be encountered while executing ALPHAMA. ]

10. Display System Hotnews $(\mathrm{Y} / \mathrm{N})$ ? $\mathrm{Y}<$ Enter $>$

This is the System 1032 version of the ALPHAMA Maintenance Program. ALPHAMA operates much like the ALPIA query program.

- One of the new features included here is IAST (LA): an option available when you are adding a new record. It instructs ALPHAMA to use for the new record the default values from the previous (last) record added or edited instead of those from the master table. This is useful if you are adding a series of records that are similar or identical except for their unique identifiers. When ALPHAMA prompts for whether you wish to add a record, enter LAST (or LA) instead of YES.

[ ALPHAMA prompts users about viewing the ALPHAMA System Hotnews, which gives detailed information on enhancements, upgrades, and/or new features to the ALPHAMA system. It appears only when a message to the users is needed. An answer of yes, indicated with the letter "Y" ( $Y$ ) causes ALPHAMA to list the latest Hotnews items. A response of no with the letter "N" (N) or <Enter> (default) causes ALPHAMA go directly to the <Database> prompt. ]

11. <Database> TSTMA<Enter>

.... Opening database TSTMA

[ First, ALPHAMA requires the file specification for the database to be maintained. A valid response can be the filecode of a database listed on an index or the full specification, in the format:

<Database> DEV:[DIRECTORY] FILENAME. EXT<Enter> 
See sect. 3.4 and Exhibit 6 for a discussion of using the Database (DB) command and the input default. Second, ALPHAMA searches for the support files (DBNAME.TBL, DBNAME.REF, DBNAME.TEM, and DBNAME.ADD). ALPHAMA loads the information from the support files and opens the database. (If a user does not have access to maintain the database, ALPHAMA displays an error message and repeats its request for a database specification.) ]

12. LONG prompting $(\mathrm{Y} / \mathrm{N}) \quad \mathrm{N}<$ Enter $>$

[ Select full attribute names or abbreviations as prompts. A yes $(Y)$ response causes ALPHAMA to prompt with the full attribute name. A response of no (N) or <Enter> (default) results in the use of the abbreviation. The abbreviations and names reside in DBNAME.TBL. If no DBNAME. TBL exists, use the names and abbreviations in the DBNAME.DMD file. The example session uses short prompts. ]

13. Do you want to see default values displayed? (Y/N) N<Enter $>$

[A yes ( $Y$ ) answer causes ALPHAMA to display any default values as they are applied to attributes during a record Add. If you do not wish to view the default values, type the letter "N" (N). ]

14. 7 records available

[ ALPHAMA reports the number of active records in the database. ]

15. Search for:

[ ALPHAMA begins the process of requesting the identifying attributes of a record. If no DBNAME. TBL file exists or if the unique identifiers are not indicated in the DBNAME.TBL file, ALPHAMA requires inputting the attributes that uniquely identify each record: (see sect. 3.5.1). ALPHAMA remembers the UIDs for this example session. ]

16. ID1? z2123m567<Enter>

ID1 should have been entered

in the following format: xxnnnnnnx

Input was received as follows : ZZ123M567

Errors were detected in the following position: 
Format is : $n$ text number

$: 1$ letter
$: x$ text number or letter
: a any character

[ ALPHAMA requests input for the value of the first attribute that serves as a unique identifier (ID1). When building a database, select attribute names and abbreviations that best describe each attribute. ID1 is appropriate because TSTMA.DMS is used only for testing and example purposes. (See

Appendix B, sects. B.1 and B.2, for the abbreviations, names, and constraints of the attributes in TSTMA.DMS.)

In this example session, the value for IDI should be zz1234567 (Table 7). Thus the input zz123m567 is incorrect. Here ALPHAMA informs the user that the input contains an error. The letter " $M$ " in the input does not conform to any of the allowed templates (all of which are listed in TSTMA.TEM, Appendix B, Sect. B.4) defined in DBNAME.TEM. ALPHAMA displays an error message that "ZZ123M567" is not an acceptable value for IDI: It marks the character(s) that violate the template with a caret (^) -in this case, the letter "M". ]

(NOTE: The screen response is "Zz123M567" rather than the "zz123m567" because ALPHAMA automatically converts all short text attribute values to uppercase.)

17. ID1? zz1234567<Enter>

[ Inputting an unacceptable value causes ALPHAMA to ask for an acceptable value. The user then inputs the corrected value (zz1234567) for ID1, and ALPHAMA checks to see whether this value is in an acceptable format. ]

18. No IDI $=\mathrm{zz} 1234567$

Add ? Y<Enter $>$

[ ALPHAMA does not find the input value for UID "ID1" and offers the user the opportunity to add the record. A yes response $(Y)$ takes ALPHAMA to the ADD mode, where it prompts for the remaining attributes that uniquely identify a record, in accordance with the DBNAME.ADD file. If no DBNAME.ADD file exists, ALPHAMA requests the remaining attributes in order of DBNAME.DMD. ] 
19. ID2? new $1<$ Enter>

ID3? $12<$ Enter>

ID4? 1<Enter>

[ ALPHAMA requests the remaining unique attribute (UID) values. ID2, a short text attribute, converts the value "new1" to uppercase "NEW1"; ALPHAMA checks the unique identifier values for acceptable format. ]

20. TEXT1? "Short text att"<Enter>

[ TEXT1 is 14 characters. Any value beyond 14 characters will result in the message: "A maximum of 14 characters may be entered. Truncation will occur. Do you wish to reenter? $(Y / N)$." If the user enters the letter "Y" here, ALPHAMA reprompts with "TEXT1?" -- because pressing <Enter> will cause only the first 14 characters to be stored.

spaces and commas are included as part of a value and require the use of quotation marks around the entire value. If no default value is specified in the DBNAME.TBL, blanks are stored in response to <Enter>. ]

21. TEXT2>

$>$ <Enter>

[ Pressing <Enter> accepts the default value for TEXT2. If no default value is specified in the DBNAME.TBL file, ALPHAMA stores blanks in response to <Enter>. ]

22. TEXT4 >

$>$ this is an example session useing the ALPHAMA program $t$ - add a record to the database. The database and all nicessa ry support files are assumed to exist.<Enter>

[ Note that ALPHAMA does not prompt for TEXT3, which is in TSTMA.DMD but not included in the TSTMA. ADD list. Although TSTMA.ADD may or may not follow the same order as TSTMA.DMD, TSTMA.ADD determines the attributes requested in ADD mode and the order in which they are requested.

Although the example input for the long text attribute TEXT4 is three lines long, ALPHAMA requires an <Enter> only at the end of the text - not at the end of each line. Here the user presses <Enter> and initiates the store. If the number of characters input exceeds the number allowed, ALPHAMA stores the maximum number and truncates the rest. 
(Note that the display input wraps lines without regard to words; thus "to" and "nicessary" break across two lines.) The example input includes two misspelled words: "useing" and "nicessary" (cf. Table 7). Correction of these errors takes place in the example EDIT session, sect. 5.2. ]

23. INT1? $823000<$ Enter $>$

ERROR - 823000 is greater than hi-range value of 250000

[ The number 823000 exceeds the range aliowed for INTI;

ALPHAMA informs the user of the range limit violation. The range specification in TSTMA.TBL requires that an entry for IN'I not exceed 250000; therefore, ALPHAMA rejects the value 823000 .

WARNING: Commas and spaces indicate stringed input. Input of 823,000 or 823000 results in INTI $=823$ and REALI $=0.0$; thus the next prompt would be for REAL2 and not REAL1. ]

24. INT1? $123000<$ Enter $>$

[ Input request for INT1 is repeated (note that the response of 123000 does not have a comma after the 3). ]

\section{REAL1? $1234.567<$ Enter>}

REAL2? 3456.7<Enter>

[ The user inputs the values for the next two attributes; real number attributes may have decimal points but not commas or spaces.

REALI $=1234.567$ is not the correct value (cf. Table 7 , where REAL1 $=234.567)$; however, it does not violate any range or template and is therefore accepted. (See item 33 for how to correct the entry.)

Real attributes may contain a sign (+ or -), numbers (0-9), and decimal point only. Commas are delimiters and may not be used. ]

\section{6. $\mathrm{AD}$ ? RICHMOND<Enter > \}}

( ALPHAMA prompts for the value of the attribute AD (Associate Director), and the user enters the name (RICHMOND). Instead of the name the user could have entered the attribute code ( 3 ), because $A D$ is a coded attribute. 
The coded attribute AD resides in TSTMA.TBL. ALPHAMA searches the reference file (see Appendix B., Sect. B.3, for an example of a reference file) for the input value. Because $A D$ is defined in TSTMA.TBL as a coded (mapped) one-character text attribute, ALPHAMA searches the second attribute in the referenced database GUIDE DIR GENERAL:ADNA.DMS (Appendix B, Sect. B.3) for the value "RICHMOND." ALPHAMA accepts either the code "3" or the name "RICHMOND" as input. Code "3" is stored as the value of the attribute. ]

27. EF1? 234.56E12<Enter>

EF2? - . $00345 e-3<$ Enter>

[ ALPHAMA processes numbers expressed in scientific notation (exponential format). Exponential format attributes may contain a sign ( or - ) for the number, numbers $(0-9)$, a decimal point for the number, the letter " $E$ " in either uppercase or lowercase, a sign $(+$ or -$)$ for the exponent, and no more than two numbers $(0-9)$ for the exponent. ]

\section{DN1? 121691<Enter>}

[ ALPHAMA accepts many date formats. The most unambiguous date formats are MMDDYY, MM/DD/YY, and MM-DD-YY

(sect. 2.2.1.2). ALPHAMA generally outputs dates as MM/DD/YYYY. When setting up a database, always define dates as having at least six characters. ]

29. Action? ?<Enter>

Must be one of:

ABORT ADD <Enter> DISPLAY EDIT END QUIT

[ ALPHAMA asks what to do with the record. The user input of a single question mark (?) causes ALPHAMA to give a brief list of the available commands. The Action? prompt indicates all attributes in the ADD list are ready to be processed. Each of these options is discussed in detail in other sections of this manual.

NOTE: If at any point uncertainties arise about the available option or required response, ALPHAMA provides assistance when the user types one or two question marks (? or ??). In response to one question mark (?), ALPHAMA displays short HELP messages. In response to a two (??), ALPHAMA displays a more extensive HELP response. ] 
30. Action? EDIT<Enter>

[ EDIT allows corrections to mistakes before adding the record to the database. The value of attribute $A D$ is suspect, and REALI is incorrect. They must be edited before adding the record. (Spelling errors are corrected in the example of sect. 5.2). ]

31. EDIT? AD<Enter>

$A D=3$

? <Enter>

[ To verify the value stored in the attribute $A D$, the user enters the attribute abbreviation. ALPHAMA displays the code stored in $A D$ and asks for the new value. The <Enter> tells ALPHAMA to retain the current value and return to the EDIT? prompt. (Note that ALPHAMA stored "3" as the value of AD even though the name "RICHMOND" and not the code was entered.) ]

32. EDIT? REAL1<Enter>

REALI $=1234.57$

? $\quad 234.567<$ Enter>

[ The value for REALl (entered in item 25) was incorrect, so here the user edits the entry. At the EDIT? prompt, the user types the name of the attribute. ALPHAMA displays the current (incorrect) value and then awaits input of the new value: 234.567 . When the user types this (234.567), ALPHAMA records the new value of REALl and redisplays the EDIT? prompt.

(NOTE: Attributes can be edited in any order. Display format is determined by the DBNAME.TBL file. Here, for example, the format specified for REAL1 in TSTMA.TBL (see Appendix B, Sect. B.2) is F9.2; thus REAL1 is displayed rounded to two digits right of the decimal (1234.57) even though input for item 25 was three digits after the decimal (1234.567). The actual value stored in the database depends on how the attribute is defined in DBNAME.DMD. ]

\section{EDIT? END<Enter $>$}

[ ALPHAMA prompts for another attribute to edit. A response of END or <Enter> indicates the edit of this record is complete. ] 
34. Action? ADD<Enter $>$

[ ALPHAMA adds the record to the database, exits ADD mode, and returns to search for: prompt. Processing of the record is complete. ]

35. Search for:

ID1? END<Enter>

[ ALPHAMA asks for the next record to be added or edited. Input of END or QUIT indicates no more records to add or edit at this time. END returns the user to the <Database> prompt (QUIT would have exited ALPHAMA). ]

36. <Database> END<Enter>

[ALPHAMA permits maintenance of another database. The response END indicates no other database maintenance at this time and ends the session. ]

37. Bye..

[ ALPHAMA execution ends; ALPHAMA returns to the system prompt. ] 


\subsection{E E B I O N $2:$ E D I T A R E C O R D}

This example session shows how to edit a record; it assumes that you have already completed the Add exercises in Sect. 5.1. As in Sect. 5.1, the sample data are from the test database (altered by input made in sect. 5.1); ID1, ID2, ID3, and ID4 uniquely identify database attributes. After editing, the attribute values of the record in the test database should be those listed in Table 8. See Appendix $B$ for a description of the example support files. (NOTE: An initial exposure to EDIT mode is found in items 30 through 33 of the example ADD sessior; sect. 5.1.)

TABLE 8. Opdated Attribute Values of the Example Edited Record
Attribute

This example session assumes that you have logged on to the KSV VAX computer (sect. 5, items 1 through 6), copied the test database into your user area (Sect. 5 , item 7), and performed the example items in sect. 5.1. Once at the system prompt (\$), issue the command to run ALPHAMA (item 8 ).

\section{8. \$ ALPHAMA<Enter>}

[ This is the input command to run ALPHAMA module. The dollar sign (\$) for the VAX computer and a period (.) for the PDP-10 computer indicates a successful log-on. Entering

ALPHAMA<Enter> causes the system logical, ALPHAMA, to execute the operational version of ALPHAMA. ]

9. ALPHAMA Maintenance Program - Version : V5C

Date: $12 / 31 / 91$

Time: $09: 49$ 
10. Display System Hotnews $(Y / N) ? N<$ Enter $>$

[ This prompt may or may not appear, depending on whether information is available. ]

11. <Database> TSTMA.DMS<Enter> .... Opening database TSTMA

[ ALPHAMA loads the information from the support files and opens the database.

If the user does not have access to maintain the specified database, ALPHAMA displays an error message and repeats the request for a database specification. ]

12. LONG prompting $(\mathrm{Y} / \mathrm{N})$ <Enter>

[ Default is short prompting; <Enter> means no. ]

13. Do you want to see default values displayed? $(Y / N)$ <Enter>

[ Default is not to display default values.]

14. 8 records available

[ ALPHAMA reports the number of active records in the database. From this point, ALPHAMA begins the process of requesting the identifying attributes of a record. ]

15. Search for:

ID1? z21234567<Enter>

ID2? VALUE<Enter>

ID2 1 record found

NEW1

ID2? NEW1<Enter>

ID3? VALUE<Enter>

ID3 1 record found

12

ID3? $12<$ Enter>

ID4? VALUE<Enter>

ID4 1 record found

$$
1
$$

ID4? 1<Enter>

[ Before editing any record, the exact record must be identified; thus here the user inputs the UID for an existing record. If the input UID does not match an existing record, 
ALPHAMA permits adding the record. If a record UID matches an existing record (as in this example), the EDIT mode is automatically invoked. One and only ONE RECORD CAN BE EDITED AT A TIME.

In this example the user does not remember all the values input for the UIDs of the record to be edited. Therefore, the user recalis the value for the first UID and then issues the Value command, which asks ALPHAMA to display the values of subsequent UIDs. ]

16. EDIT? DISPLAY<Enter>

ID1 $=$ ZZ1234567 ID2 = NEW1

ID3 $=12$ ID4 $=1 \quad$ TEXT1 = short text att

TEXT2 =

$\operatorname{TEXT3}=$

TEXT4 = This is an example session useing the ALPHAMA program to add a record to the database. The database and all

INT1 $1=123000$ nicessary support files are assumed to exist.

REALI $=234.57$

REAL2 $=3456.70$

$A D=3$

$E F I=.234560000 \mathrm{E}+15$

EF2. $=-.34500000 \mathrm{E}-05$

DN1 $=12 / 16 / 1991$

[ EDIT? prompts for the attribute to edit. DISPIAY tells ALPHAMA to display the current contents of the record selected.

One of the most useful options available at the EDIT? prompt is DISPLAY, which causes ALPHAMA to display the current values of all attributes for the record being edited. The values shown for several attributes are worth noting. Attributes ID2 and TEXT1 are both short text attributes (defined as 15 characters or fewer). Although ID2 was entered as "new1" (without the quotation marks) and TEXT1 as "Short text att" (with quotation marks), the former appears in uppercase. To promote consistency, ALPHAMA converts all lowercase letters to uppercase characters for text attributes unless they are enclosed in quotation marks - as "Short text att" was, to account for the spaces.

The attribute TEXT4, is defined as more than 15 characters in TSTMA.DMD and thus is a long text attribute. Therefore, its value is stored using uppercase and lowercase characters.

(NOTE: Although the input words "to" and "nicessary" are broken across two lines (sect. 5.1, item ?2), here they are not broken: they are in "one piece.") 
The attribute REAL1 displayed as "234.57" was input as 234.567 (Sect. 5.1, item 25). However, the output format for REALI specified by TSTMA.TBL (Apnendix B, Sect. B.2) allows for only two significant digits to the right of the decimal. Therefore, input for REALI was rounded to comply with the format specifications. The attribute REAL2 input as 3456.7 (see sect. 5.1, item 25) is displayed as "3456.70" to comply with format specifications.

The attribute $A D$ is displayed as " 3 " -- the code for "RICHMOND" (see sect. 5.1, item 31).

Attributes that accept scientific notation are displayed in uniform format. EF1, originally input as 234.56E12 (see sect. 5.1, item 27), is displayed as ".234560000E15"; EF2, originally input as $-.00345 \mathrm{e}-3$ (see sect. 5.1, item 27), is displayed as "-. $\$ 400000 \mathrm{E}-05 "$ instead.

Date definition in the DBNAME.DMD and DBNAME.TBL files requires at least eight characters to display the complete date. Note that DN1 entered as 121691 (see sect. 5.1, item 28) is displayed as $12 / 16 / 1991$. ]

\section{EDIT? TEXT2<Enter>}

[ ALPHAMA asks which attribute to edit next; the user specifies TEXT2.

Many options are available at the EDIT? prompt; inputting one or two question marks (? or ??) causes AIPHAMA to list the available options. The most common response is the attribute to edit. ALPHAMA displays the current value of the attribute and permits changing its value. ]

19. ?No text to edit. Would you like to add some? Y<Enter> $>$ Somename AM<Enter>

[ TEXT2 contains no text; user adds text for attribute TEXT2.

originally only an <Enter> was input for the value of TEXT2 (Sect. 5.1, item 21). TEXT2 accepts text data in many forms. NOTE: The user must choose a format for text attributes and consistently input all text in that format. consistent format application results in more efficient searches on text attributes using the ALPHA or ALPHAMA user program. ] 
19. EDIT? TEXT3<Enter>

?no text to edit. Would you like to add some? Y<Enter>

$>$ This attribute was not in the ADD list. <Enter>

[ This input adds text to the attribute TEXT3. TEXT3 was not in the $A D D$ list and thus not entered during the ADD session. ]

20. EDIT? TEXT4<Enter>

[ The user requests to edit attribute TEXT4. The attribute TEXT4 allows up to 600 characters. ALPHAMA provides some very useful capabilities for editing long text attributes. Many of these capabilities are demonstrated and discussed in items 21 through 29. ALPHAMA displays a list of these capabilitise in response to user input of one or two question marks (? or ??) at the ">" prompt. ]

21. This is an example session useing the ALPHAMA program to add a record

[ ALPHAMA displays the first line of text and goes into the long text EDIT mode. Iine length for this line of text is determined by the computer/terminal screen width. The displayed text does not contain words broken across lines. For example, if all of the characters of the word "program" do not fit on the line of text selected, ALPHAMA will not print any of the characters of "program"; instead the line displayed will end with the word "ALPHAMA". ]

22. > r/useing/using/<Enter>

[ This input is to correct the spelling of the word "useing" in item 21 by replacement. (Reminder: The replacement command is "r"; the delimiter here is "/".)

The prompt ">" indicates that ALPHAMA allows editing the line of text just displayed in item 21. The "r" indicates the replacement of the string "useing" with the string "using". The form of the REPLACE command follows:

R(delimit)CURRENT_STRING (delimit) NEW_STRING (delimiter) <Enter>

The delimiter may be any single character. ALPHAMA uses the character immediately following the "R" as the delimiter for the REPLACE command. Do not use a character that appears in either the "current string" or the "new string" as the delimiter. The slash (/) is used as the delimiter in items 22, 26, and 27. ALPHAMA performs the replacement and displays the corrected line of text. ] 
23. This is an example session using the ALPHAMA program to add a record

$>$ <Enter>

to the database. The database and all nicessary support

files are

$>$ <Enter>

assumed to exist.

$>$ <Enter>

[ The user presses <Enter> twice to move to subsequent

ines of text for viewing and then again to return to the EDIT? prompt. ]

24. EDIT? TEXT4<Enter>

This is an example session using the ALPHAMA program to add a record

$>$ d<Enter $>$

[ The next portion of the text for attribute TEXT4 should say "edit an existing record." Instead of using REPLACE to edit the line, this example uses the DELITE and INSERT options. Inputting the letter "D" (or "d") at the prompt causes the deletion of the line of text displayed. ]

25. > i This is an example session using the ALPHAMA program to edit an existing record. <Enter>

[ The user types in an " $i$ " for insert and types the data. The new line is inserted in front of the previous one. ]

26. to the database. The database and all nicessary support files are

$>r /$ to the database.//

[ This input uses the REPLACE ( $r$ ) command to replace the phrase "to the database." as delimited with slashes. Unlike the use of REPLACE in item 22 , however, the input provides no replacement phrase; without a replacement string, the second slash after the phrase tells ALPHAMA that no string replaces the phrase. In effect, REPLACE ( $r$ ) deletes the phrase. (See Sect. 3.7.2.8.) ]

27. The database and all nicessary support files are $>r / n i / n e /<$ Enter $>$

[ This is the input to correct the spelling of the word "nicessary": REPLACE corrects the spelling. Note that the eitire string "necessary" did not have to be entered, only erough of the string to uniquely identify the characters to be replaced. ] 
28. files are assumed to exist.

$>$ a ALPHAMA is easy to learn. <Enter>

[ Pressing <A> (APPEND option) instructs ALPHAMA to append text to the end of the text currently contained in the attribute. Thus here the text "ALPHAMA ... learn." is appended to the end of the existing contents of TEXT4. ]

29. files are assumed to exist. ALPHAMA is easy to learn. $>$ END<Enter $>$

[ Edit of attribute TEXT4 is completed. The prompt ">" allows further editing. END tells ALPHAMA that editing the attribute is finished. If the attribute is only one display line or the last line of the attribute is displayed, <Enter> is equivalent to END. Inputting just <Enter> at this point in the example produces the same result as inputting END. ]

30. EDIT? INT1<Enter>

INT $1=123000$

? $5678<$ Enter>

[ This input changes the integer attribute INTI from 123000 to 5678. ALPHAMA displays the current value (123000) and accepts the input 5678 as the new value. Remember that commas are not allowed in numeric data; that is, inputting the new value as 5,678 is NOT acceptable; ALPHAMA would consider it stringed input for two attributes (separated by a comma), not one, and display an error message for the second attribute. ]

31. EDIT? REAL2<Enter>

REAL2 $=3456.70$

? $0<$ Enter $>$

[ This input changes the value of REAL2 from 3456.70 to zero $(0.0)$. ALPHAMA displays the current value of 3456.70 and accepts user input of zero (0) as the new value. Remember that inputting only an <Enter> for the new value causes retention of the old value $(3456.70)$. (Another way to do this is with the \# command as described in sect. 3.7.2.2.) ]

32. EDIT? DN1<Enter>

$\mathrm{DN} 1=12 / 16 / 1991$

? jan-20-92<Enter $>$

[ Here DN1 is changed from December 16, 1991 to 1/20/92. ALPHAMA displays the current value (12/16/1991 and accepts the input jan-20-92 as the new value. ] 
33. EDIT? DN1<Enter>

$\mathrm{DN} 1=1 / 20 / 1992$

? <Enter>

[ ALPHAMA displays the value of DN1 for verification and asks for the new value. The user input <Enter> causes the old (current) value $1 / 20 / 92$ to be retained. Note that although ALPHAMA accepts dates in different formats, it always stores them in the format MM/DD/YYYY. ]

34. EDIT? $A D<$ Enter $>$

$A D=3$

? FULKER<Enter>

Did you mean:

Fulkerson

$\mathrm{AD}=3$ EDIT?

? 5 <Enter>

[ The attribute $A D$ is a referenced (coded) attribute, and the referenced file associated with $A D$ is the database U1: [GUIDE DIR GENERAL] ADNA.DMS (Appendix B, Sect. B.3). Here ALPHAMA did not $f$ ind the value FULKER in the referenced database so it displays the closest match it found and asks if the displayed value were the intended input; then ALPHAMA prompts the user for the new value. In this case, the user confirms the change from 3 (RICHMOND) to 5 (FULKERSON).

[For Help on a response, enter one or two question marks (? or ??).] ]

\section{EDIT? DISPLAY<Enter $>$}

$$
\begin{aligned}
\text { ID1 }= & 221234567 \text { ID2 }=\text { NEW1 } \\
\text { ID3 }= & 12 \text { ID4 } 1 \text { TEXT1 = short text att } \\
\text { TEXT2 = } & \text { Somename AM } \\
\text { TEXT3 = This attribute was not in the ADD list. } & \text { TEXT4= } \text { This is an example session using the ALPHAMA program } \\
& \text { to edit an existing record. The database and all } \\
& \text { necessary support files are assumed to exist. ALPHAMA } \\
& \text { is easy to learn. } \\
\text { INT1 = } & 5678 \\
\text { REAL1 = } & 234.57 \\
\text { REAL2 = } & 0.00 \\
\text { AD }= & 5 \\
\text { EF1 }= & .234560000 E+15 \\
\text { EF2 }= & -.34500000 E-05 \\
\text { DN1 }= & 1 / 20 / 1992
\end{aligned}
$$

[ ALPHAMA displays the revised contents of the record.] 
36. EDIT? END<Enter>

[ Revisions to this record are complete. ALPHAMA stores the record and returns to SEARCH mode (Search for: prompt). ]

37. Search for:

IDI? QUIT<Enter>

Bye..

[ No other records are added or edited at this time; ALPHAMA is exited. ALPHAMA closes all files and returns to the system prompt. ] 


\subsection{E 8 B I O N 3: DEIETE A RECOR D}

This example session shows how to delete an unneeded record using the DELETE option of EDIT mode. As in sects. 5.1 and 5.2 , the sample data originated from the test database TSTMA.DMS (support files are described in Appendix B); however, the data have been altered according to the exercises in the preceding sections. Unique identifiers (UIDs) of the record to be deleted are

$$
\begin{aligned}
& \text { ID1 }=\mathrm{ZZ1234567} \\
& \text { ID2 }=\mathrm{NEW1} \\
& \text { ID3 }=12 \\
& \text { ID2.DMS4 = } 1
\end{aligned}
$$

To begin the delete session, log on to the KSV VAX computer (Sect. 5, items 1 through 6) and copy the test database to your user area (Sect. 5, item 7). Then because the DELETE option is a function of EDIT mode (Sect. 3.7.9), continue by following the steps of items 8 through 14 of the EDIT session (sect. 5.2). When you reach the search for: prompt, proceed with item 15 below.

\section{Search for:}

ID1? zz1234567<Enter>

ID2? NEW1<Enter>

ID3? $12<$ Enter $>$

ID4? 1<Enter>

1 record found.

[ Input the record UID for an existing record. ]

\section{EDIT? DELETE<Enter>}

[ Input instructs ALPHAMA to delete the record selected. The record is marked in the database as a deleted record, which is thus not available to ALPHAMA users. However, a deleted record is available via system 1022 and 1032 (DFIND ALL) until a database dump and reload. (DUMP and LOAD are System 1022 and 1032 commands). ]

17. Are you SURE you want to DELETE this record? Y<Enter> Record is DELETED from database

[ ALPHAMA requires confirmation of the user's desire to delete the record before actually doing so, because ALPHAMA users do not have access to deleted records. Any response other than the letters "Y", "YE", or "YES" causes ALPHAMA to ignore the command to delete the record. ] 
18. Search for:

ID1? QUIT<Enter>

Bye. .

[ After marking the previous record as deleted, ALPHAMA requests the attributes that uniquely identify the next record to process. When you have no other records to add or edit, input QUIT to exit ALPHAMA. It indicates no more activity, and ALPHAMA closes all files and returns to the system prompt. ] 


\section{6. $R$ E F E R E N C E}

1. J. W. Grubb, J. K. Lovin, and R. I. Haese, ORNL ALPHA MIS Data Base Manual, ORNL/TM-8655, Union Carbide Corporation, Oak Ridge Natl. Lab., January 1984.

Description of how to design and set up a database and support files utilized by ALPHA; stored on an Energy Systems mainframe as UO: [RDH. MANUALS] TM8655.DOC

2. J. K. Lovin et al., ALPHA MIS User's Manual, rev., ORNL/TM-8621/R1, August 1987, Martin Marietta Energy Systems, Inc., Oak Ridge Nat1. Lab.; revision, ALPHA MIS Reference Manual, ORNL/TM-8621/R2, Martin Marietta Energy Systems, Inc., Oak Ridge Natl. Lab., in press.

Commands and options for ALPHA management information system; stored on an Energy Systems mainframe as UO: [RDH. MANUALS] TM8621.DOC

3. J. K. Lovin et al., ALPHA Tutorial, ORNL/TM-11732, Martin Marietta Energy Systems, Inc., Oak Ridge Natl. Lab., in press.

Self-paced disk tutorial for novice and intermediate ALPHA users; available by e-mail request to HUGHESSE

4. J. W. Grubb et al., ORNL ALPHAMA MIS Programmer's Manual, ORNL/TM-8923, Union Carbide Corporation, Oak Ridge Natl. Lab., May 1984 .

ALPHAMA documentation for programmers (not available to general users)

5. System 1022 User's Reference Manual, version 121A, CompuServe Data Technologies, Cambridge, Mass., n.d. (previous version, 1988).

Narrative guide to system 1022, a management information system that runs on a Digital Equipment Corporation PDP-10 computer; available through the Energy systems online ordering system DO MANUAL (S1022/USR: \$34.16)

6. System 1032 User's Guide, version 8, Compuserve Data Technologies, Cambridge, Mass., n.d. (version 7, 1989).

Nontechnical narrative guide (by topic) to system 1032, a management information system that runs on a Digital Equipment Corporation VAX computer; available through the Energy systems online ordering system DO MANUAL (S1032/USR/V8: $\$ 89.43$ ) 
This page intentionally left blank. 


\section{A P P E D I X A. C O M A N D B: I O G I C A I VIEW O F A T Y P I C A I B E B B I O N}

\section{A.1 GENERAI C O M A N D}

Six general command options (Table A.1) are available at all prompts. The "top-down" logical view of a typical ALPHAMA session presented in Table A.2 lists the prompts and the valid commands and options available at each point in the first part of the SEARCH mode.

\section{TABLE A.1. General Command Options}

$\begin{array}{ll}\text { ?? } & \text { Provides second level of (context-sensitive) Help* } \\ \text { <Enter> } & \text { Mrovides third level of (context-sensitive) Help } \\ & \text { default values if adding; assumes "NO" at prompts } \\ & \text { requesting a "YES" or "No" response } \\ \text { END } & \text { Ends (ceases) current activity; returns to previous } \\ \text { HELP } & \text { Provides fourth level of online Help: detailed } \\ & \text { information on a requested subject -- for example, } \\ & \text { "HELP END" requests information regarding the END } \\ & \text { command (HELP can be used with attributes and } \\ & \text { commands) } \\ \text { QUIT } & \text { Terminates the session and returns user to } \\ & \text { the system prompt }\end{array}$

*First-level Help (prompts and error messages) displays without user request. 
TABLE A.2. Commands and Options in BEARCH Mode

$\begin{array}{ll}\text { Display prompt } & \text { Description of command or option } \\ \text { and valid responses }\end{array}$


A.2 E D I T MODE

If a record were found in the SEARCH mode, the next options would be those listed in Table A.3.

TABLE A.3. Commands and Options in EDIT Kode when

a Record Has Been Found

Input option

or command
Description of command or option and valid responses

EDIT?

ABORT

APPLY

Att

<\#>

<Enter >

Value

END

QUIT

DELETE

DISPIAY

MOVE

SAVE

SKIP Att
- Cancel all changes made to the record; return to search for: prompt

- Make changes to record as per the SAVE command

- Find any valid attribute name or abbreviation input by the user (Att); display the current value, with the following options available:

- Delete current value and set to or blanks or zero

- Keep current value; return to EDIT? prompt

- Set a new attribute value of $x \times x$

- store changes; return to search for:

- Store changes; return to system prompt

- Delete record from database

- Display current values for all attributes

- Remove record from database and place it in a System 1022 database with the same name as the selected database (but with file extension MOV) and in the same user area (valid only on the PDP-10 computer)

- Save (store) preceding changes for application (see APPLY) to subsequent records

- Using the ADD list, begin prompting for attributes starting with user-specified Att (attribute), which must be in the ADD list and not be a unique identifer 


\section{A. 3 A D D M O D E}

If the record were not found, ALPHAMA would display that "No attribute = value entered" and would prompt: Add? The response commands listed in Table A.4 would be appropriate.

\section{TABLE A.4. Commands and Options in SEARCE Mode when a Record Has Not Been Found}

Input option or command
Description of command or option and valid responses

Add?
ABORT
- Return to Search for: prompt
ILAST
- Use the last record added or edited as the source of any defaults (otherwise, same as yes)
No
- Do not enter ADD mode; go back to Search for: prompt
YES
- Begin prompting for remaining attributes in $A D D$ list (i.e., go into ADD mode)


If the user elects to add a record, ALPHAMA prompts for each attribute from the ADD list or the DBNAME.DMD file. Table A.5 lists valid responses at the attribute prompt.

\section{TABLE A.5. Commands and Options in ADD Mode at the Attribute Prompts for a New Record}

Input option
or command


After the user inputs the values for each attribute in the new record per the ADD list, ALPHAMA displays the Action? prompt. At this point, the user may input one of the responses listed in Table A. 6 .

\begin{tabular}{|c|c|}
\hline $\begin{array}{l}\text { Input option } \\
\text { or command }\end{array}$ & $\begin{array}{c}\text { Description of command or option } \\
\text { and valid responses }\end{array}$ \\
\hline \multicolumn{2}{|l|}{ Action? } \\
\hline ABORT & $\begin{array}{l}\text { - Cancel ADD mode; return to the search } \\
\text { for: prompt (record is NOT SAVED) }\end{array}$ \\
\hline ADD & - Add the record; return to search for: \\
\hline DISPLAY & - Display the current record data \\
\hline EDIT & - Edit the record before adding it \\
\hline END & - Add the record; return to search for: \\
\hline$<$ Enter $>$ & - Add the record; return to search for: \\
\hline QUIT & $\begin{array}{l}\text { - Add the record; exit the ALPHAMA session } \\
\text { and retuxn to the system prompt }\end{array}$ \\
\hline
\end{tabular}



A P E N D I $X$
B.
R $\mathbf{8}$
$\nabla \mathbf{X}$
E X M P L E
S D P P O R T
A N D
A X I L I A R Y
F I I E 8

\section{B. 1 TETMA.DMD}

TSTMA.DMD (Exhibit B.I) is the data description file for the database TSTMA.DMS used in the example sessions.

\begin{tabular}{|c|c|c|c|c|c|c|}
\hline Attr. Ic ng name & & $\begin{array}{l}\text { ttr. } \\
\text { abbrev. }\end{array}$ & Type & & & $\begin{array}{l}\text { Rey } \\
\text { status }\end{array}$ \\
\hline ATTR UNIQUE UID 1 & OR & IDI & TEXT 9 & LEN & 9 & KEY \\
\hline ATTR UNIQUE_UID_2 & OR & ID2 & TEXT 7 & LEN & 7 & $\mathrm{KEY}$ \\
\hline ATTR UNIQUE=UID-3 & OR & ID3 & INTEGER & ILEN & 2 & KEY \\
\hline ATTR UNIQUE UID ${ }^{-} 4$ & OR & ID4 & INTEGER & LEN & 1 & KEY \\
\hline ATTR TEXT ND 1 & OR & TEXT1 & TEXT 14 & LEN & 14 & \\
\hline ATTR TEXT_NO-2 & OR & TEXT2 & TEXT 20 & LEN & 20 & \\
\hline ATTR TEXT' $\mathrm{NO}^{-} 3$ & OR & TEXT3 & TEXT 60 & LEN & 60 & \\
\hline ATTR TEXT'- $\mathrm{NO}^{-} 4$ & OR & TEXT4 & TEXT 600 & LEN & 600 & \\
\hline ATTR INTEGER NO 1 & OR & INT1 & INTEGER & LEN & 6 & \\
\hline ATTR REAL_NO_1 & $3 x$ & REALI & REAL & IEN & 9 & \\
\hline ATTR REAL=NO- 2 & $O R$ & REAL2 & REAL & LEN & 11 & \\
\hline ATTR ASSOCIA $\bar{T} E$ DIR CODE & OR & AD & TEXT 1 & LEN & 1 & KEY \\
\hline ATTR EXPONENTIĀL_FŌRMAT_ 1 & OR & EF1 & REAL & LEN & 11 & \\
\hline ATTR EXPONENTIAL_FORMAT_2 & OR & EF2 & REAL & LEN & 11 & \\
\hline ATTR DATE_NUMBER_1 & OR & DN1 & DATE & LEN & 10 & \\
\hline
\end{tabular}

\section{B. 2 TETMA. TBL}

TSTMA.TBL (Exhibit B.2) is the 16-row master table used for the example sessions. (To prevent screen "wrapping" here, Exhibit B.2 divides the table's 16 columns into three sections; the first column in section 1 , which is repeated here in subsequent sections for readability, would appear only once in actual output.) 


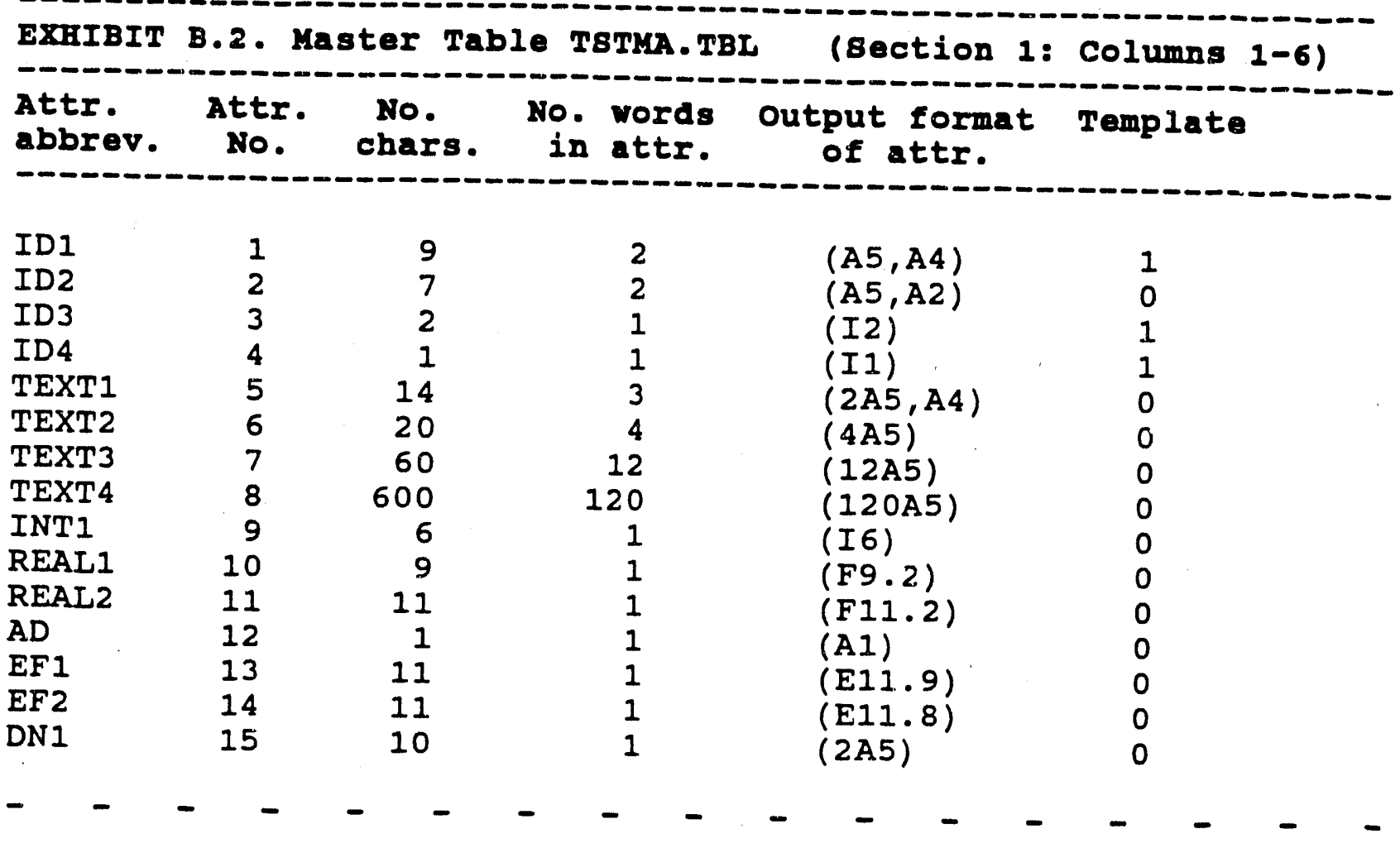

EXIIIBIT B.2. Master Table TsTMA.TBL (8ection 2: Columns 7-12)

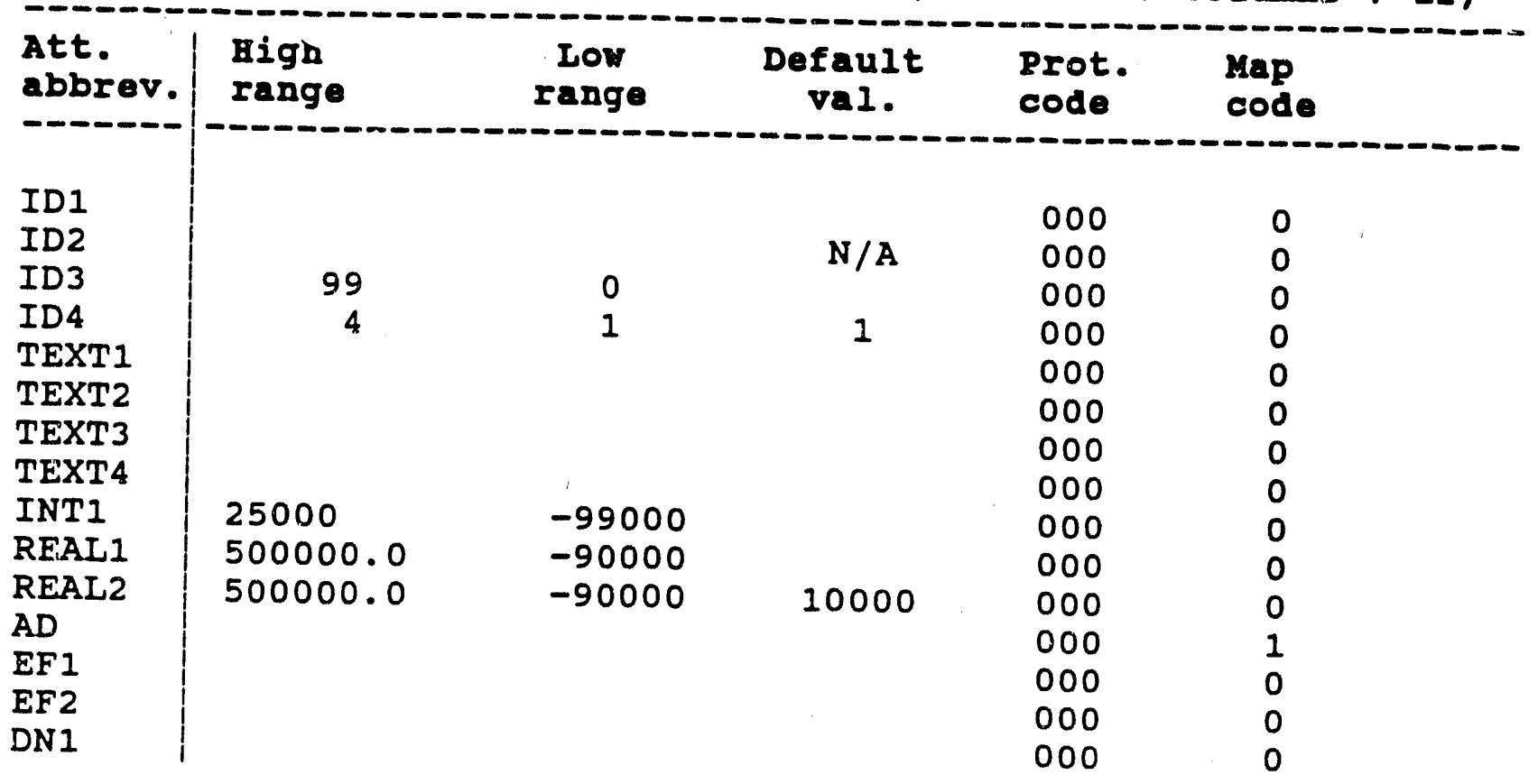




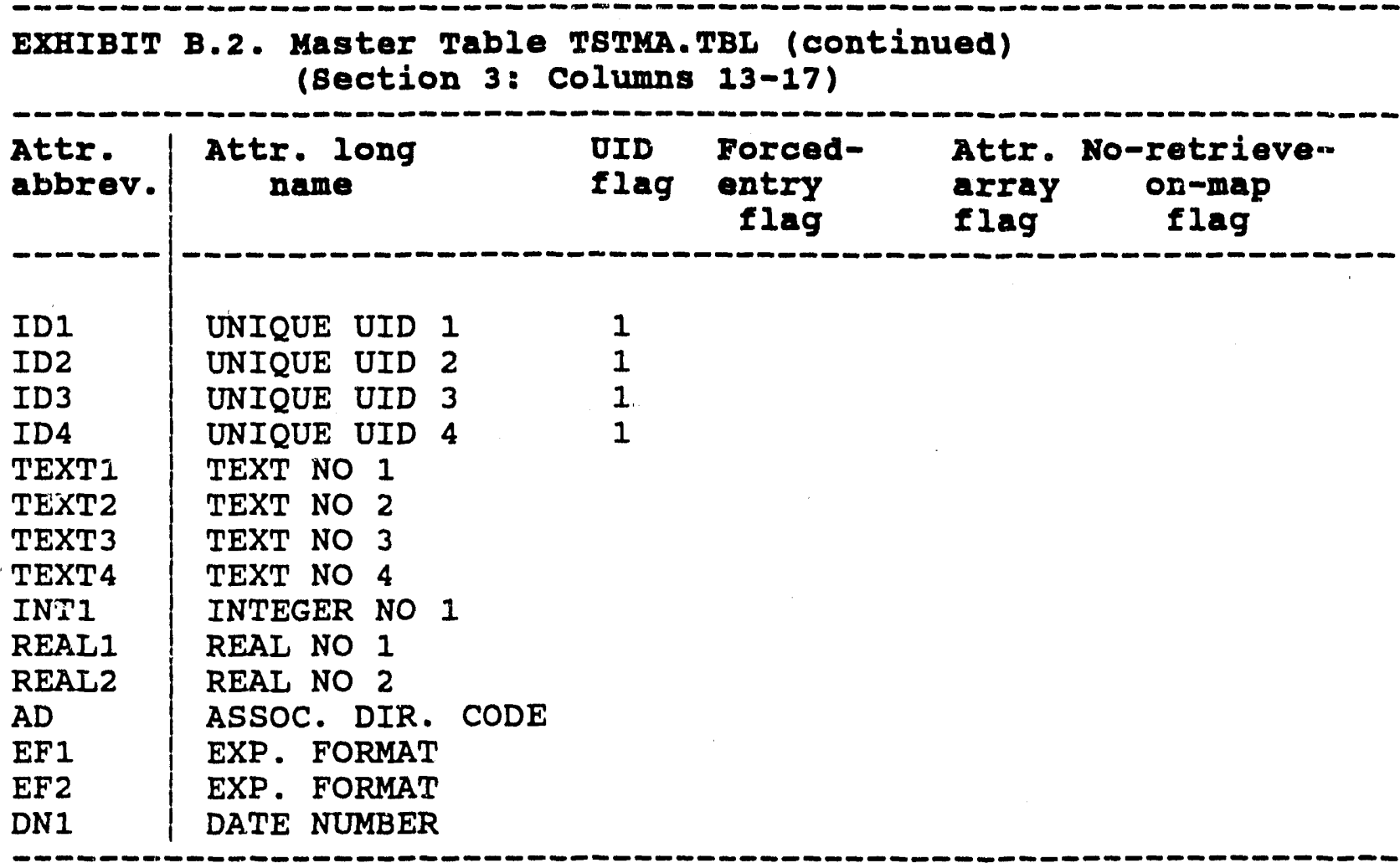

\section{B.3 TSTKA.REF}

TSTMA.REF is the reference file used for the example sessions. It identifies the support files that ALPHAMA should open for TSTMA.DMS. In this case, the database has one support file: ADNA.DMS. Thus when you access the test database that you copied to your area, TSTMA.REF directs ALPHAMA to open ADNA.DMS also.

EXHIBIT B.3. Reference File TSTMA.REF

GUIDE_DIR_GENERAL:ADNA.DMS

\section{B. 4 TSTMA.TEY}

TSTMA.TEM (Exhibit B.4) is the template file used for the example sessions. 
EXrIBIT B.4. Template File TSTMA.TEM

Attribute Template*

$\begin{array}{ll}\text { ID1 } & \\ \text { ID1 } & 2 \times 6 \mathrm{NX} \\ \text { ID3 } & 9 \mathrm{~S} \\ \text { ID4 } & 2 \mathrm{~N} \\ & \mathrm{~N}\end{array}$

1D4 $\mathrm{N}$

$\star \mathrm{X}=$ letter or number; $\mathrm{N}=$ number; $\mathrm{S}=$ space (blank).

\section{B.5 TSTMA. CHA}

TSTMA.CHA (Exhibit B.5) provides an illustration of the change log produced by the ALPHAMA program. The first list of ID1, ID2, ID3, and ID4 displays the current values of the attributes. Then after the double underscore, AIPHAMA displays the previous (old) values and the current (edited) values. (Text characters align flush left; numeric characters, flush right.)

\section{EXHIBIT B.5. Example Change Log File TSTMA.CHA}

\section{$12 / 21 / 91 \quad 14: 21 \quad[201,24710]$}

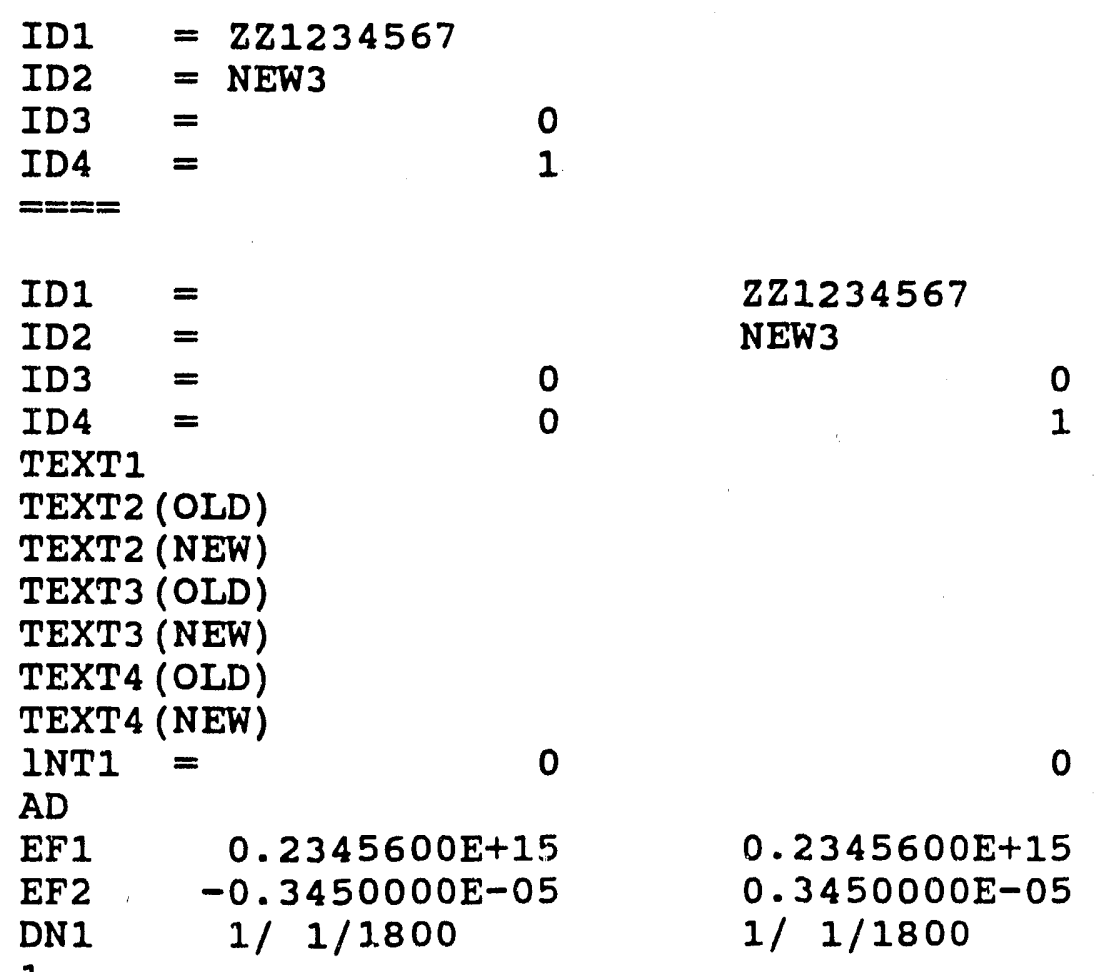

1-- - 


\section{B.6 TSTKA. ADD}

TSTMA.ADD (Exhibit B.6) is the ADD file used for the example sessions.

\section{EXHIBIT B.6. Add File TBTMA.ADD}

Attribute abtreviation:

ID1

ID2

ID3

ID4

TEXT1

TEXT2

TEXT4

INT1

REAL1

REAL2

$A D$

EFI

EF2

DN1

\section{B.7 TSTKA. SKP}

TSTMA.SKP (Exhibit B.7) is the SKIP file used for the example sessions.

\section{EXHIBIT T. Bkip File TSTMA.8KP}

Attribute abbreviation:

IDI

ID2

TEXT1

INT1

$\mathrm{AD}$

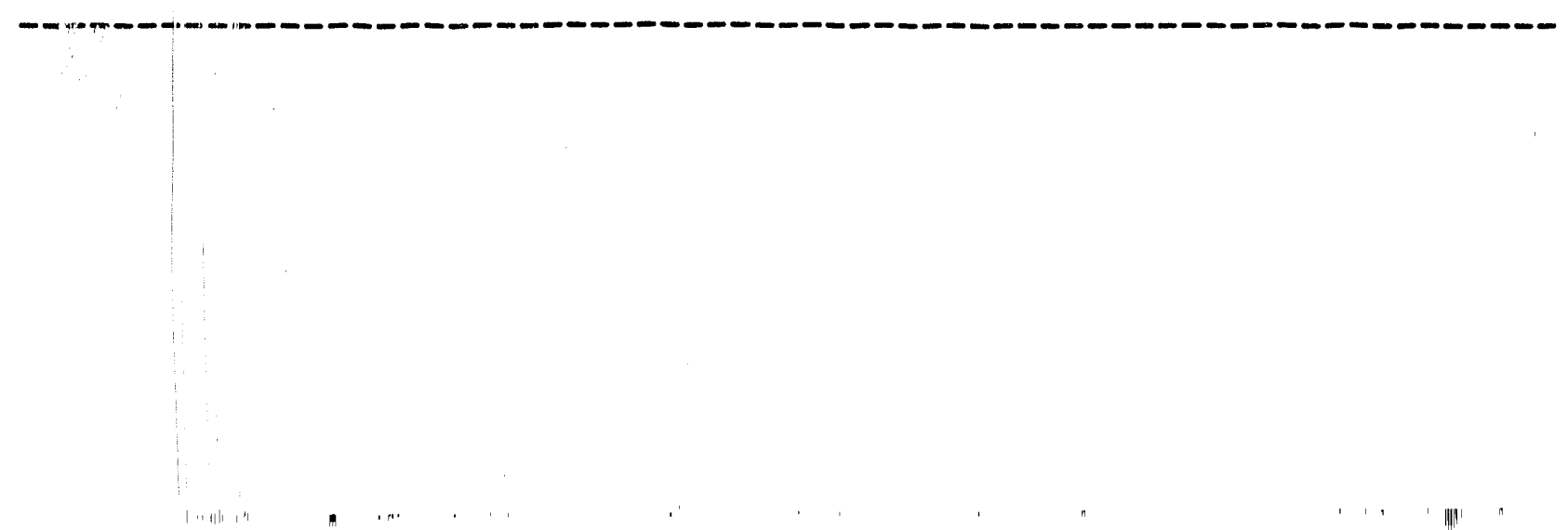




\section{B.8 TBTYA. ERR}

The following TSTMA.ERR listing (Exhibit B.8) illustrates the error file produced when input to ALPHAMA is an unresolved condition. In this case, the database master table had the template flag on, but ALPHAMA found no templates for five attributes

\section{EXHIBIT B.8. Example Error File TSTMA.ERR}

$\begin{array}{llll}\text { 12-Aug-91 } & 14: 06 & \text { WARNING : No template for attribute : PROG. } \\ \text { 12-Aug-91 } & 14: 06 & \text { WARNING : No template for attribute : SPROG. } \\ \text { 12-Aug-91 } & 14: 06 & \text { WARNING : No template for attribute : CATE } \\ \text { 12-Aug-91 } & 14: 06 & \text { WARNING : No template for attribute : TASK } \\ 12-A u g-91 & 14: 06 & \text { WARNING : No template for attribute: }\end{array}$




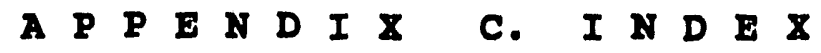

ABORT

ADD mode $3.6 .6 ; 3.6 .6 .5 ; 3.6 .4 ; 5.1$ item 29

EDIT mode 3.7 .11

SEARCH mode $3.5 ; 3.5 .1$

Abbreviations (short forms)

Attributes Exibit $9 ; 4.4$

Prompts Exhibit 1; Exhibit 3

Accessing ALPHAMA 3.1; Exhibit 2

Action? Prompt 3.6 .6

$\mathrm{ADD}$

Command 3.6 .6

Aborting ADD 3.6.6.4

ADD at Action? prompt 3.6 .6 .2

List

ADD options Table 3

File (.ADD) $3.6 ; 4.6$

With SKIP 3.6 .3

Mode 3.6

Prompt

Options Table 3

When search fails $3.5 .1 ; 3.6$

When attribute text is deleted 3.7 .2 .5

Adding Records -- See ADD

Commands 3.6

Example session 5.1

ALPHAMA 1.1

APPLY 3.7 .8 ; Exhibit 9

Attribute

Array flag 4.2 item 16; Table 5

Command 3.7 .4

Keyed Table 4

Maintenance

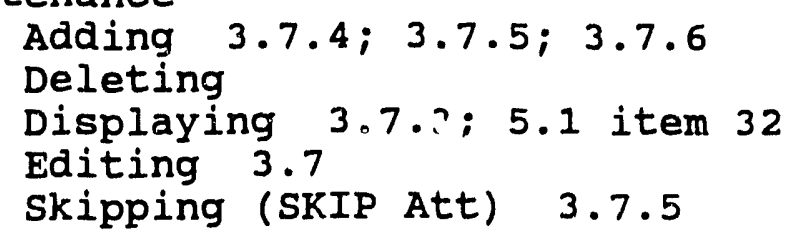




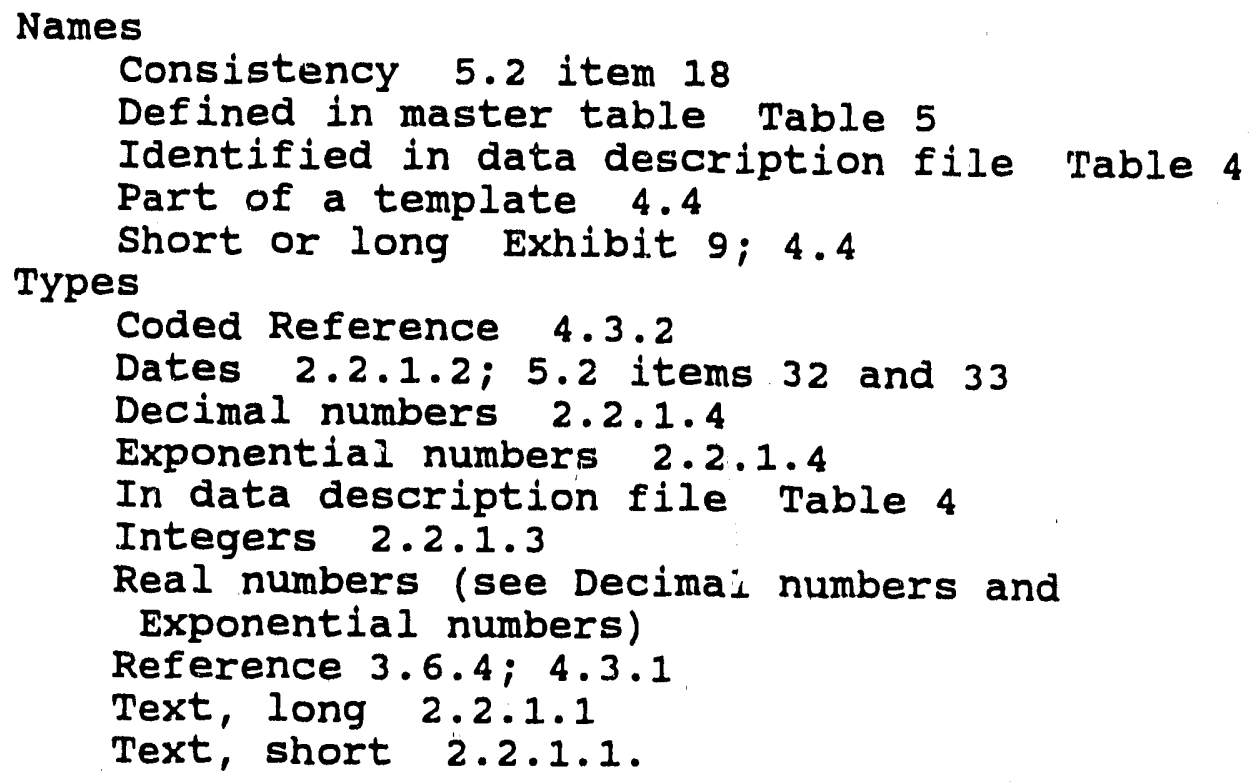

Auto Update $3.6 .4 ; 3.7 .6 ; 4.3 .1$

Auxiliary File -- See support File

Blanks

For deleted value 3.7 .2 .2

Text attribute default (ADD) 2.2 .3

Change

Change (.CHA) File 4.5

Change (open) a database 3.4

Code

Add/Update 4.2

Attribute $4.3 .2 ; 5.1$ items 26 and $31 ; 5.2$ items 16 and 34

Filecode $3.4 ; 5.1$ item 11

Map (reference) $4.2 ; 4.3 .1$

Protection code 4.2

Coded Attribute 2.5.4.2; 4.3.2; 5.1 items 26 and 31 ;

5.2 items 16 and 34

Comma 2.2.1.3; 2.2.1.5; 2.2.2

Commands

General and prompt-specific 2.1.1

Functions chap. 3

Copying Example Files 5.0; 5.1 item 7

$<$ Ctrl $>-C \quad 3.2 .2$

Custom Applications Chap. 6 


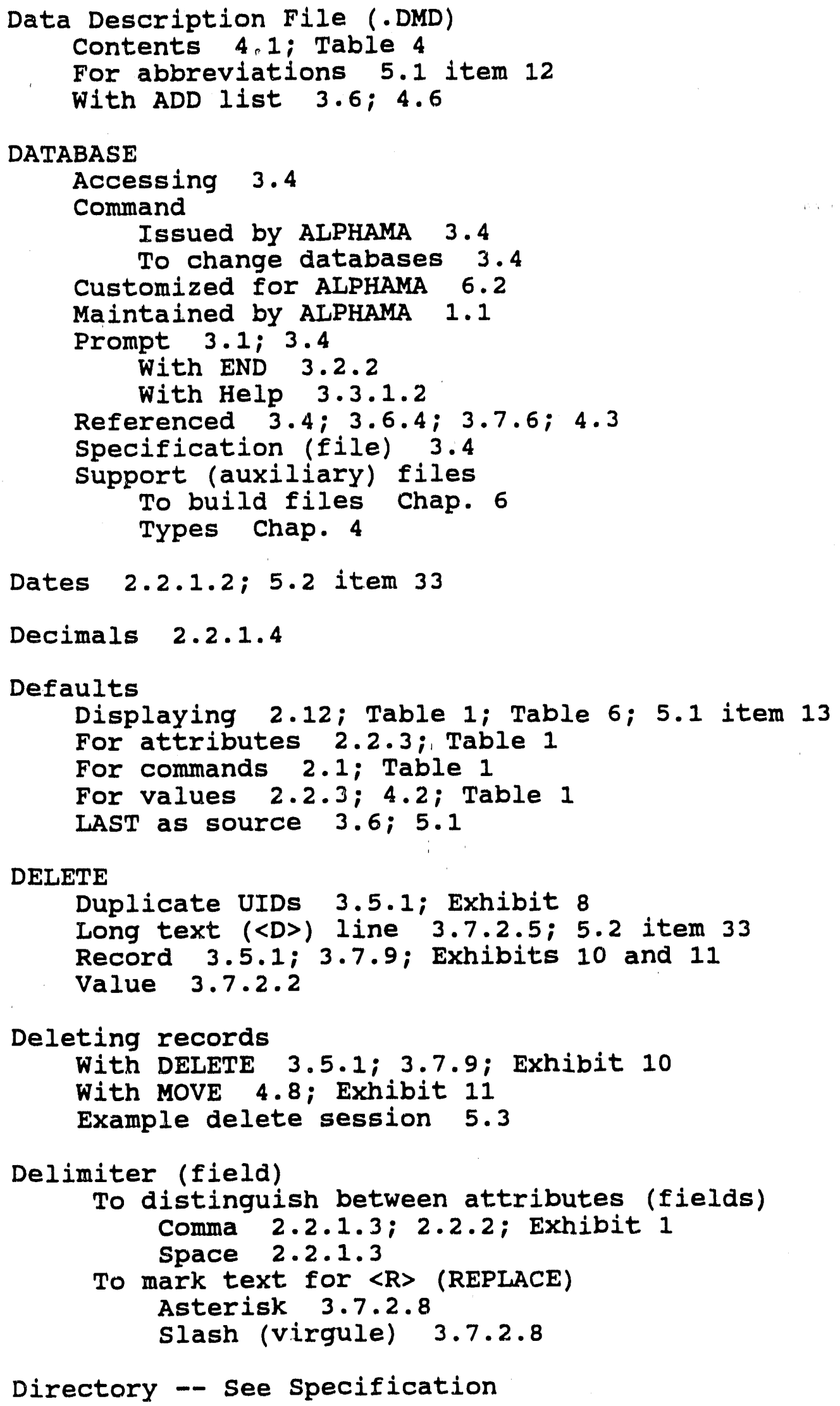

DELETE

Duplicate UIDs 3.5 .1 ; Exhibit 8

Long text $(\angle D>)$ line $3.7 .2 .5 ; 5.2$ item 33

Record $3.5 .1 ; 3.7 .9 ;$ Exhibits 10 and 11

Value 3.7 .2 .2

Deleting records

With DELETE $3.5 .1 ; 3.7 .9$; Exhibit 10

With MOVE 4.8 ; Exhibit 11

Example delete session 5.3

Delimiter (field)

To distinguish between attributes (fields)

Comma 2.2.1.3; 2.2.2; Exhibit 1

space 2.2 .1 .3

To mark text for <R> (REPLACE)

Asterisk 3.7.2.8

Slash (virgule) 3.7.2.8

Directory -- see specification 


\section{DISPLAY}

Command

$$
\begin{aligned}
& \text { ADD mode } 3.6 .6 .1 \\
& \text { EDIT mode } 3.7 .2 .3 ; 3.7 .2 .4 ; 3.7 .3
\end{aligned}
$$

Number of active records Exhibit 6

Previous line 3.7 .2 .4

Record (current) $3.6 .6 .1 ; 3.7 .3 ; 5.2$ item 16

Values

Of attribute in referenced database Table 2

of current UID 3.5.2.2

of selection set UIDs 3.5 .2 .1

Duplicate Unique Identifiers 3.5 .1 ; Exhibit 8

EDIT

Command $3 \cdot 6 \cdot 6 \cdot 3$

Mode $3.7 ; 3.7 .1$ through 3.7.13

Prompt

Indication of EDIT mode 3.74

Second-level help for Exhibit 3

Editing Records -- See EDIT

Example session 5.2

With long text attributes $3.7 .2 ; 3.7 .2 .1$ through 3.7 .2 .9

with short text attributes 3.7 .1

END

Command $3.2 .2 ; 3.6 .6 .5 ; 3.7 .12$

EDIT mode 3.7 .2 .9

<Enter> Key

Command

Types 2.1 .1

To accept

Current value 3.7

Default 2.1.2; 2.2.3; 4.2

To display text line 3.7 .2 .3

To initiate processing 2.1 .1

Accept changes to long text attribute 3.7 .2

Add record $3.6 ; 3.6 .6 ; 3.6 .6 .2$

Append text 3.7 .2 .7

Insert text 3.7 .2 .6

To skip members of array 4.2

To terminate

Change session 4.5

Editing 3.5.2.1; 3.7.2.9

Input for long text attribute

Invalid 2.2.1.1

Valid 4.4

Error

Error (.ERR) File 4.9

Messages 2.2 .1 .4 


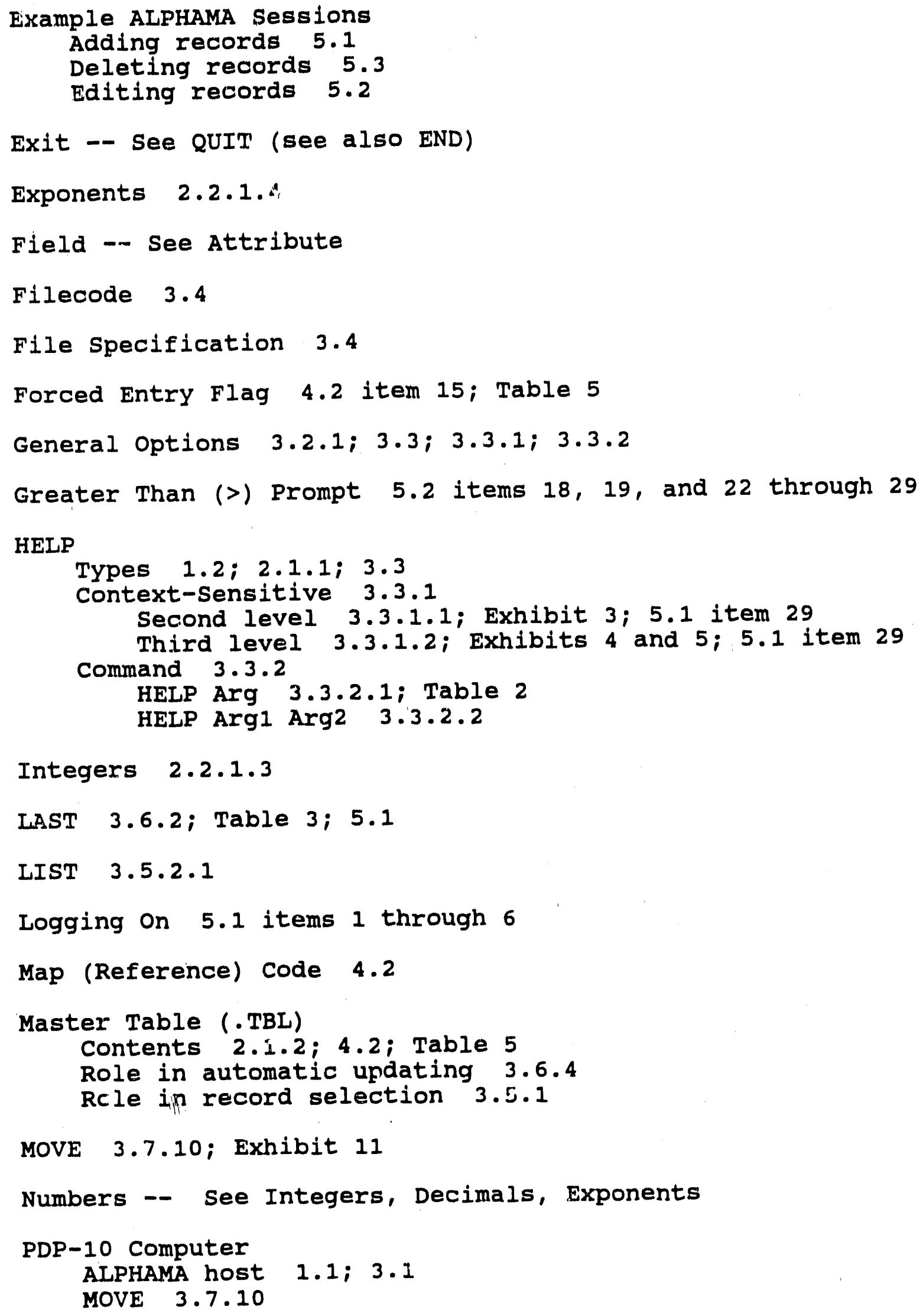


Prompts

Displayed 2.1.2; Table 1; 5.1 item 13

Typess

ALPHAMA -- See Action? Prompt, ADD, DATABASE, EDIT, Greater Than $(>)$, Search

system -- see system

Prompt-Specific Commands Chaps. 5, 6, 7

Protection Code 4.2 item 10

Question Mark(s) 3.3.1.1; 3.3.1.2

QUIT $3.2 .1 ; 3.6 .6 .6 ; 3.7 .13$

Quotation Marks 2.2.2

Ranges, Attribute Values Table 5; 4.2 items 7 and 8

Records

Displaying $3.7 .3 ; 3.7 .2 .1$

In active database Exhibit 6

Maintaining

$$
\text { Adding } 3.6 ; 3.6 .4 ; 5.1
$$

Appending 3.7 .2 .7

Deleting $3.7 .9 ; 5.3$; Exhibit 10

Editing $3.7 ; 3.7 .6 ; 5.2$

selecting (specifying) 3.5 .1

Reference

Map (reference) code 4.2 item 12 ; Table 5

Reference (.REF) File 4.3

Referenced

Attribute $3.6 .4,4.3 .1$

Attribute, coded $4.3 .2,5.1$ items 26 and 28

Data 4.3

REPLACE

String 3.7 .2 .8

Retrieve

Records -- See Search

Referenced attribute 4.2 item 17, Table 5

SAVE 3.7 .7 ; Exhibit 9

Search

For records 3.5 .1

Prompt 3.5 .1

SEARCH mode 3.5

SHOW 3.7 .2 .1 
SKIP

Command (Att) 3.7 .5

SKIP (.SKP) File 4.7

Space 2.2

specification

Attribute

With LIST 3.5.2.1

With SKIP 3.6 .6

With SKIP Att 3.7 .5

Default (values) 4.2

Format (template) 4.4

Range (values) 4.1; 4.2; Table 5

Record (UID) 3.5.1

File (Database) 3.4

Star Multiplier 3.6 .5

Stringed Input 3.6 .1

Support File

To build files chap. 6

Types Chap. 4

system

Backup for DBRECOVER 4.5

Exiting to $3.2 .1 ; 3.2 .2$

Help for 3.3.2.1; Table 2

System 1022 and 1032

Databases maintained by ALPHAMA 1.1

Dupl icate records 3.5 .1

System 1022 DFIND 3.7 .10

system (monitor) prompt

Accessing ALPHAMA 3.1

Copying files $5.0 ; 5.1$ item 7

Deleting files 5.1 item 7

System select $1.1 ; 3.1$

Template

Flag in master table 4.2 item 6 , Table 5

Template (.TEM) File 4.4

Text

Documentation files 1.2

Attributes -- See Attributes

Inputting - $3.71 ; 3.72$

UID -- See Unique Identifier(s) 
Unique Identifier(s)

Composed of attributes 3.5

Displayed for selection set 3.5 .2 .1

Set ( $f$ lag) in master table 4.2

Used to select record 3.5.1; Exhibit 7

Values (current UID) displayed 3.5.2.2

Update

Authorization 1.1

Automatic $3.6 .4 ; 3.7 .6 ; 4.3 .1$

Code 4.2 item 11 , Table 5

Value (s)

Defaults $2.2 .3 ; 4.2$; Table 1

Displaying $3.5 .2 .1 ; 3.6 .6 .1 ; 3.7 .3$

Maintaining

Automatic updating 3.7 .6

Changing with APPLY 3.7 .8

Deleting 7.3.2.2

Editing $3.6 .6 .3 ; 3.6 .4 ; 3.6 .5$

VAX Computer

ALPHAMA host $1.1 ; 3.1$ 
ALPHAMA USER'S GUIDE (V5C)

ALPHA System welcomes your comments and suggestions on the quality and usefulness of this publication. Your input is an important part of the information used for revisions.

* Is the manual useful to you?

* If so, which parts of the manual are the most useful?

* Which parts of the manual are the least useful?

* Did you find any errors?

* Is the information clearly presented?

* Do you need more information? If so, where?

* Do you need more examples? If so, where?

If you find any errors or have any other suggestions for improvements, please indicate the topic and page number below:

Please send your comments to:

ALPHA System

$\mathrm{J}$. K. Lovin

Jackson Plaza, MS-7610

Electronic mail: JKL

Phone: 6-2859

If you would like a reply, please give your name, address, and telephone number below:

THANK YOU FOR HELPING US IMPROVE OUR DOCUMENTATION. 
This page intentionally left blank. 


\section{INTERNAL DISTRIBUTION}

1. R. L. Haese

2. B. D. Heatherly

3. S. E. Hughes

4. J. S. Ishee

5-10. J. K. Lovin

11-16. S. M. Pratt

17. W. R. Ragland

18. D. W. Smith

19. C. S. Travaglini

20. K. D. Warden

21-22. Laboratory Records Department

23. Central Research Library

24. Y-12 Document Reference Section

25. ORNL Patent Section

\section{EXTERNAL DISTRIBUTTON}

26. Office of Assistant Manager for Energy Research and Development, U.S. Department of Energy Oak Ridge Field Office, P.O. Box 2001, Oak Ridge. TN 37831

27-28. Office of Scientific and Technical Information, U.S. Department of Energy Oak Ridge Field Otfice, P.O. Box 2001, Oak Ridge, TN 37831 

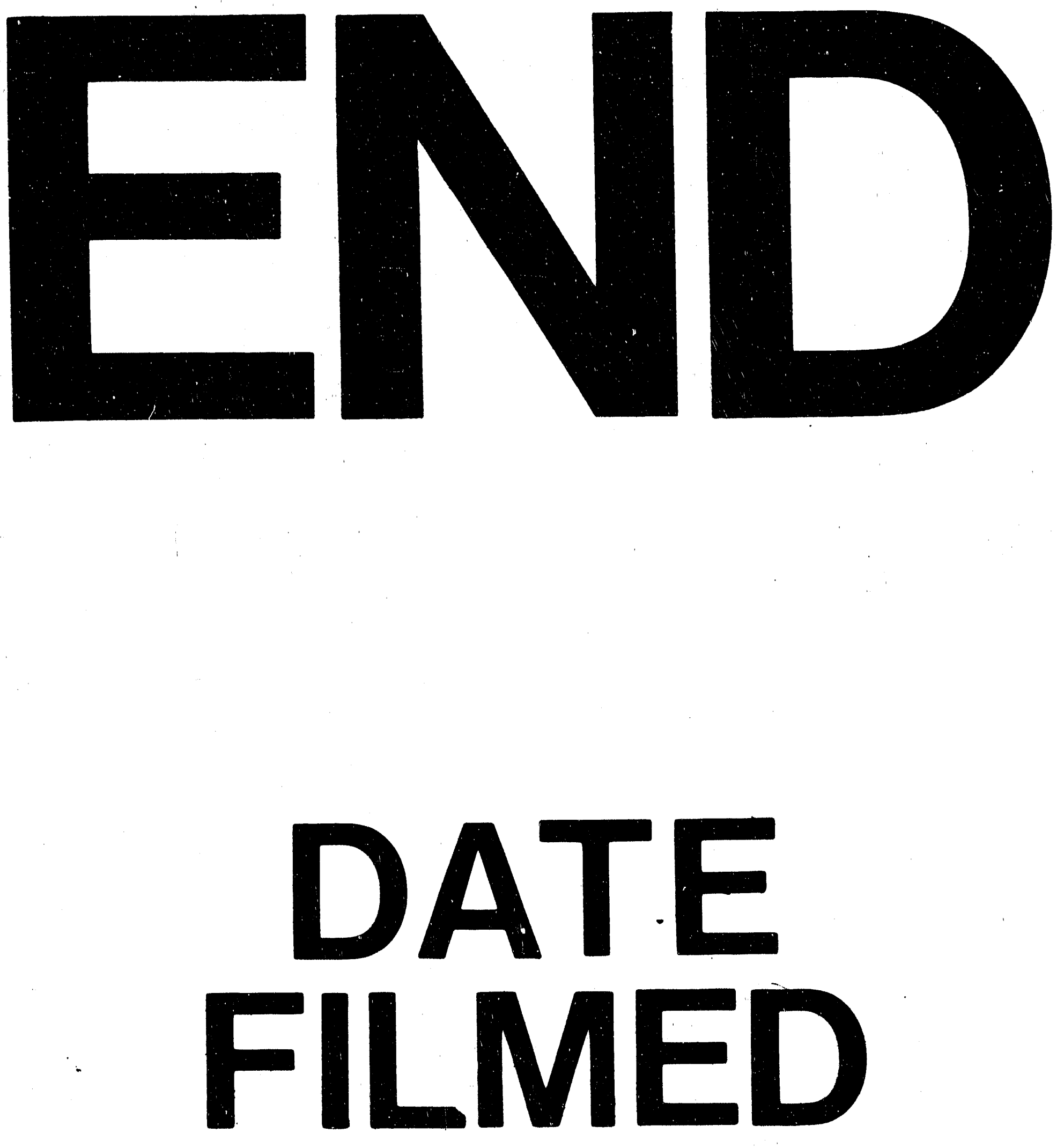

I

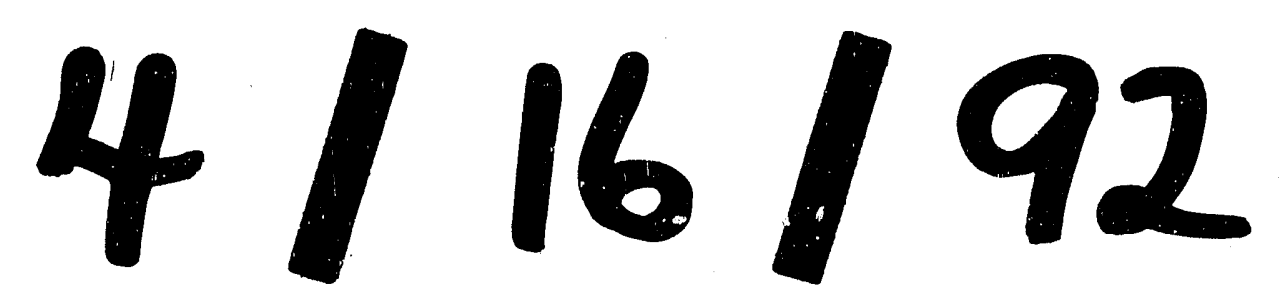


